\title{
Selected Bibliography of Statistical Literature, 1930 to 1957: IV. Markov Chains and Stochastic Processes
}

\author{
Lola S. Deming and D. Gupta*
}

(September 29, 1960)

\begin{abstract}
This is the fourth in a series of bibliographies dealing with various specific subjects in the field of statistics. Titles and references of important contributions to the study of Markov chains and stochastic processes have been taken from technical journals published since 1930 in the many countries that have been actively engaged in statistical analysis.
\end{abstract}

Two prominent reviewing journals, Zentralblatt für Mathematik (1930 to 1940) and Mathematical Reviews (1940 to 1957), have provided the source material for this series of bibliographies. The NBS Statistical Engineering Laboratory maintains a current file of abstracts cut from these journals and pasted onto cards, on the subject of probability and mathematical statistics. Each card is coded by subject after the classification system of Mathematical Reviews, and filed by author. Authors' names and references are transcribed to punched cards, and classified by subject code to facilitate use of the abstract cards. The listings therefrom, for any chosen subject, when supplied with titles, provide the manuscript for these published bibliographies. The bibliography presented here is on Markov chains and stochastic processes.

Complete coverage of any one of the subjects is, of course, impossible, but the reviewing journals have undoubtedly abstracted the most important publications from a large variety of technical journals and publishing houses. The reviewing journals have generally favored the publications in statistics that deal with theory and methodology; any subclassification on applications is consequently very limited.

Following the subject classification of the Mathematical Reviews Annual Index, all abstracts that come under the general topic of Markov chains and stochastic processes were consolidated, until 1956, into one category. Since that time, however, the Index has subdivided this general group into (A) Stochastic processes; general theory, (B) Markov processes, (C) Stationary processes, (D) Special processes, and we introduced the fifth category, (E) Applications, to include whatever publications on applications that the reviewing journals chose to abstract.

The task of subclassifying the accumulated abstracts from 1930 to 1956 into these finer groupings was accomplished at the Catholic University of America under the general supervision of Professor Eugene Lukacs. Dr. Churchill Eisenhart, Chief of

*Department of Mathematics, The Catholic University of America, Washington, D.C. the Statistical Engineering Laboratory, who originally conceived the plans for this project, continues with encouragement and assistance.

The references given here contain the following information taken directly from the abstracts:

Author: The author's surname, followed by initials only. In the case of multiple authorships, the journal reference appears with each author's name, but the title of the paper appears with the senior author only. The symbol preceding the surname denotes multiple authorship.

Title: Exactly as in the reviewing journal. Titles of separately bound publications (books, reports, theses, etc.) are in italies, followed by the publisher.

Reference to literature: Some very unconventional abbreviations were necessitated by the limited space for transcription to the punched cards. The name of the journal in italics and the number of the volume in bold face, are followed by initial page number.

Date of publication: The next figure, in parentheses, shows the date when the article or book itself appeared.

$M$ (for Mathematical Reviews) and Z (for Zentralblatt für Mathematik) are followed by the volume number and page number of the reviewing journal in which the abstract appears.

\section{A. Stochastic Processes - General Theory}

- Aczél, J., (See Jánossy) Acta Math. Acad. Sci. Hungar. 1, 209 (1950).

Adhikari, B. P., Quelques propriétés des processus stochastiques localement continus en probabilité, C.R. Acad. Sci.Paris 244, 1000 (1957) M 18, 944

-Ambrose, W., (See Doob) Ann. of Math. 41, 737 (1940).

Ambrose, W., On measurable stochastic processes, Trans. Amer. Math. Soc. 47, 66 (1940). M 1, 149

Anderson, T. W., A note on a maximum-likelihood estimate, Econometrica 15, 241 (1947). M 8, 593

- Anderson, T. W., Estimation of the parameters of a single equation in a complete system of stochastic equations, Ann. Math. Statist. 20, 46 (1949).

M 10, 464 
Arley, N., On a Dirichlet integral, Mat. Tidsskr, B 49 (1939).

M 1, 72

Arley, N., On the theory of stochastic processes and their application to the theory of cosmic radiation, Thesis, Unversity of Copenhagen (1943). M

- Arley, N., On the theory of infinite systems of differential equations and their application to the theory of stochastic processes and the perturbation theory of quantum mechanics, Acta Math. \%6, 261 (1945).

M $\boldsymbol{\%}, 161$

- Arnous, E., Sur les relations fonctionnelles vérifiées par les fonctions caractéristiques des fonctions aléatoires dérivables en movenne quadratique, C. R. Acad. Sci. Paris 226, 627 (1948). M 9, 362

- Arnous, E., Les principales familles de fonctions aléatoires et leurs propriétés, C.R.Acad. Sci. Paris 226, 785 (1948).

M 9, 598

- Arnous, E., Equations d'évolution des lois de probabilité et théorie du transfert pour les fonctions aléatoires du second ordre, C.R. Acad. Sci. Paris 226, 1127 (1948).

M 9, 599

Bachelier, L.., Probabilités des oscillations maxima, C. R. Acad. Sci. Paris 212, 836 (1941). M 3, 3 Baptist, J. H., Etude de la dépendance stochastique, Assoc. Actuair. Belges. Bull. 50, 15 (1945).

M 10, 311

Bartels, J., Wahrscheinlichkeiten und Schwankungen (Julius Springer, 1937).

$\mathrm{Z} \mathbf{1 6}, 315$

Bartlett, M. S., Recurrence and first passage times, Proc. Cambridge Philos. Soc. 49, 263 (1953).

M 14, 887

Bartlett, M. S., Processus stochastiques ponctuels, Ann. Inst. H. Poincaré 14, 35 (1954). M 16, 724

Bartlett, M. S., An introduction to stochastic processes, with special reference to methods and applications, (Cambridge University Press, 1955). M 16, 939

- Bass, J., Les équations différentielles aléatoires, C.R. Acad. Scr.Paris 221, 168 (1945). M \%, 211

- Bass, J., (See E. Arnous) C. R. Acad. Sci. Paris 226, 627 (1948).

Bass, J., Sur les propriétés des relations fonctionnelles vérifiées par les lois de probabilité des fonctions aléatoires dérivables, C.R. Acad. Sci. Paris 226, 1120 (1948).

M 9, 520

Bass, L., On the stochastic equation for the energy loss of fast electrons in matter, Proc. Indian Acad. Sci. A 54, 423 (1956).

M 18, 702

Bass, J., Sur les solutions aléatoires de certaines équations aux dérivées partielles liées à des problèmes d'hydrodynamique, J. Math. Pures Appl. 9, 33, 295 (1954).

M 16, 601

Baticle, E., Sur une loi de probabilité a priori pour l'interpretation des résultats de tirages dans une urne, C. R. Acad. Sci. Paris 228, 902 (1949).

M 10, 550

-Baxter, G., On the distribution of the supremum functional tor processes with stationary independent increments, Trans. Amer. Math. Soc. 85, 73 (1947).

M 18, 944

Bellman, R., On a generalization of classical probability theory, I. Markoff chains, Proc. Nat. Acad. Sci. U.S.A. 39, 1075 (1953).

M 15, 330
Bendat, J., A general theory of linear prediction and filtering, J. Soc. Indust. Appl. Math. 4, 131 (1956).

M 18, 342

Bernstein, S., Correction d'une démonstration, C.R. (Doklady) Acad. Sci. URSS 25, 707 (1939).

M 1, 345

Bernstein, S., Sur un problème du schéma des urnes à composition variable, C.R. (Doklady) Acad. Sci. URSS 28, 5 (1940).

M $\boldsymbol{2}, 229$

Birkhoff, G., Dependent probabilities and spaces (L), Proc. Nat. Acad. Sci. U. S. A. 24, 154 (1938).

Z 18, 264

Birkhoff, G., Lattice theory (Amer. Math. Society N. Y., 1940).

M 1, 325

Bjerke, Bj., Wahrscheinlichkeit auf Grund von Stichproben, Norsk Mat. Tidsskr. 22, 64 (1940).

Z 23, 338

Blackwell, D., An analog of the minimax theorem for vector payoffs, Pacific J. Math. 6, 1 (1956).

M 18, 450

Blanc-Lapierre, A., Remarques sur l'analyse harmonique des fonctions aléatoires, Revue Sci. 85, 1027 (1947).

M 10, 132

Blanc-Lapierre, A., Remarques sur les propriétés énergétiques des fonctions aléatoires, $C$. R. Acad. Sci. Paris 225, 982 (1947).

M 9, 293

-Blanc-Lapierre, A., Analyse harmonique des fonctions aléatoires et caractère stationnaire, $C . R$. Acad. Sci. Paris 225, 1119 (1947). $\quad$ M 9, 293

Blanc Lapierre, A., Sur quelques problèmes posés par la détermination des spectres de puissance ou d'énergie des grandeurs aléatoires, C.R. Acad. Sci. Paris 225, 1264 (1947).

M 9, 293

Blanc Lapierre, A., Considérations sur l'analyse harmonique des fonctions aléatoires, Colloq. Intern. Centre Nat. de la Rech. Sci. 13, Paris (1949).

M 11, 257

-Blanc-Lapierre, A., Remarques sur quelques propriétés de fonctions aléatoires stationnaires intervenant dans des problèmes de changement de fréquence, C. R. Acad. Sci. Paris 242, 2799 (1956).

M 18, 76

Bobrov, A. A., On determination of the order of stochastic growth of sums of random quantities, Ukrain. Mat. Žurnal 4, 393 (1952). M 15, 238

Bochner, S., Finitely additive set functions and stochastic processes, Proc. Nat. Acad. Sci. U.S.A. 32, 259 (1946).

M 8, 215

Bochner, S., Stochastic processes, Ann. of Math. 48, 1014 (1947).

M 9, 193

Bochner, S., Partical ordering in theory of stochastic processes, Proc. Nat. Acad. Sci. U.S.A. 36, 439 (1950).

M 12, 425

Bochner, S., Stochastic processes with finite and nonfinite variance, Proc. Nat. Acad. Sci. U.S.A. 39, 190 (1953).

M 14, 771

Bochner, S., Boundedness and stationarity of time series, Proc. Nat. Acad. Sci. U.S.A. 40, 289 (1954)

M 15, 807

Bochner, S., Limit theorems for homogeneous stochastic processes, Proc. Nat. Acad. Sci. U.S.A. 40, 699 (1954).

M 16, 379 
Bochner, S., Partial ordering in the theory of martingales, Ann. of Math. 62, 162 (1955).

M 1\%, 167

Bopp, F., Einfaches Beispiel aus der stochastischen Quantenmechanik, Z. Physik. 143, 233 (1955).

M 18, 259

Bopp, F., Würfel-Brettspiele, deren Steine sich näherungsweise quantenmechanisch bewegen, $Z$. Naturforschung 10a, 783 (1955).

M 18, 259

Bopp, F., Eine Art Phasenraum-Darstellung der Quantenmechanik, Z. Physik. 144, 13 (1956).

M 18, 259

Bopp, F., Quantenmechanische und stochastische Prozesse, Z. Naturforschung 10a, 789 (1956).

M 18, 259

Borchsenius, V., (See N. Arley) Acta Math. 76, 261 (1945).

Borel, E., Sur l'emploi du théorème de Bernoulli pour faciliter le calcul d'une infinité de coefficients. Application au problème de l'attente à un guichet, C. R. Acad. Sci. Paris 214, 452 (1942). M 4, 248

Breny, H., À propos de la notion de fonction aléatoire, Ann. Soc. Sci. Bruxelles \%o, 96 (1956).

M 18, 240

Brunk, H. D., On the application of the individual ergodic theorem to discrete stochastic processes, Trans. Amer. Math. Soc. 78, 482 (1955).

M 16, 724

Bruno, G. G., La legge sinusoidale delle serie derivate con somme mobili, Statistica, Milano 10, 351 (1950).

M 12, 426

- Cameron, R. H., Transtormations of Wiener integrals under translations, Ann. of Math. 45, 386 (1944).

M 6, 5

-Cameron, R. H., Evaluation of various Wiener integrals by use of certain Sturm-Liouville differential equations, Bull. Amer. Math. Soc. 51, 73 (1945).

M 6, 160

-Cameron, R. H., The behavior of measure and measurability under change of scale in Wiener space, Bull. Amer. Math. Soc. 53, 130 (1947).

M 8, 392

Campagne, C., The theorem of Hattendorf and its general validity by the theorem of Cantelli, Verzekerings-Arch. 24, 121 (1943).

M 8, 390

Cantelli, F. P., Sulle probabilità di Karup, Atti Accad. Naz. Lincei. Rend. Cl. Sci. Fis. Mat. 6, 397 (1949).

M 11, 444

-Castagnetto, L., (See F. Cernuschi) Ann. Math. Statist. 18, 122 (1947).

Čentsov, N., La convergence faible des processus stochastiques à trajectoires sans discontinuités de seconde espèce et l'approche dite "heuristique" au tests du type de Kolmogorov-Smirnov, Teor. Veroyatnost, I Primenen. 1, 155 (1956). M 18, 831 -Cernuschi, F., Probability schemes with contagion in space and time, Ann. Math. Statist. 18, 122 (1947). M 8, 472

Chung, K. L., On the renewal theorem in higher dimensions, Skand, Aktuarietidskr. 35, 188 (1953).

M 14, 994

Chung, K. L., On a stochastic approximation method, Ann. Math. Statist. 25, 463 (1954).

M 16, 272
Consael, R., Sur quelques points de la théorie des processus-stochastiques, Acad. Roy. Belgique. Bull. Cl. Sci. 36, 870 (1950).

M 12, 723

Cox, D. R., A use of complex probabilities in the theory of stochastic processes, Proc. Cambridge Philos. Soc. 51, 313 (1955).

M 16, 938

Cox, D. R., The analysis of non-Markovian stochastic processes by the inclusion of supplementary variables, Proc. Cambridge Philos. Soc. 51, 433 (1955).

M 16, 1129

Cramér, H., On the theory of stochastic processes, C. R. Cong. Math. Scand. (J. G. Forlag, Copenhagen, 1947).

M 8, 390

Cramér, H., Problems in probability theory, Ann. Math. Statist. 18, 165 (1947). M 8, 591

Cramér, H., A contribution to the theory of stochastic processes, Proc. Symp. Math. and Prob., 329, (U. Calif. Press, L.A. 1951).

M 13, 475

Darmois, G., Analyse générale des liaisons stochastiques. Etude particulière de l'analyse factorielle linéaire, Rev. Inst. Internat. Statistique $\mathbf{2 1 ,} 2$ (1953).

M 15, 809

-Dedebant, G., Sur les équations aux dérivées partielles que verifient les fonctions de distribution d'un champ aléatoire, C.R. Acad. Sci. Paris 210, 243 (1940).

M 1, 246

-Dedebant, G., Mécanique aléatoire. I. Le calcul aléatoire, Portugaliae Phys. 1, 95 (1944). M \%, 315 -Dedebant, G., (See J. Bass) C. R. Acad. Sci. Paris 221, 168 (1945).

Dedebant, G., Sur le calcul aléatoire, Anais Fac. Ci. Pôrto 32, 5 (1947).

M 10, 386

Derman, C., An application of Chung's lemma to the Kieter-Wolfowitz stochastic approximation procedure, Ann. Math. Statist. 2\%, 532 (1956).

M 1\%, 1218

Dmitriev, N., On the characteristic numbers of a stochastic matrix, C. R. Acad. Sci. URSS 49, 159 (1945).

M $\boldsymbol{\gamma}, 407$

-Dmitriev, N., On characteristic roots of stochastic matrices, Bull. Acad. Sci. URSS. Sér. Math. 10, 167 (1946).

M 8, 129

Doeblin, W., Sur l'équation de Smoluchowsky, Prakt, Akad. Athenon 12, 116 (1937). $\quad$ Z 18, 33

-Doeblin, W., Sur deux notes de MM. Kryloff et Bogoliouboff, C. R. Acad. Sci., Paris 204, 1699 (1937).

Z 1\%, 316

Doeblin, W., Sur deux problèmes de M. Kolmogoroff concernant les chaines dénombrables, Bull. Soc. Math. France 66, 210 (1938).

$\mathrm{Z} \mathbf{2 0}, 146$

Doeblin, W., Sur l'équation de Kolmogoroff, $C$. R. Acad. Sci., Paris 20\%, 705 (1938).

$\mathrm{Z} \mathbf{2 0}, 147$

Doeblin, W., Sur les sommes d'un grand nombre de variables aléatoires indépendantes, Bull. Sci. Math., 63, 23 (1939).

$\mathrm{Z} \mathbf{2 1}, 146$

Doeblin, W., Sur certains mouvements aléatoires, C. R. Acad. Sci., Paris 208, 249 (1939).

Z 20, 381

Doeblin, W., Sur l'équation matricielle $A^{t+s}=\left[A^{(t)} A^{(s)}\right]$ et ses applications au calcul des probabilités, Bull. Sci. Math. 64, 35 (1940). 
-Donsker, M. D., (See G. Baxter) Trans. Amer. Math. Soc. 85, 73 (1957).

Doob, J. L., Stochastic processes depending on a continuous parameter, Trans. Amer. Math. Soc. 42, 107 (1937).

Z 1\%, 27

Doob, J. L., Stochastic processes with an integralvalued parameter, Trans. Amer. Math. Soc. 44, 87 (1938)

Z 19, 127

Doob, J. L., The law of large numbers for continuous stochastic processes, Duke Math. J. 6, 290 (1940).

M 1, 344

-Doob, J. L., On two formulations of the theory of stochastic processes depending upon a continuous parameter, Ann. of Math. 41, 737 (1940).

M 2,108

Doob, J. L., Regularity properties of certain families of chance variables, Trans. Amer. Math. Soc. 4\%, 455 (1940).

M 1, 343

Doob, J. L., What is a stochastic process? Amer. Math. Monthly 49, 648 (1942). M 4, 103

Doob, J. L., Probability in function space, Bull. Amer. Math. Soc. 53, 15 (1947).

M 8, 472

Doob, J. L., The measure-theoretic setting of probability theory, Ann. Soc. Polon. Math. 25, 199 (1952).

M 15, 447

Doob, J. L., Stochastic processes (John Wiley, N.Y., 1953).

M 15, 445

Doob, J. L., Present state and future prospects of stochastic process theory, Proc. Cong. Math., 111, 348 (Noordhoff, Groningen, 1956).

M 18, 943

Doss, S., Sur la moyenne d'un élément aléatoire abstrait, C. R. Acad. Sci. Paris 226, 1418 (1948).

M 9, 520

Doss, S., Sur la movenne d'un élément aléatoire dans

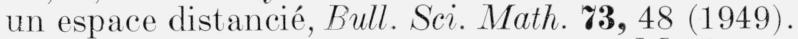

M 11, 190

Doubrowsky, V., Sur en problème limite de la théorie des probabilitiés, Bull. Acad. Sci. URSS. Sér. Math. 4, 411 (1940).

M 3, 3

Dubrovski, W., Eine Verallgemeinerung der Theorie der rein unstetigen stochastischen Prozesse von W. Feller, C. R. Acad. Sci. URSS, 19, 439 (1938).

Z 19, 73

Dugué, D., Deux notions utiles en statistique mathématique: les ensembles aléatoires bornés "en loi" et la continuité fortement uniforme en probabilité, Coll. l' Analyse Statist., Brux., p. 133 (G. Thone, Liege 1955).

M 1\%, 635

Dugué, D., Sur le second théorème limite du calcul des probabilités, C. R. Acad. Sci. Paris, 242, 444 (1956).

M 1\%,635

Dumas, M., Interprétation de résultats de tirages exhaustifs, C. R. Acad. Sci. Paris, 228, 904.

M 10, 550

$\checkmark$ Dumontet, P., (See A. Blanc-Lapierre) C. R. Acad. Sci. Paris 242, 2799 (1956).

- Dunford, N., Linear operations among summable functions, Proc. Nat. Acad. Sci. U. S. A. 25, 544 (1939).

M 1, 57

- Dunford, N., Linear operations on summable functions, Trans. Amer. Math. Soc. 4\%, 323 (1940).

M 1, 338
Dvoretzky, A., On stochastic approximation, Proc. Sympo. Stati Prob., 1, 39 (Univ. Calif. Press, Berkeley, 1956).

$\checkmark$ Dynkin, E., (See N. Dmitriev), C. R. Acad. Sci. URSS 49, 159 (1945).

Dynkin, E., (See N. Dmitriev), Bull. Acad. Sci. URSS. Sér. Math. 10, 167 (1946).

$\checkmark$ Edwards, D. A., Stochastic differential equations, Proc. Cambridge Philos. Soc. 51, 663 (1955).

M 1\%, 276

Fage, M. K., On symmetrizable matrices, Uspehi Matem. Nauk 6, 153 (1951).

M 13, 200

Fan, K., Les fonctions asymptotiquement presquepériodiques d'une variable entière et leur application à l'étude de l'itération des transformations continues, Math. Z. 48, 685 (1943). M 5, 99

Fan, K., Une définition descriptive de l'intégrale stochastique, C. R. Acad. Sci. Paris, 218, 953 (1944)

М $\boldsymbol{\gamma}, 313$

Feller, W., On the time distribution of so-called random events, Phys. Rev. 5\%, 906 (1940). M 1,344

Fisz, M., The realizations of some purely discontinuous stochastic processes, Bull. Acad. Polon. Sci. Cl. 111, 4 (1956).

M 1\%, 1097

Fisz, M., Realizations of some stochastic processes, Studia Math. 15, 359 (1956).

M 18, 519

Fomin, S., Finite invariant measures in the flows, Rec. Math. 12, 99 (1943).

M 5, 101

Fomin, S. V., On measures invariant under certain groups of transformations, Izvestiya Akad. Nauk SSSR. Ser. Mat. 14, 261 (1950).

M 12, 33

Fortet, R., Sur des probabilités en chaînes, $C . R$. Acad. Sci., Paris 204, 315 (1937).

Z 15, 406

Fortet, R., (See W. Doeblin), C. R. Acad. Sci., Paris 204, 1699 (1937).

Z 1\%, 316

Fortet, R., Sur une suite également répartie, Studia Math. 9, 54 (1940).

M 3, 169

Fortet, R., Sur la notion de fonction aléatoire, Revue Sci. 99, 135 (1941).

M \%, 19

Fortet, R., Sur la résolution des équations paraboliques linéaires, C. R. Acad. Sci., Paris 213, 553 (1941).

M 5, 123

-Fortet, R., (See Blanc-Lapierre), C. R. Acad. Sci. Paris 2\%5, 1119 (1947).

Fortet, R., Random determinants, J. Research Nat. Bur. Standards 4\%, 465 (1951).

M 13, 852

Fouillade, A., Sur l'itération de certaines substitutions linéaires, Bull. Acad. Roy. Belg., 19, 415 (1933).

Z 8, 120

Fouillade, A., Recherches sur l'iteration des substitutions fonctionnelles linéaires, Mém. Soc. Roy. Sci. Liége, IV. S. 2, 159 (1937).

$\mathrm{Z} \mathrm{19,} 67$

Fréchet, M., Sur quelques idées modernes dans la théorie des probabilités, Acta Univ. Asiae Mediae, Ser. V.-a. Easc. 32, (1940).

M 8, 472

Fréchet, M., L'intégrale abstraite d'une fonction abstraite d'une variable abstraite et son application a la moyenne d'un élément aléatoire de nature quelconque, Revue Sci. 82, 483 (1944). M 8, 141

Fréchet, M., Nouvelles définitions de la valeur moyenne et des valeurs équiprobables d'un nombre aléatoire, Ann. Univ. Lyon. Sect. A. 9, 5 (1946). 
Fréchet, M., Les espaces abstraits et leur utilité en statistique théorique et même en statistique appliquée, J. Soc. Statist. Paris 88, 410 (1947).

M 10, 386

Fréchet, M., On two new chapters in the theory of probability, Math. Mag. 22, 1 (1948). M 10, 386

Fréchet, M., Positions typiques d'un élément aléatoire de nature-quelconque, Ann. Sci. É Ecole Norm. Sup. 65, 211 (1948). M 10, 386

Fréchet, M., Sur une nouvelle définition des positions typiques d'un élément aléatoire abstrait, $C . R$. Acad. Sci., Paris 226, 1419 (1948). $\quad$ M 9, 520

Fréchet, M., Les valeurs typiques d'ordre nul ou infini d'un nombre aléatoire et leur généralisation, Colloq. Internat. Centre Nat. Rech. Sci. Paris, 13, 47 (1949).

M 11, 376

Geiringer, H., Sur les variables aléatoires arbitrairement liées, Rev. Math. Union Interbalkan. 2, 1 (1938).

Z 18, 264

Getoor, R. K., The shift operator for non-stationary stochastic processes, Duke Math. J. 23, 175 (1956).

M 1\%, 636

Ghurye, S. G., Random functions satisfying certain linear relations, Ann. Math. Statist. 25, 543 (1954).

M 16, 150

Ghurye, S. G., Random functions satisfying certain linear relations. II, Ann. Math. Statist. 26, 105 (1955)

M 16, 723

Giesekus, H., Morphologisch homogene Funktionen une ihre Erzeugung durch statistische-Superposition von Elementfunktionen. I. Allgemeine Theorie, Z. Angew. Math. Mech. 30, 154 (1950).

M 14, 296

Giesekus, H., Morphologisch homogene Funktionen und ihre Erzeugung durch statistiche Superposition von Elementfunktionen. II. Einege Hinweise und Beispiele zur Anwendung der Theorie, Z. Angew. Math. Mech. 30, 215 (1950). M 14, 296

Gihman, I. I., On the theory of differential equations of stochastic processes, Amer. Math. Soc. Transl. 1, 111 (1955).

M 1\%, 502

Gihman, I. I., On the theory of differential equations of random processes, Ukrain. Mat. Žurnal 2,37 (1950).

M 14, 61

Gihman, I. I., On some differential equations with random functions, Ukrain. Mat. Žurnal 2, 45 (1950).

M 14, 61

Gihman, I. I., On the theory of differential equations of random processes, Ukrain. Mat. Žurnal 3, 317 (1951).

M 14, 1101

Gillis, J., Correlated random walk, Proc. Cambridge Philos. Soc. 51, 639 (1955).

M 1\%, 275

Ginsbourg, G., Sur les lois limites des distributions dans les procédés stochastiques, Comm. Inst. Sci. Math. Méc. Univ. Kharkoff 17, 65 (1940).

M 3, 4

Ginsbourg, G., Sur les conditions suffisantes pour l'unicité des distributions limites, C.R. (Doklady) Acad. Sci. URSS 30, 295 (1941).

M 3, 4

Gnedenko, B., Sur la croissance des processus stochastiques homogènes à accroissements indépen- dants, Bull. Acad. Sci. URSS Sér. Math. \%, 89 (1943).

M 5, 125

Gnedenko, B. V., On the growth of homogeneous random processes with independent single-type increments, C. R. (Doklady) Acad. Sci. URSS 40, 90 (1943).

M 6, 160

Gnedenko, B. V., On the theory of growth of homogeneous random processes with independent increments, Akad. Nauk Ukrain. RSR. Zbirnik Prac' Inst. Mat. 48, 60 (1948).

M 12, 620

Goddard, L. S., Transition matrices occurring in the theory of Markoff processes, Proc. Cambridge Philos. Soc. 51, 382 (1955).

M 1\%, 936

Grenander, U., Stochastic processes and integral equations, Ark. Mat. 1, 67 (1949). M 11, 376

Haba, J., Sur les probabilités des évènements dépendants, Publ. Fac. Sci. Univ. Charles Prague 154, 21 (1937).
Z 18, 265

Hammersley, J. M., The area enclosed by Pólya's walk, Proc. Cambridge Philos. Soc. 52, 78 (1956).

M 17, 865

- Hartman, P., Asymptotic distributions and the ergodic theorem, Amer. J. Math. 61, 977 (1939).

M 1, 59

- Hartman, P., On the infinitesimal generators of integral convolutions, Amer. J. Math. 64, 273 (1942).

M 4, 18

Hess, F. G., Alternative solution to the Ehrenfest problem, Amer. Math. Monthly 61, 323 (1954).

M 15, 805

Hille, E., Functional analysis and semi-groups, (Amer. Math. Soc. Colloq. Publ. 31, N. Y., 1948).

M 9, 594

Hille, E., On the integration problem for FokkerPlanck's equation in the theory of stochastic processes, Skandinaviske Mate Kongress, Trondheim, p. 183 (J. G. T. Forlag, Oslo 1952).

M 14, 758

- Hodges, J. L., Jr., Two approximations to the Robbins-Monro process, Proc. Symp. Stat. Prob. 1, 95 (Univ. Calif. Press, Berkeley, 1956).

M 18, 947

Hopf, E., Ergodic theory, Uspehi Matem. Nauk 4, 113 (1949).

M 10, 549

Hostinsky, B., Sur la solution de l'équation généralisée de Chapman, Cas. Mat. Fys. 68, 8 (1939).

Z 21, 135

Hostinsky, B., Sur le calcul des probabilités relatives à l'évolution d'un système, Aktuárské $V \breve{d} d y \mathbf{8 , 6 1}$ (1949).

M 11, 257

Iaglom, A. M., Effective solution of linear approximation problems for processes with random, stationary $\mathrm{n}$-th increments (Translation by $M$. D. Friedman, 572 Calif. St., Newtonville, Mass., 1956).

M 18, 157

Itô, H., Principle of the minimum entropy in information theory, Proc. Japan Acad. 29, 194 (1953).

M 15, 330

Itô, K., On stochastic processes. I. Infinitely divisible laws of probability, Jap. J. Math. 18, 261 (1942).

M $\%, 312$ 
Itô, K., A screw line in Hilbert space and its application to the probability theory, Proc. Imp. Acad. Tokyo 20, 203 (1944).

M $\%, 252$

Itô, K., Stochastic integral, Proc. Imp. Acad. Tokyo 20, 519 (1944).

M \%, 313

Itô, K., On the stochastic integral, Sûgaku 1, 172 (1948)

M 14, 1101

Itô, K., Stochastic differential equations in a differentiable manifold, Nagoya Math. J. 1, 35 (1950).

M 12, 425

Itô, K., On a formula concerning stochastic differentials, Nagoya Math. J. 3, 55 (1951). M 13, 363

Itô, K., Multiple Wiener integral, J. Math. Soc. Japan 3, 157 (1951).

M 13, 364

Itô, K., Theory of Probability (Iwanami Shôten, Tokyo, 1953).

M 15, 448

Itô, K., Complex multiple Wiener integral, Jap. J. Math. 22, 63 (1953).

M 16, 151

Itô, K., Stochastic differential equations in a differentiable manifold. II, Mem. Coll. Sci. Univ. Kyoto Ser A Math 28, 81 (1953).

M 15, 636

Itô, K., Spectral type of the shift transformation of differential processes with stationary increments, Trans. Amer. Math. Soc. 81, 253 (1956).

M 18, 980

-Jánossy, L., On composed Poisson distributions. I, Acta Math. Acad. Sci. Hungar. 1, 209 (1950).

M 13, 663

Jánossy, L., A generalization of the Laplace transform in probability theory, Magyar Tud. Akad. Mat. Fiz. Oszt. Közleményei 1, 343 (1951).

M 13, 957

Kac, M., On the distribution of values of sums of the type $\Sigma f\left(2^{k} t\right)$, Ann. of Math. 47, 33 (1946).

M $\boldsymbol{y}, 436$

Kac, M., On some connections between probability theory and differential and integral equations, Proc. Symp. Math. Prob., p. 189 (Univ. Calif. Press, Berkeley, 1951).

M 13, 568

Kakutani, S., Mean ergodic theorem in abstract (L)-spaces, Proc. Imp. Acad., Tokyo 15, 121 (1939).

M 1, 59

Kallianpur, G., Intégrale de Stieltjes stochastique et un théoréme sur les fonctions aléatoires d'ensembles, C. R. Acad. Sci. Paris 232, 922 (1951).

M 13, 475

Kampé de Fériet, J., Sur une représentation des fonctions aléatoires, C. R. Acad. Sci. Paris 225, 37 (1947).

M 9, 46

Kampé de Fériet, J., Autocorrélation et spectre quadratique d'une fonction définie sur un groupe abélien localement compact, $C$. R. Acad. Sci. Paris 236, 2198 (1953).

M 14, 995

Kampé de Fériet, J., Fonctions aléatoires harmoniques dans un demi-plan, C.R. Acad Sci. Paris 23\%, 1632 (1953).

M 15, 449

Kaplan, E. L., Transformations of stationary random sequences, Math. Scand. 3, 127 (1955).

M 1\%, 275
Karhunen, K., Zur Spektraltheorie stochastischer Prozesse, Ann. Acad. Sci. Fennicae. Ser. A. I. Math. Phys. No. 34, (1946).

M 9, 292

Karhunen, K., Úber lineare Methoden in der Wahrscheinlichkeitsrechnung, Ann. Acad. Sci. Fennicae. Ser. A. I. Math. Phys. No. 37, (1947).

M 9, 292

Karhunen, K., Linear methods in the calculus of probabilities, Trabajos Estadistica 3, 59 (1952).

M 14, 387

Karhunen, K., Über ein Extrapolationsproblem in dem. Hilbertschen Raum., Skandinav. Matemat. Kong. Pp. 35-41 (J. G. T. Forlag, Oslo, 1952).

M 14, 663

Karlin, S., Representation of a class of stochastic processes, Proc. Nat. Acad. Sci. U.S.A. 41, 387 (1955).

M 1\%, 166

Kawada, Y., On the measurable stochastic process, Proc. Phys. Math. Soc. Japan 23, 512 (1941).

M 3, 170

- Kemeny, J. G., Introduction to finite mathematics (Prentice-Hall, Englewood Cliffs, N.J., 1957).

M 18, 860

Khintchine, A., Zur Theorie der unbeschränkt teilbaren Verteilungsgesetze, Rec. Math. Moscou. 2 , 79 (1937).

$\mathrm{Z} \mathbf{1 6}, 410$

Khintchine, A., Zwei Sätze über stochastische Prozesse mit stabilen Verteilungen, Rec. Math. Moscou, 3, 577 (1938).

Z 19, 318

Khintchine, A. Sur la croissance locale des processus stochastiques homogènes à accroissements indépendants, Bull. Acad. Sci. URSS, Sér, Math. 1939, 487 (1939).

$\mathrm{Z} \mathbf{2 4}, 265$

Khintchine, A., Sur la croissance locale des processus stochastiques homogènes à accroissements indépendants, Bull. Acad. Sci. URSS, Sér. Math. 1939, 487 (1939).

M 1, 344

-Kiefer, J., On the characteristics of the general queueing process, with applications to random walk, Ann. Math. Statist. 2\%, 147 (1956).

M 1\%, 980

Kimme, E. G., On the convergence of sequences of stochastic processes, Trans Amer. Math. Soc. 84, 208 (1957).

M 18, 770

Koeppler, H., Die Anwendung der Theorie der Elementarwahrscheinlichkeit zweier Abweichungen auf die Darstellung der Differentialgleichung der Frequenzfunktion zweir Variablen, Aktuár, Vèdy $\boldsymbol{\%}, 72$ (1938).

Z 18, 266

Koopman, B. O., A probabilistic generalization of matric Banach algebras, Proc. Amer. Math. Soc. 2, 404 (1951).

M 13, 473

Kozakiewicz, W., Sur les conditions nécessaires et suffisantes pour la convergence stochastique, Fundam. Math. 31, 160 (1938).

Z 19, 228

Kozulyaev, P. A., On the theory of extrapolation of stationary sequences, Moskov. Gos. Univ. Uc. Zap. 145, Mat. 3, 59 (1949).

M 1\%, 980

Krylov, N., Relaxation processes in statistical systems, Nature 153, 709 (1944).

M 6, 168

Kuznecov, P. I., On the duration of excursions of a random function, Akad. Nauk SSSR. Žurnal Tehn. Fiz. 24, 103 (1954). 
-Kuznecov, P. I., On the mathematical theory of correlated random points, Izv. Akad. Nauk SSSR. Ser. Mat. 20, 167 (1956).

M 1\%, 1218

Leader, S., Convergence topologies for measures and the existence of transition probabilities, Pacific J. Math. 6, 479 (1956).

M 18, 470

Le Cam, L., Un instrument d'étude des fonctions aléatoires: la fonctionnelle caractéristique, $C . R$. Acad. Sci. Paris 224, 710 (1947). M 8, 472

Lehmann, E. L., (See Hodges, J. L.) Proc. Symp. Stat. Prob., 1, 95 (Univ. Calif. Press, Berkeley, 1956).

Levert, C., La durée de retour et la durée d'absence de la plus grande valeur, C. R. Acad. Sci. Paris 23\%, 374 (1953).

M 15, 330

Lévy, P., Sur les intégrales dont les éléments sont des variables aléatoires indépendantes, Ann. Scuola Norm. Super. Pisa, 11, 337 (1934).

$\mathrm{Z} \mathrm{10,}, 70$

Lévy, P., Observation sur un précédent mémoire de l'auteur, Ann. Scuola Norm. Super. Pisa, 11, 217 (1935).

$\mathrm{Z} \mathrm{11,} 125$

Lévy, P., Sur certains processus stochastiques homo-

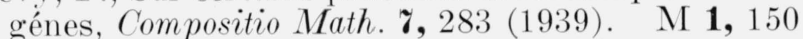

Lévy, P., Sur les discontinuités de certains processus stochastiques, C.R. Soc. Math. France Année 1938, $39(1939)$.

$\mathrm{Z} \mathbf{2 4}, 264$

Lévv, P., Intégrales stochastiques, C. R. Acad. Sci. Paris 212, 1066 (1941).

M 5, 126

Lévy, P., Dérivation, intégration et équations différentielles stochastiques, C.R.Acad.Sci. Paris 219, 602 (1944).

M $\boldsymbol{\%}, 313$

Lévv, P., Nouvelles généralisations de l'intégrale de Stieltjes, C. R. Acad. Sci. Paris 229, 644 (1949).

M 11, 166

Lévy, P., Wiener's random function, and other Laplacian random functions, Proc. Symp. Math. Prob., p. 171 (Univ. Cal. Press, Berkeley, 1951).

M 13,476

Lévy, P., Random functions: General theory with special reference to Laplacian random functions, Univ. California Publ. Statist. 1, 331 (1953).

M 14, 1099

Lévy, P., Propriétés asymptotiques de la courbe du mouvement brownien à $N$ dimensions, $C . R$. Acad. Sci. Paris 241, (1955).

M 1\%, 275

Loève, M., La loi forte des grands nombres pour des variables aléatoires liées, C.R. Acad. Sci. Paris 212, 1121 (1941).

M 5, 207

Loève, M., La tendance centrale des sommes de variables aléatoires liées, C.R. Acad. Sci. Paris 213, 9 (1941).

M 5, 207

Loève, M., Sur les fonctions aléatoires stationnaires de second ordre, Revue Sci. 83, 297 (1945).

M 8, 38

Loève, M., Fonctions aléatoires de second ordre, Revue Sci. 84, 195 (1946).

M 8, 281

Loève, M., Indicateurs abstraits et champs stochastiques, C. R. Acad. Sci. Paris 228, 1561 (1949).

M 10, 720

Lopuszanski, J., Relativisierung der Theorie der stochastischen Prozesse, Acta Phys. Polonica 12, 87 (1953).

M 15, 238
Lukacs, E., On strongly continuous stochastic processes, Sankhyā 13, 219 (1954).

M 15, 806

Lurquin, C., Sur la liaison des variables éventuelles, Mathesis 53, 8 (1939).

$\mathrm{Z}$ 20, 242

-Mabboux-Tariel, G., Spectre énérgetique de certaines fonctions aléatoires de la forme $\pm 1, C . R$. Acad. Sci. Paris 243, 1509 (1956). $\quad \vec{M}$ 18, 519 Machado, E. A., On the random function $X(t)=\int_{t_{0}}^{t} K(t, s) d \xi(s)$, Univ. Nac. Eva. Peron Publ. Esp. 48, No. 211 (1954).

M 18, 166

Mann, H. B., On the realization of stochastic processes by probability distributions in function spaces, Sankhyā 11, 3 (1951).

M 13, 141

Mann, H. B., The estimation of parameters in certain stochastic processes, Sankhyā 11, 97 (1951).

M 13, 667

Mann, H. B., Introduction to the theory of stochastic processes depending on a continuous parameter, Nat. Bur. Stds. Appl. Math. Series, 24, (U.S. G.P.O., D.C., 1953).

M 14, 663

- Martin, W. T., (See R. H. Cameron) Ann. of Math. 45, 386 (1944).

- Martin, W. T., (See R. H. Cameron) Bull. Amer. Math. Soc. 51, 73 (1945).

- Martin, W. T., (See R. H. Cameron) Bull. Amer. Math. Soc. 53, 130 (1947).

-Massignon, D., (See E. Arnous) C. R. Acad. Sci. Paris 226, 627 (1948).

- Massignon, D., (See E. Arnous) C. R. Acad. Sci. Paris 226, 785 (1948).

- Massignon, D., (See E. Arnous) C. R. Acad. Sci. Paris 226, 1127 (1948).

- Mathews, P. M., Ordinary linear differential equations involving random functions, Proc. Indian Acad. Sci. Sect. A. 43, 4 (1956).

M 18, 865

Mattila, S., On the weak topology in the theory of probability, Soc. Sci. Fenn. Comment. Phys. Math. 15, No. 14, (1950).

M 12, 619

- McGregor, J., (See Karlin, S.) Proc. Nat. Acad. Sci. U.S.A. 41, 387 (1955).

Moran, P. A. P., Some theorems on time series. II. The significance of the serial correlation coefficient, Biometrika 35, 255 (1948). M 10, 385

Moran, P. A. P., Notes on continuous stochastic phenomena, Biometrika 3\%, 17 (1950). M 12, 35

Mourier, E., Sur l'espérance mathématique d'un élément aléatoire dans un espace de Banach, C. R. Acad. Sci. Paris 299, 1300 (1949).

M 11, 376

Mourier, E., Eléments aléatoires dans un espace de Banach, Ann. Inst. H. Poincaré 13, 161 (1953).

M 16, 268

Moy, S. T. C., Measure extensions and the martingale convergence theorem, Proc. Amer. Math. Soc. 4, 902 (1953).

M 15, 449

- Moyal, J. E., (See G. Dedebant) C. R. Acad. Sci. Paris 210, 243 (1940).

- Moyal, J. E., (See D. A. Edwards) Proc. Cambridge Philos. Soc. 51, 663 (1955). 
Nakagami, M., A method of calculating probability by means of differential operator, Nippon Elec. Commun. Engrg, No. 21, 28 (1940). M 2,229

Nakano, H., Über stochastischen Prozess. I, Proc. Imp. Acad. Tokyo 20, 513 (1944). M $\%, 313$

Nolfi, P., Wahrscheinlichkeit unstetiger Vorgänge bei kontinuierlich wirkenden Ursachen, Comment. Math. Helv. 15, 36 (1943).

M 6, 5

Onicescu, O., Les séries de structures, Acad. Roum. Bull. Sect. Sci. 26, 503 (1946).

M 9, 520

Onovama, T., On the linear translatable stochastic functional equation, Kōdai Math. Sem. Rep. No. 3. $33(1949)$.

M 11, 190

Onoyama, T., Regular random functions and linear translatable stochastic functional equations, $\mathrm{Mem}$. Fac. Sci., Kyūsyū Univ., Ser. A, 9, 135 (1956).

M 18, 156

Onoyama, T., Linear translatable stochastic functional equations and random distributions, $\mathrm{Mem}$. Fac. Sci., Kyūsyū Univ., Ser. A. 10, 29 (1956).

M 17, 1218

Ostene, E., Sur les zéros des matrices stochastiques, C. R. Acad. Sci. Paris 196, 150 (1933). Z 6, 68

Ottaviani, G., Sull'integrale stocastico, Cong. Mate. 610, Casa Editrice Perrella, Roma, 1953).

M 15, 19

Pal, L., On the theory of stochastic processes in cosmic radiation, Z. Eksper. Teoret. Fiz. 30, 362 (1956).

M 18, 76

Parzen, E., A central limit theorem for multilinear stochastic processes, Ann. Math. Statist. 28, 252 (1957).

M 18, 944

Patankar, V. N., The goodness of fit of frequency distributions obtained from stochastic processes, Biometrika 41, 450 (1954).

M 16, 731

Pennanéac'h, F., Fonctions aléatoires, Cahiers Rhodaniens 2, 20 (1950).

M 12, 840

Perfect, H., Methods of constructing certain stochastic matrices, Duke Math. J. 20, 395 (1953)

M 15, 3

-Pettis, B. J., (See N. Dunford) Proc. Nat. Acad. Sci. U.S.A. 52, 544 (1939).

-Pettis, B. J., (See N. Dunford) Trans. Amer. Math. Soc. 4\%, 323 (1940).

Philipson, C., A tentative application of the collective risk theory to crop insurance, Skand. Aktuarietidskr. 38, 201 (1955). M 18, 681

Philipson, C., A note on different models of stochastic processes dealt with in the collective theory of risk, Skand. Aktuarietidskr. 39, 26 (1956).

M 18, 681

-Pinsker, M. S., (See A. M. Yaglom) Doklady Akad. Nauk SSSR 90, 731 (1953).

Pitt, H. R., A special case of homogeneous random processes, J. London Math. Soc. 15, 247 (1940).

M 2,231

Pitt, H. R., Random processes in a group, J. London Math. Soc. 1\%, 88 (1942).

M 4, 219

Poliscuk, E. M., The mean value and integral of a functional, Ukrain. Mat. Z. 8, 59 (1956).

M 1\%, 1218
Pollaczek, F., Über zwei Formeln aus der Theorie des Wartens vor Schaltergruppen, Elektr. Nachr. Techn. 8, 256 (1931).

Z 2, 202

Pòlya, G., Theory of probability (Geneva Colloquium, 1938), Conf. Actual. Sci. Indus. Nr. 734 (Paris: Hermann \& Cie., 1938).

Z 22, 241

Pugačev, V. S., The general theory of correlation of random functions, Izvestiya Akad. Nauk SSSR. Ser. Mat. 1\%, 401 (1953).

M 15, 238

- Ramakrishnan, A., Sur les intégrales stochastiques associées aux processus ponctuels, Publ. Inst. Statist. Univ. Paris 5, 95 (1956). M 18, 680 -Rényi, A., (See L. Jánossy) Acta Math. Acad. Sci. Hungar. 1, 209 (1950).

- Rényi, A., Two proofs of a theorem of L. Jánossy, Magyar Tud. Akad. Mat. Fiz. Oszi. Közleményei 1, 369 (1951).

M 13, 957

Rényi, A., On composed Poisson distributions. II, Acta Math. Acad. Sci. Hungar. 2, 83 (1951).

M 13, 663

Ríos, S., Some probability laws and stochastic processes which reduce to a general type of Laplace-Stieltjes, Revista Mat. Hisp.-Amer. 13, 112 (1953).

M 14, 1101

Romanovskiǔ, V. I., On limiting distributions for stochastic processes with discrete time parameter, Acta Univ. Asiae Mediae, Fasc. 4, 25 (1945).

M 11, 189

Roseau, M., Sur une classe de fonctions aléatoires, C. R. Acad. Sci. Paris 230, 1497 (1950).

M 11, 732

Rosenblatt, A., Integration, for the case of a spherical cell, of the partial differential equation of diffusion encountered by the regular member of the Academy, Mr. Godofredo García, Revista Ci., Lima 46, 401 (1944).

M 6, 240

Rozenknop, I. Z., On some properties of collections of closed paths in a system with $n$ states and given connections among them, Izvestiya Akad. Nauk SSSR. Ser. Mat. 14, 95 (1950).

M 11, 445

Ruark, A., The time distribution of so-called random events, Phys. Rev. 56, 1165 (1939). M 1, 150

Ruark, A., Differential equations for the probability distribution of events, Phys. Rev. 65, 88 (1944).

M 6, 5

$\checkmark$ Rubin, H., (See T. W. Anderson) Ann. Math. Statist. 20, 46 (1949).

Saragina, Z. I., Local limit theorems for certain schemes of cyclic processes, Dokl. Akad. Nauk. SSSR. 110, 521 (1956).

M 18, 944

Sard, A., Smoothest approximation formulas, Ann. Math. Statist. 20, 612 (1949). M 12, 84

Sard, A., Approximation and projection, J. Math. Phys. 35, 127 (1956).

M 18, 322

Sarymsakov, T. A., Sur les suites des matrices stokhastiques, C.R. (Doklady) Acad. Sci. URSS 4\%, 326 (1945).

M $\boldsymbol{7}, 233$

-Savelli, M., (See A. Blanc-Lapierre) C. R. Acad. Sci. Paris 24\%, 2799 (1956). 
Savkevitch, V., Sur le schéma des urnes à composition variable, C. R. (Doklady) Acad. Sci. URSS. 28, 8 (1940).

M $\boldsymbol{2}, 229$

Savkevitch, V., Schèma de l'urne aux boules surajoutées, Leningrad State Univ. Annals 83, 129 (1941).

M $\boldsymbol{\gamma}, 459$

Schützenberger, M. P., Remarques sur des relations d'ordre entre variables aléatoires indépendants, C. R. Acad. Sci. Paris 224, 878 (1947). M 8, 472

Ščigolev, B., On differential equations of the 1st order with a random term, Moskov. Gos. Univ. Trudy Gos. Astr. Inst. 24, 93 (1954). $\quad$ M 16, 938

Seal, H. L., Discrete random processes, J. Inst. Actuaries Students' Soc. 8, 204 (1949).

M 11, 120

Siegert, A. J. F., On the first passage time probability problem, Physical Rev. 81, 617 (1951).

M 14, 887

Silberstein, L., On a hystero-differential equation arising in a probability problem, Philos. Mag. 29, $75(1940)$.

M 1, 150

Slutsky, E., Quelques propositions sur la théorie des fonctions aléatoires, Acta Univ. Asiae Mediae. Ser. V. 31, 15 pp. (1939).

M 9, 46

Smirnov, N. V., On the distribution of the number of cycles in cyclic systems, Uspehi Matem. Nauk 4, 192 (1949).

M 11, 189

Smith, W. L., Regenerative stochastic processes, Proc. Roy. Soc. London Ser. A. 232, 6 (1955).

M 1\%, 502

४ Snell, J. L., (See J. G. Kemeny) (Prentice Hall, Englewood Cliffs, N.J., 1957).

Špaček, A., Zufällige Gleichungen, Crechoslovak Math. J. 5, 80, 462 (1955).

M 18, 157

Špaček, A., Die Regularitätseigenschaften zufälliger Transformationen, Ber U Wahrsch Stat (Verlag Wissenschaften Berlin 1956).

M 18, 605

- Srinivasan, S. K. (See A. Ramakrishnan), Publ. Inst. Statist. Univ. Paris 5, 95 (1956).

- Srinivasan, S. K. (See P. M. Mathews), Proc. Indian Acad. Sci. Sect. A. 43, 4 (1956).

- Stratonovic, R. L. (See P. I. Kuznecov), Akad. Nauk SSSR. Žurnal Tehn. Fiz. 24, 103 (1954).

- Stratonovic, R. L. (See P. I. Kuznecov), Izv. Akad. Nauk SSSR. Ser. Mat. 20, 167 (1956).

Takacs, L., A new method for discussing recurrent stochastic processes, Magyar Tud. Akad. Alkalm. Mat. Int. Közl. 2, 135 (1954).

M 16, 379

Takacs, L., Some investigations concerning recurrent stochastic processes of a certain type, Magyar Tud. Akad. Alkalm Mat. Int. Közl. 3, 115 (1955).

M 1\%, 866

Takacs, L., On secondary stochastic processes derived by means of recurrence processes, Magyar Tud. Akad. Mat. Fiz. Oszt. Közl. 5, 187 (1955).

M 1\%, 276

Takacs, L., On the sequence of events, selected by a counter from a recurrent process of events, Teor. Veroyatnost. I Primenen. 1, 90 (1956). M 18, 831

Tchen, C. M., Stochastic processes and dispersion of configurations of linked events, J. Research Nat. Bur. Standards 46, 480 (1951).

M 13, 141
-Thompson, G. L. (See J. G. Kemeny) (Prentice Hall, Englewood Cliffs, N.J., 1957).

-Tihonov, V. I. (See P. I. Kuznecov), Akad. Nauk SSSR. Žurnal Tehn. Fiz. 24, 103 (1954).

- Turán, P. (See A. Rényi), Magyar Tud. Akad. Mat. Fiz. Oszt. Közleményei 1, 369 (1951).

Udeschini, P., Meccanica aleatoria, Rend. Sem. Mat. Fis. Milano 20, 54 (1950).

M 12, 620

Vogel, W., Asymptotische Eigenschaften von Maximum-Likelihood Schätzwerten bei einem stochastischen Prozess, Monatsh. Math. 60, 313 (1956).

M 18, 682

Wald, A., Asymptotic properties of the maximum likelihood estimate of an unknown parameter of a discrete stochastic process, Ann. Math. Statist. 19, 40 (1948). M 9, 454

-Wehrlé, P. (See G. Dedebant), C. R. Acad. Sci. Paris 210, 243 (1940).

-Wehrlé, P. (See G. Dedebant), Portugaliae Phys. 1, 95 (1944).

-Wehrlé, P. (See J. Bass), C. R. Acad. Sci. Paris 221, 168 (1945).

Wiener, N., The homogeneous chaos, Amer. J. Math. 60, 897 (1938).

Z 19, 354

Wiener, N., Sur la théorie de la prévision statistique et du filtrage des ondes, Colloq. Int. Centre Nat. Rech., 15, 67, Paris (1949).

M 11, 376

Wiener, N., Optics and the theory of stochastic processes, J. Opt. Soc. Amer. 43, 225 (1953).

M 17, 33

Wintner, A. (See P. Hartman), Amer. J. Math. 61, 977 (1939).

-Wintner, A. (See P. Hartman), Amer. J. Math. 64, 273 (1942).

Wold, H., UUber stochastische Prozesse, insbesondere solche stationärer Natur, Congr. des. Math. Scand. 207-218 (1939).

Z 21, 41

-Wolfowitz, J. (See Kiefer, J.), Ann. Math. Statist. 2\%, 147 (1956).

- Yaglom, A. M., Random processes with stationary increments of the $n$th order, Doklady Akad. Nauk. SSSR 90, 731 (1953).

M 15, 238

Yosida, K., On the Fokker-Planck equation and its integral, Sügaku 3, 129 (1951). $\quad$ M 14, 1089

Yosida, K., On the Fokker-Planck equation and its integral, II, Sūgaku 4, 145 (1952). M 14, 1089

Zadeh, L. A., On a class of stochastic operators, J. Math. Physics 32, 48 (1953). M 14, 771

\section{B. Markov Processes}

Anderson, T. W., Statistical inference about Markov chains, Ann. Math. Statist. 28, 89 (1957).

M 18, 944

Arbey, L., Les erreurs d'observations considérées comme liées, Bull. Astr. 14, 75 (1949).

M 12, 347 
Austin, D. G., Some differentiation properties of Markoff transition probability functions, Prce. Amer. Math. Soc. 7, 751 (1956).

M 18, 424

Barkalaia, A., Sur des chaînes de Markoff, Uchenye Zapiski Moskov. Gos. u. Matematika 73, 33 (1944).

M $\boldsymbol{7}, 210$

Bartlett, M. S., Recurrence times, Nature 165, 727 (1950).

M 11, 671

Bartlett, M. S., The frequency goodness of fit test for probability chains, Proc. Cambridge Philos. Soc. 47, 86 (1951).

M 12, 512

Bartlett, M. S., A sampling test of the $\chi^{2}$ theory for probability chains, Biometrika 39, 118 (1952).

M 13, 962

- Bass, J., Sur certains classes de fonctions aléatoires, C. R. Acad. Sci. Paris 22\%, 1206 (1948).

M 10, 385

Batchelor, G. K., Diffusion in a field of homogeneous turbulence. I. Eulerian analysis, Australian J. Sci. Research. Ser. A. 2, 437 (1949). M 12, 451

Bateman, G., On the power function of the longest run as a test for randomness in a sequence of alternatives, Biometrika 35, 97 (1948). M 9, 603

Beboutoff, M., Markoff chains with a compact state space, C.R. (Doklady) Acad. Sci URSS 30, 482 (1941).

M 3, 3

Beboutoff, M., Markoff chains with a compact state space, Rec. Math. [Mat. Sbornik] 10, 213 (1942).

M $\boldsymbol{\%}, 19$

Bellman, R., On a generalization of the fundamental identity of Wald, Proc. Cambridge Philos. Soc. 53, 258 (1957).

M 18, 681

Bernardelli, H., The stability of the income distribution, Sankhyā 6, 351 (1944).

M 6, 94

Bernstein, S., Sur les chaînes linéaires de Markov quasi-continues, C. R. Acad. Sci. URSS, 1, 1 (1934).

Z 8, 265

Bernstein, S., Sur les chaînes linéaires de Markov quasi-continues, C.R. Acad. Sci. URSS 1, 361 (1934).

Z 8, 399

Bernstein, S., Détermination d'une limite inférieure de la dispersion des sommes de grandeurs liées en chaîne singulière, Rec. Math. Moscou, 1, 29 (1936).

Z 13, 408

Bityuckov, V. I., A local limit theorem for sequences of events forming a compound chain of the second order, Izvestiya Akad. Nauk SSSR. Ser. Mat. 12, 101 (1948).

M 9, 451

Blackwell, D., Idempotent Markoff chains, Ann. of Math. 43, 560 (1942).

M 4, 17

Blackwell, D., Finite non-homogeneous chains, Ann. of Math. 46, 594 (1945).

M $\boldsymbol{\gamma}, 210$

Blackwell, D., The existence of anormal chains, Bull. Am. Math. Soc. 51, 465 (1945). M 6, 233

Blackwell, D., Extension of a renewal theorem, Pacific J. Math. 3, 315 (1953).

M 14, 944

Blackwell, D., On transient Markov processes with a countable number of states and stationary transition probabilities, Ann. Math. Statist. 26, 654 (1955).

M 18, 754
Bochner, S., Diffusion equation and stochastic processes, Proc. Nat. Acad. Sci. U.S.A., 35, 368 (1949).

M 10, 720

$\checkmark$ Bogoliouboff, N., (See N. Kryloff) C. R. Acad. Sci., Paris, 204, 1386 (1937).

Borel, E., Sur les séquences en météorologie, Ann. du Bureau des Longitudes (1948).

M 10, 200

Brier, G. W., The statistical theory of turbulence and the problem of diffusion in the atmosphere, $J$. Meteorol., $\%, 283$ (1950).

M 12, 299

Castoldi, L., Irreversibilità delle catene ordinarie di Markov, Boll. Un. Mat. Ital. 11, 16 (1956).

M 1\%, 1218

$\checkmark$ Cernuschi, F., A new scheme of contagion in probability, An. Soc. Ci, Argentina 138, 201 (1944).

M 6, 233

Chung, K. L., Contributions to the theory of Markov chains, J. Research Nat. Bur. Standards 50, 203 (1953).

M 14, 1099

Chung, K. L., Contributions to the theory of Markov chains. II, Trans. Amer. Math. Soc. $\mathbf{g 6 ,} 397$ (1954).

M 16, 149

Chung, K. I., Foundations of the theory of continuous parameter Markov chains, Proc. Symp. Stat. Prob., 11, 29 (Univ. Calif. Press, Berkeley, 1956).

M 18, 940

Chung, K. L., Some new developments in Markov chains, Trans. Amer. Math. Soc. 81, 195 (1956)

M 17, 755

Ciucu, G., Chaînes à liaisons complètes du type, Com. Acad. R. P. Romîne 1, 455 (1951). M 18,50

Ciucu, G., Chaînes à liaisons complètes à densité, Com. Acad. R. P. Romîne 4, 345 (1954). M 1\%, 50

Clarke, A. B., A waiting line process of Markov type, Ann. Math. Statist. 2\%, 452 (1956). M 18, 157

Consael, R., Sur certaines équations fonctionnelles de la théorie des processus markoviens, Assoc. Actuair. Beiges. Bull No 5\%, 63 (1954) M 16, 1036

Culanovskiľ, I. V., On cycles in Markov chains, Doklady Akad. Navk SSSR, 69, 301 (1949)

M 11, 256

Daniels, H. E., The approximate distribution of serial correlation coefficients, Biometrika 43, 169 (1956).

M 18, 79

Daniels, H. E., The statistical theory of stiff chains, Proc. Roy. Soc. Edinburgh. Sect. A., 63, 290 (1952).

M 14, 295

van Dantzig, D., Chaînes de Markof dans les ensembles abstraits et applications aux processus avec régions absorbantes et au problème des boucles, Ann. Inst. H. Poincaré 14, 145 (1955).

M 1\%, 867

- van Dantzig, D., On arbitrary hereditary timediscrete stochastic processes, considered as stationary Markov chains, and the corresponding general form of Wald's fundamental identity, Nederl. Akad. Wetensch. Proc. Ser. A., 5\%, 377 (1954).

M 16, 379

Darling, D. A., The first passage problem for a continuous Markov process, Ann. Math. Statist., 24, 624 (1953).
M 15, 449 
-Darling, D. A., On the distribution of certain functionals of Markoff chains and processes, Proc. Nat. Acad. Sci. U.S.A., 42, 525 (1956). M 18, 155 -Darling, D. A., On occupation times for Markoff processes, Trans. Amer. Math. Soc. 84, 444 (1957).

M 18, 832

Davidson, A., On the problem of ruin in the collective risk theory under the assumption of variable safety loading, Försäkringsmatematiska Studier, pp 32-47 (Stockholm 1946).

M 8, 390

Derman, C., A solution to a set of fundamental equations in Markov chains, Proc. Amer. Math. Soc. 5, 332 (1954).

M 15, 722

Derman, C., Some contributions to the theory of denumerable Markov chains, Trans. Amer. Math. Soc. 79, 541 (1955).

$\mathrm{M} \mathbf{1 \%}, 50$

Doblin, V., Sur des mouvements mixtes, C. R. Acad. Sci. Paris 210, 690 (1940)

M $\boldsymbol{2}, 230$

Doblin, W., Eléments d'une théorie générale des chaînes simples constantes de Markoff, Ann. École Norm. 5\%, 61 (1940).

M 3, 3

Dobrušin, R. L., Limit theorems for a Markov chain of two states, Izvestiya Akad. Nauk SSSR. Ser. Mat. 17, 291 (1953).

M 15, 329

Dobrušin, R. L., Generalization of Kolmogorov's equations for Markov processes with a finite number of possible states, Mat. Sbornik 33, 567 (1953).

M 15, 542

Dobrušin, R. L., Conditions of regularity of Markov processes with a finite number of possible states, Mat. Sbornik 34, 541 (1954)

M 16, 150

Dobrušin, R. L., On the condition of the central limit theorem for inhomogeneous Markov chains, Dokl. Akad. Nauk SSSR. 108, 1004 (1956). M 18, 424

Doeblin, W., Sur les chaînes discrètes de Markoff, C. R. Acad. Sci., Paris 203, 24 (1936). Z 14, 222

Doeblin, W., Sur les chaînes de Markoff, C. R. Acad. Sci., Paris 203, 1210 (1936).

Z 15, 262

Doeblin, W. Sur le cas continu des probabilités en chaîne, Atti Accad. naz. Lincei, Rend., 25, 170 (1937).

Z 16, 311

Doeblin, W., Sur les propriétés asymptotiques de mouvement régis par certains types de chaînes simples, Bull. Math. Soc. Roum. Sci. 39, 57 (1937).

Z 19, 175

-Doeblin, W., Sur des chaînes à liaisons complètes, Bull. Soc. Math. France 65, 132 (1937). Z 18, 33

Doeblin, W., Eléments d'une théorie générale des chaînes constantes simples de Markoff, C.R. Acad. Sci., Paris 205, 7 (1937).

$\mathrm{Z} \mathbf{1 \%}, 77$

Doeblin. W., Le cas discontinu des probabilités en chaîne, Publ. Fac. Sci. Univ. Masaryk 236, 3 (1937).

Z 16, 411

Doeblin, W., Exposé de la théorie des chaînes simples constantes de Markoff à un nombre fini d'états, Rev. Math. Union Interbalkan, 2, 77 (1938).

Z 21, 422

Doeblin, W., Sur certains mouvements aléatoires discontinus, Skand. Aktuarietidskr. 1939, 211 (1939).

M 1, 247

Doeblin, W., Eléments d'une théorie générale des chaînes simples constantes de Markoff, Ann. École Norm., 5\%, 61 (1940).

$\mathrm{Z} \mathbf{2 4}, 265$
Doeblin, W., Sur l'équation de Kolmogoroff, $C . R$. Acad. Sci. Paris 210, 365 (1940). M 1, 343

Doob, J. L., Topics in the theory of Markoff chains, Trans. Amer. Math. Soc. 52, 37 (1942). M 4, 17

Doob, J. L., Markoff chains - denumerable case, Trans. Amer. Math. Soc. 58, 455 (1945). M g, 210

Doob, J. L., Asymptotic properties of Markoff transition probabilities, Trans. Amer. Math. Soc. 63, 393 (1948).

M 9, 598

Doob, J. L., Martingales and one-dimensional diffusion, Trans. Amer. Math. Soc. 88, 168 (1955).

M 1\%, 50

Doob, J. L., A probability approach to the heat equation, Trans. Amer. Math. Soc. 80, 216 (1955).

M 18, 76

Doob, J. L., Probability methods applied to the first boundary value problem, Proc. Symp. Stat. Prob., II, 49 (Univ. Calif. Press, Berkeley 1956)

M 18, 941

Dubrovsky, V. M., On a problem connected with purely discontinuous random processes, $C . R$. Acad. Sci. URSS. 4\%, 459 (1945).

M $\boldsymbol{7}, 210$

Dynkin, E. B., Criteria of continuity and of absence of discontinuities of the second kind of trajectories of a Markov random process, Izvestiya Akad. Nauk SSSR. Ser. Mat. 16, 563 (1952). M 14, 567

Dynkin, E. B., On some limit theorems for Markov chains, Ukrain. Mat. Z. 6, 21 (1954). M 17, 865

Dynkin, E. B., On new analytic methods in the theory of Markov random processes, Vestnik Leningrad. Univ. 10, 69 (1955). $\quad$ M 17,754

Dynkin, E. B., Functionals of trajectories of Markov random processes, Dokl. Akad. Nauk SSSR. 104, 691 (1955).

M 17, 501

Dynkin, E. B., Infinitesimal operators of Markov random processes, Dokl. Akad. Nauk SSSR. 105, 206 (1955).

M 1\%, 866

Dynkin, E. B., Continuous one-dimensional Markov processes, Dokl. Akad. Nauk SSSR. 105, 405 (1955).

M 1\%, 865

Eberl, W., Ein Zufallsweg in einer Markoffschen Kette von Alternativen, Monatsh. Math. 58, 137 (1954)

M 16, 379

Eberl, W., Die Summenverteilung verketteter Alternativen, Österreich. Ing.-Arch. 9, 280 (1955).

M 1\%, 501

Elfving, G., Zur Theorie der Markoffschen Ketten, Acta Soc. Sci. Fennicae, 2, 1 (1937) Z 1\%, 316

Elfving, G., Über die Interpolation von Markoffschen Ketten, Soc. Sci. Fennica. Comment. Phys.-Math. 10, 1 (1938).

Z 18, 264

Elfving, G., Contributions to the theory of integervalued Markoff processes, Skand. Aktuarietidskr. 29, 175 (1946).

M 8, 391

Elfving, G., On a class of elementary Markoff processes, C. R. Cong. Math. Scandinaves (Jul. G. Forlag, Copenhagen 1947). M 8, 472

Epstein, B., The distribution of extreme values in samples whose members are subject to a Markoff chain condition, Ann. Math. Statist. 20, 590 (1949).

M 11, 375 
Feldheim, E., Sur les probabilités en chaîne, Math. Ann. 112, 775 (1936).

$\mathrm{Z}$ 14, 28

Feller, W., On the integro-differential equations of purely discontinuous Markoff processes, Trans. Amer. Math. Soc. 48, 488 (1940).

M 2, 101

Feller, W., The problem of $\mathrm{n}$ liars and Markov chains, Amer. Math. Monthly 58, 606 (1951).

M 13, 363

Feller, W., Diffusion processes in one dimension, Trans. Amer. Math. Soc. 78, 1 (1954). M 16, 150

- Fennell, J., The dynamics of overhaul and replenishment systems for large equipments, Naval Res. Logist. Quart. 3, 19 (1956).

M 18, 451

Fortet, R., Sur des probabilités en chaîne, $C$. $R$. Acad. Sci., Paris 201, 184 (1935). Z 12, 28

Fortet, R., Sur des probabilités en chaîne, $C$. $R$. Acad. Sci., Paris 202, 1362 (1936). Z 14, 28

Fortet, R. (See W. Doeblin), Bull. Soc. Math. France 65, 132 (1937).

Z 18, 33

Fortet, R., Sur l'itération des substitutions algébriques linéaires à une infinité de variables et ses applications à la théorie des probabilités en chaîne, Rev. Ci, Lima 40, 185 (1938).

Z 21, 388

Fortet, R., Sur des fonctions aléatoires définies par leurs équations aux dérivées partielles, $C$. $R$. Acad. Sci. Paris 212, 325 (1941).

M 3, 4

Fortet, R., Sur le calcul de certaines probabilités d'absorption, C. R. Acad. Sci. Paris 212, 1118 (1941).

M 5, 125

Fortet, R., Les fonctions aléatoires du type de Markoff associées à certaines équations linéaires aux dérivées partielles du type parabolique, $J$. Math. Pures Appl. 22, 177 (1943).

M $\boldsymbol{\gamma}, 19$

Foster, F. G., Markoff chains with an enumerable number of states and a class of cascade processes, Proc. Cambridge Philos. Soc. 4\%, 77 (1951).

M 12, 620

Foster, F. G., A Markov chain derivation of discrete distributions, Ann. Math. Statist. 23, 624 (1952).

M 14, 663

Foster, F. G., On Markov chains with an enumerable infinity of states, Proc. Cambridge Philos. Soc. 48, 587 (1952).

M 14, 295

Fouillade, A., Sur une conception de la théorie des probabilités en chaîne, Bull. Sci. Math., II. 61, 269 (1937).

$\mathrm{Z}$ 18, 33

Franckx, E., La théorie des chaînes de Markoff. Étude du cas régulier, Skand. Aktuarietidskr. 1939, $200(1939)$.

M 1, 342

Franckx, E., Relation entre les ensembles renouvelés et les probabilités en chaîne, $C$. R. Acad. Sci. Paris \$30, 359 (1950).

M 11, 376

Franckx, E., La génération d'une chaîne de Markoff, Mitt. Verein. Schweiz. Versich.-Math. 53, 145 (1953).

M 15, 238

Fréchet, M., Solution continue la plus générale d'une équation fonctionnelle de la théorie des probabilités "en chaîne," Bull. Soc. Math. France 60, 242 (1932).

$\mathrm{Z} \mathbf{6}, 173$

Fréchet, M., Sur le comportement de certains noyaux de Fredholm itérés indéfiniment et sur les probabilités en chaîne, C. R. Acad. Sci., Paris 195, 590 (1932).

$\mathrm{Z} \mathbf{5}, 254$
Fréchet, M., Compléments à la théorie des probabilités discontinues "en chaînes," Ann. Scuola Norm. Super. Pisa, II. 2, 131 (1933). Z 6, 67 Fréchet, M., Les probabilités continues "en chaîne," Comment. Math. Helv. 5, 175 (1933). Z Z 6, 357

$\checkmark$ Fréchet, M., (See J. Hadamard), Z. Agnew. Math. Mech. 13, 92 (1933).

Fréchet, M., Solution continue la plus générale d'une équation fonctionnelle de la théorie des probabilités en chaîne (Note complémentaire.), Bull. Soc. Math. France 61, 182 (1933) Z 8, 120

Fréchet, M., Sur l'équation fonctionnelle de S. Chapman et sur les problémes des probabilités en chaîne, Atti Accad. naz Lincei. Rend. VI 20, 95 (1934)

$\mathrm{Z}$ 10, 70

Fréchet, M., Sur l'allure asymptotique des densités itérées dans le probléme des probabilités "en chaîne," Bull. Soc. Math. France 62, 68 (1934)

$\mathrm{Z} \mathbf{9}, 264$

Fréchet, M., Sur l'équation fonctionnelle de Chapman et sur le probléme des probabilités en chaîne, Proc. London Math. Soc. II 39, 515 (1935)

Z 12, 111

Fréchet, M., Sul caso positivamente regolare nel problema delle probabilità concatenate, Giorn. Ist. Ital. Attuari \%, 28 (1936)

Z 13, 313

Fuchs, A., Sur quelques points de la théorie des processus de Markoff presque sûrement continus dans un intervalle, C. R. Acad. Sci. Paris 23\%, 1137 (1953)

M 15, 330

Fuchs, A., Sur la continuité stochastique des processus stochastiques réels de Markoff, $C$. R. Acad. Sci. Paris 23\%, 1388 (1953)

M 15, 330

Fuchs, A., Sur un théorème de N. Wiener, $C$. $R$. Acad. Sci. Paris 240, 1396 (1955) M 16, 839

Fuchs, A., Sur certains opérateurs linéaires associés aux processus réels de Markoff, C. R. Acad. Sci. Paris 240, 1506 (1955)

M 16, 839

- Fuchs, A., Tendance vers un état d'équilibre stable de phénomènes soumis à une évolution markovienne, C. R. Acad. Sci. Paris 242, 1120 (1956)

M 18, 76

Gani, J., The condition of regularity in simple Markov chains, Austral. J. Phys. 9, 387 (1956).

M 18, 520

Gani, J., Sufficiency conditions in regular Markov chains and certain random walks, Biometrika $\mathbf{4 3}$, 276 (1956).

M 18, 342

Ghermănescu, M., Sur les chaînes de Markov, Acad. R. P. Romîne, Bul. Sect. Mat. Fiz. 8, 101 (1956).

M 18, 944

Gihman, I. I., Markov processes in problems of mathematical statisties, Ukrain. Mat. Ž. 6, 28 (1954)

M 1\%,980

Gihman, I. I., On asymptotic properties of certain statistics similar to $\chi^{2}$, Teor. Veroyatnost. $i$ Primenen, 1, 344 (1956).

M 18, 945

Gnedenko, B., Investigation of the growth of homogeneous random processes with independent increments, C. R. (Doklady) Acad. Sci. URSS 36, 3 (1942)
M 4, 103 
Gnedenko, B. V., Limit theorems for sums of independent summands and for Markov chains, Ukrain. Mat. Ž. 6, 5 (1954)

M 1\%, 978

Gnedenko, B. V., On the iterated logarithm law for homogeneous random processes with independent increments, C. R. (Doklady) Acad. Sci. URSS 40, 255 (1943)

M 6, 88

$\checkmark$ Goodman, L. A., (See T. W. Anderson) Ann. Math. Statist. 28, 89 (1957).

- Hadamard, J., Sur les probabilités discontinues des évènements "en chaîne," $Z$. angew. Math. Mech. 13, 92 (1933). Z 6, 357

Hajnal, J., The ergodic properties of non-homogeneous finite Markov chains, Proc. Cambridge Philos. Soc. 52, 67 (1956).

M 17, 501

Hannan, E. J., Exact tests for serial correlation in vector processes, Proc. Cambridge Philos. Soc. 52, $482(1956)$.

M 18, 520

Harris, T. E., First passage and recurrence distributions, Trans. Amer. Math. Soc. 73, 471 (1952).

M 14, 567

-Harris, T. E., Ergodic theory of Markov chains admitting an infinite invariant measure, Proc. Nat. Acad. Sci. U.S.A. 39, 860 (1953) M 15, 140

Harris, T. E., On chains of infinite order, Pacific $J$. Math. 5, 707 (1955).

M 1\%, 755

Harris, T. E., The existence of stationary measures for certain Markov processes, Proc. Symp. Stat. Prob., II, 113 (Univ. Calif. Press, Berkeley 1956).

M 18, 941

Hetz, W., Untersuchungen zum Erneuerungsproblem, insbesondere seine Darstellung als Markoffsche Kette, Bl. Deutsch. Ges. Versicherungsmath. 3, 23 $(1956)$.

M 18, 548

Hida, T., On the transition probability of a renewal process, Nagoya Math. J. 11, 41 (1957).

M 18, 945

Hille, E., Les probabilités continues en chaîne, $C . R$. Acad. Sci., Paris \$30, 34 (1950). $\quad$ M 11, 256

Hittmair, O., Valeur extrémale des distributions de probabilités conditionnelles dans une chaîne de Markoff, C. R. Acad. Sci. Paris 238, 1469 (1954).

M 15, 723

Homma, T., On the theory of queues with some types of queue-discipline, Yokohama Math. J. 4, 55 (1956).

M 18, 770

Hopf, E., The general temporally discrete Markoff process, J. Rational Mech. Anal. 3, 13 (1954).

M 15, 636

-Horton, H. B., A direct method for producing random digits in any number system, Ann. Math. Statist. 20, 82 (1949).

M 10, 550

Hostinsky, B., Sur la théorie des chaînes de Markoff et sur l'intégration des transformations linéaires, Cas. Mat. Fys. 63, 167 (1934).

Z 9, 27

Hostinsky, B., Chaînes de Markoff inverses, Acad. Tchèque. Sci., Bull. Int. 36, 60 (1935). Z 16, 312

Hostinsky, B., Chaînes de Markoff inverses, Acad. Tchèque. Sci., Bull. Int. 36, 64 (1935). Z 16, 312

Hostinsky, B., Sur les probabilités en chaîne, C. R. Acad. Sci., Paris 202, 1000 (1936). Z 13, 313
Hostinsky, B., Sur les mouvements qui dépendent du hasard, C. R. Acad. Sci., Paris 202, 2029 (1936).

$\mathrm{Z}$ 14, 168

Hostinsky, B., Sur les probabilités relatives aux variables aléatoires liées entre elles, Applications diverses, Ann. Inst. H. Poincaré \%, 69 (1937).

$\mathrm{Z} \mathbf{1 8}, 77$

Hostinsky, B., Sulle successioni di variabili casuali, Giorn.1 st Ital. Attuari. 8, 8-13 (1937). Z 16, 171

Hostinsky, B., Probabilités relatives aux tirages de deux urnes avec l'échange des boules extraites, Acta Univ. Asiae Mediae. 21, (1939). M 8, 522

Hostinsky, B., Equations fonctionnelles relatives aux probabilités continues en chaîne, (Actualitès Sci. Ind. 782; Paris, Hermann \& Cie. 1939). Z Z 21, 422

Hostinsky, B., Le principe ergodique et les probabilités en châ̂ne, (Actualitès Sci. \& Ind. $73 \%$; Paris, Hermann \& Cie 1938). Z Z 22, 242

Hostinsky, B., Sur le coefficient de corrélation, Memorial Volume Dedicated to D. A. Grave, $p p$. 48-51 (Moscow 1940).

M 2,229

Hostinsky, B., Revue des travaux publiés en 19351948 sur les chaînes de Markoff et problèmes voisins, Casopis Pěst. Mat. Fys. \%4, 48 (1949).

M 12, 425

Hunt, G. A., Some theorems concerning Brownian motion, Trans. Amer. Math. Soc. 81, 294 (1956).

M 18, 77

Ionescu, H., Two applications of probability calculus, Gaz. Mat. Fiz. Ser. A. 8, 225 (1956).

M 18, 343

Itô, K., On stochastic differential equations, Mem. Amer. Math. Soc., No. 4, (1951). M 12, 724

Itô, S., Brownian motions in a topological group and in its covering group, Rend. Circ. Mat. Palermo 1, 40 (1952).

M 14, 187

Janossy, L., Generalized form of the diffusion equation for a single particle, Ž. Eksper. Teoret. Fiz. 30, 351 (1956).

M 18, 76

Jensen, A., Distribution patterns, composed of a limited number of exponential distributions, Skandinaviske Matemat. Kong. p. $\mathbf{2 1 0}$ (Forlag, Oslo, 1952).

M 14, 664

Jensen, A., Application of stochastic processes to an investment plan, Metroecon. 5, 129 (1953).

M 18, 241

Jensen, A., Markoff chains as an aid in the study of Markoff processes, Skand. Aktuarietidskr. 36, 87 (1953).

M 15, 238

Kac, M., Random walk and the theory of Brownian motion, Amer. Math. Monthly 54, 369 (1947).

M 9, 46

$\checkmark$ Kac, M., (See D. A. Darling), Trans. Am. Math. Soc. 84, 444 (1957).

Kakutani, S., Some results in the operator-theoretical treatment of the Markoff process, Proc. Imp. Acad., Tokyo 15, 260 (1939). Z 22, $246 ;$ M 1, 150 
-Kakutani, S., (See K. Yosida), Jap. J. Math. 16, 47 (1939).

Kakutani, S., Ergodic theorems and the Markoff process with a stable distribution, Proc. Imp. Acad. Tokyo 16, 49 (1940).

M 1, 343

-Kakutani, S., (See K. Yosida), Ann. of Math. 4\%, 188 (1941).

Kakutani, S., Markoff process and the Dirichlet problem, Proc. Japan Acad. 21, 227 (1945).

M 11, 357

Kakutani, S., Random ergodic theorems and Markoff processes with a stable distribution, Proc. Symp. Math. Prob., p. 247 (Univ. Calif. Press, Berkeley, 1951).

M 13, 476

Kallianpur, G., On an ergodic property of a certain class of Markov processes, Proc. Amer. Math. Soc. 6, 159 (1955).

M 16, 1035

Karlin, S., Some random walks arising in learning models, I., Pacific J. Math. 3, 725 (1953).

M 15, 450

Kazami, A., Asymptotic properties of the estimates of an unknown parameter in stationary Markoff process, Ann. Inst. Statist. Math., Tokyo 4, 1 (1952).

M 14, 569

Keilson, J., A suggested modification of noise theory, Quart. Appl. Math. 12, 71 (1954).

M 18, 605

- Kemeny, J. G., Introduction to finite mathematics (Prentice Hall, Englewood Cliffs, N.J., 1957).

M 18, 860

Kendall, D. G., On non-dissipative Markoff chains with an enumerable infinity of states, Proc. Cambridge Philos. Soc. 4\%, 633 (1951). M 13, 51

Kinney, J. R., Continuity properties of sample functions of Markov processes, Trans. Amer. Math. Soc. \%4, 280 (1953).

M 14, 772

Kolmogoroff, A., Zufällige Bewegungen (Zur Theorie der Brownschen Bewegung), Ann. of Math., II. 35, 116 (1934).

Z 8, 399

Kolmogoroff, A., Zur Theorie der Markoffschen Ketten, Math. Ann. 112, 155 (1935). Z 12, 410

Kolmogoroff, A., Anfangsgründe der Theorie der Markoffschen Ketten mit unendlich vielen möglichen Zuständen, Rec. Math. Moscou, 1, 607 (1936).

Z 15, 219

Kolmogoroff, A., Chaînes de Markoff avec une infinite dénombrable des états possibles, Bull. Univ. État. Moscou, Sér. Int., Sect. A. 1, 1 (1937).

Z 18, 413

Kolmogorov, A. N., A local limit theorem for classical Markov chains, Izvestiya Akad. Nauk SSSR. Ser. Mat. 13, 281 (1949).

M 11, 119

Kolmogorov, A. N., On the differentiability of the transition probabilities in stationary Markov processes with a denumberable number of states, Moskov, Gos. Univ. Učenye Zapiski 148, Mat. 4, 53 (1951).

M 14, 295

Koopman, B. O., A generalization of Poisson's distribution for Markoff chains, Proc. Nat. Acad. Sci. U.S.A. 36, 202 (1950).

M 11, 445

Koopman, B. O., A law of small numbers in Markoff chains, Trans. Amer. Math. Soc. 90, 277 (1951).

M 14, 1100
Krishna, P. V., Factorial moments and cumulants of distributions arising in Markoff chains, $J$. Indian Soc. Agric. Statist. 4, 113 (1952). M 14, 771

-Kryloff, N., Sur les probabilités en chaîne, $C$. $R$. Acad. Sci., Paris 204, 1386 (1937). $\quad$ Z 16, 312

Kunisawa, K., On the mixed Markoff process, Kōdai Math. Sem. Rep., No. 3, 28 (1949).

M 11, 189

Labutin, D. N., On a generating function, Leningrad State Univ. Annals Math. Ser. 10, 139 (1940).

M 2,229

$\checkmark$ Le Cam, L., (See J. Bass), C. R. Acad. Sci. Paris 22\%, 1206 (1948).

Ledermann, W., On the asymptotic probability distribution for certain Markoff processes, Proc. Cambridge Philos. Soc. 46, 581 (1950). M 12, 269

Ledermann, W., Corrigendum to the paper "On the asymptotic probability distribution for certain Markoff processes," Proc. Cambridge Philos. Soc. 4\%, 626 (1951).

M 12, 840

-Ledermann, W. (See G. E. H. Reuter), Proc. Cambridge Philos. Soc. 49, 247 (1953).

Lévy, P., Propriétés asymptotiques des sommes de variables aléatoires enchaînees, $C$. R. Acad. Sci., Paris 199, 627 (1934).

$\mathrm{Z}$ 10, 70

Lévy, P., La loi forte des grands nombres pour les variables aléatoires enchaînées, J. Math. Pures Appl., IX 15, 11 (1936).

$\mathrm{Z}$ 13, 273

Lévy, P., Exemples de processus doubles de Markoff, C. R. Acad. Sci. Paris 226, 307 (1948) M 9, 361

Lévy, P., Chaînes doubles de Markoff et fonctions aléatoires de deux variables, C. R. Acad. Sci. Paris 226, 53 (1948).

M 9, 361

Lévy, P., Processus doubles de Markoff. Le Calcul des Probabilités et ses Applications, Colloq. Inter. Centre Nat. Rech. Sci., No. 13, 53 Paris (1949).

M 12, 114

Lévy, P., Exemples de processus pseudo-markoviens, C. R. Acad. Sci. Paris 228, 2004 (1949). Lévy, P., Deux nouveaux exemples de processus stochastiques, C. R. Acad. Sci. Paris P31, 1208 (1950).

Lévy, P., Fonctions aléatoires $H(t)$ à valeurs entières, dépendant de processus à la fois markoviens et stationnaires, C. R. Acad. Sci. Paris 232, 1400 (1951).

M 12, 723

Lévy, P., Processus de Markoff. Cas dénombrable, C. R. Acad. Sci. Paris 232, 1803 (1951).

M 12, 840

Lévy, P., Systèmes markoviens et stationnaires, Cas dénombrable, Ann. Sci. École Norm. Sup. 68, 327 (1951).

M 13, 959

Lévy, P., Processus à la fois stationnaires et markoviens pour les systèmes ayant une infinité dénombrable d'états possibles, Proc. Int. Cong. Math., 1, 549 (Amer. Math. Soc., Providence 1952).

M 13, 567

Lévy, P., Complément à l'étude des processus de Markoff, Ann. Sci. École Norm. Sup. 69, 203 (1952).

M 14, 663 
Lévy, P., Processus markoviens et stationnaires du cinquième type (infinité dénombrable d'états possibles, paramètre continu), C.R.Acad. Sci. Paris 236, 1630 (1953).

M 15, 44

Lévy, P., Rectification au texte d'une note antérieure, C.R. Acad. Sci. Paris 23\%, 964 (1953). M 15, 44

Lévy, P., Rectification à un théorème sur le mouvement brownien à $p$ paramètres, $C$. R. Acad. Sci. Paris P38, 2140 (1954).

M 16, 150

Linnik, Y. V., On nonstationary Markov chains, Doklady Akad. Nauk SSSR 60, 21 (1948).

M 9, 520

Linnik, Y. V., On the theory of nonuniform Markov chains, Izvestiya Akad. Nauk SSSR. Ser. Mat. 13, $65(1949)$

M 10, 552

Linnik, Y. V., Multiple integrals and local laws for inhomogeneous Markov chains, Izvestiya Akad. Nauk SSSR Ser. Mat. 13, 533 (1949). M 11, 606

Manevič, D. V., On a local limit theorem for stationary Markov chains, Dokl. Akad. Nauk Üzbek. SSR. \%, 5 (1953).

M 1\%, 978

Maravall Casesnoves, D., Impulsive random motion and hereditary stochastic processes, Rev. Mat. Hisp.-Amer. 15, 9 (1955).

M 17, 867

Maruyama, G., Markov processes and stochastic equations, Nat. Sci. Rep. Ochanomizu Univ. 4, 40 (1953).

M 15, 448

Maruyama, G., On the transition probability functions of the Markov process, Nat. Sci. Rep. Ochanomizu Univ. 5, 10 (1954).

M 16, 724

Maruyama, G., Continuous Markov processes and stochastic equations, Rend. Circ. Mat. Palermo 4, 48 (1955)

M 1\%, 166

- Mathews, P. M., (See A. Ramakrishnan) Proc. Indian Acad. Sci. Sect. A. 38, 450 (1953).

Mihoc, G., Sur un théorème de limite, Bull. Math. Soc. Roum. Sci. 35, 173 (1933).

Z 8, 366

Mihoc G., Sur les chaînes multiples discontinues, C. R. Acad. Sci. Paris 198, 2135 (1934).

Mihoc, G., Über allgemeine Eigenschaften von voneinander abhängigen-statistischen Variablen, Bull. Math. Soc. Roum. Sci. 3\%, 37 (1935).

Z 14, 29

- Mihoc, G., (See O. Onicescu) Bull. Sci. Math., II. 59, 174 (1935).

- Mihoc, G., (See O. Onicescu) C. R. Acad. Sci., Paris 20\%, 2031 (1936).

- Mihoc, G., (See O. Onicescu) C. R. Acad. Sci., Paris 205, 481 (1937).

- Mihoc, G., (See O. Onicescu) Actual. Sci. Industr. $N r .503$ (Paris: Hermann \& Cie, 1937).

- Mihoc, G., (See O. Onicescu) Acad. Roum. Bull. Sect. Sci. 21, 110 (1939).

- Mihoc, G., (See O. Onicescu) Bull. Math. Soc. Roum. Sci. 41, 99 (1939).

- Mihoc, G., (See O. Onicescu) Acad. Roum. Bull. Sect. Sci. 22, 231 (1940).

-Mihoc, G., (See O. Onicescu) Mathematica, Cluj 16, $13(1940)$

- Mihoc, G., (See O. Onicescu) Acad. Roum. Études Recherches 14 (1943).
Mihoc, G., Sur le problème des itérations dans une suite d'épreuves, Bull. Math. Soc. Roumaine Sci. 45, 81 (1943).

M $\boldsymbol{g}, 19$

- Mihoc, G., (See O. Onicescu) Acad. Roum. Bull. Sect. Sci. 24, 401 (1943).

- Mihoc, G., (See O. Onicescu) Bull. Math. Soc. Roumaine Sci. 46, 77 (1944).

- Mihoc, G., (See O. Onicescu) Bull. Math. Soc. Roumaine Sci. 48, 32 (1947).

Mihoc, G., La loi des événements rares pour les chaînes de Markoff, Acad. Repub. Pop. Române. Mat. Fiz. 4, 783 (1952).

M 15, 635

Mihoc, G., La loi des événements rares pour les chaînes de Markov, Science R.P. Roumaine 1, 14 (1953).

M 17, 980

Mihoc, G., Markov chains, Gaz. Mat. Fiz. Ser. A. 6, 438 (1954).

M 16, 495

Mihoc, G., Extension de la loi de Poisson pour les chaînes de Markov, multiples et homogenes, Acad. Repub. Pop. Romîne. Bul. Mat. Fiz. 6, 5 (1954).

M 16, 724

Mihoc, G., Über verschiedene Ausdehnungen des Poissonschen Gesetzes auf endliche konstante Markoffsche Ketten, Ber. Wahrsch. Stat. (Verlag Wissenschaften, Berlin 1956).

M 18, 605

Milicer-Gruzewska, H., Un schéma probabiliste de processus stochastique, C.R. Acad. Sci. Paris $\mathbf{2 3 3}$, 1345 (1951).

M 13, 664

Montroll, E. W., On the theory of Markoff chains, Ann. Math. Statist. 18, 18 (1947). M 8, 472

Montroll, E. W., Markoff chains, Wiener integrals, and quantum theory, Comm. Pure Appl. Math. 5, 415 (1952).

M 14, 663

Moran, P. A. P., A class of complex Markoff chains, Quart. J. Math., Oxford Ser. 19, 140 (1948)

M 10, 132

Morimura, H., On a renewal theorem, Kōdai Math. Sem. Rep. 8, 125 (1956).

M 18, 942

Mou, T. C., Some mathematical considerations which play a role in diffusion problems, Nederl. Akad. Wetensch. Verslagen 53, 400 (1944). M 8,315

Moustafa, M. D., Infinite matrix-products associated with Markov chains, Indag. Math. 17, 234 (1955).

M 16, 893

Moustafa, M. D., Input-output Markov processes, Indag. Math. 19, 112 (1957).

M 18, 831

Münzner, H., Über eine spezielle Markoffsche Kette am Galtonbrett, Z. Angew. Math. Mech. 14, 343 (1934).

Z 10, 312

Nakamura, G., (See K. Udagawa) Kōdi Math. Sem. Rep. 8, 117 (1956).

Neveu, J., Semi-groupes généralisés et processus de Markoff, C. R. Acad. Sci. Paris 240, 1046 (1955).

M 1\%, 50

Neveu, J., Jeux de Markoff et problèmes d'absorption, C. R. Acad. Sci. Paris \$40, 2372 (1955)

M 1\%, 167

-Onicescu, O., Sur les chaînes de variables statistiques, Bull. Sci.Math., II. 59, 174 (1935). Z 12, 28

- Onicescu, O., Sur les chaînes statistiques, $C$. $R$. Acad. Sci. Paris 202, 2031 (1936).

$\mathrm{Z} \mathbf{1 4}, 223$ 
Onicescu, O., Sur la notion de chaîne et l'idée de loi naturelle, Mathematica, Cluj 13, 66 (1937).

Z 18, 156

Onicescu, O., L'allure asymptotique de la somme des variables d'une chaîne de Markoff discontinue, C. R. Acad. Sci., Paris 205, 481 (1937). Z 1\%, 124

-Onicescu, O., La dépendance statistique chaînes et familles de chaînes discontinues, Actual. Sci. et Industr. Nr. 503 (Paris: Hermann \& Cie. 1937).

Z 1\%, 272

Onicescu, O., Le principe ergodique et les probabilités en chaîne, Actual. Sci. et Indus. Nr. $\mathbf{g 3 g}^{3}$ (Paris: Hermann and Cie. 1938).

Z 2\%, 242

-Onicescu, O., Sur l'application des équations fonctionnelles de Chapman et Smoluchowsky dans la théorie des chaînes de Markoff, Acad. Roum. Bull. Sect. Sci. 21, 110 (1939)

M 1, 247

Onicescu, O., Sur les sommes de variables enchaînées, II, Bull. Math. Soc. Roum. Sci. 41, 99 (1939).

Z 21, 41

Onicescu, O., Propriétés asymptotiques des chaînes de Markoff étudiées à l'aide de la fonction caractéristique, Mathematica, Cluj 16, 13 (1940).

M 1, 342

Onicescu, O., Sur les sommes de variables enchaînées dans le cas d'un ensemble numérable de valeurs, Acad. Roum. Bull. Sect. Sci. ?2, 231 (1940).

M 1, 246

-Onicescu, O., Les chaînes de variables aléatoires, Problémes asymptotiques, Acad. Roum. Études. Recherches 14, 167 pages (1943).

M 10, 311

Onicescu, O., Un cas d'exception dans la théorie des chaînes de Markoff, Acad. Roum. Bull. Sect. Sci. 24, 401 (1943).

M 9, 520

- Onicescu, O., Le coefficient de dispersion et la dépendance des épreuves, Bull. Math. Soc. Roumaine Sci. 46, 77 (1944).

M \%, 459

Onicescu, O., Chaînes de mouvements discontinus et changements d'état, Bull. Math. Soc. Roumaine Sci. 48, 32 (1947).

M 11, 257

Opatowski, I., An inverse problem concerning a chain process, Proc. Nat. Acad. Sci. U.S.A. 28, 83 (1942).

M 4, 17

Opatowski, I., Markoff chains with reverse transitions, Proc. Nat. Acad. Sci. U.S.A. 31, 411 (1945).

M $\%, 210$

Orts, J. M., On certain iterated probabilities, Revista Mat. Hisp.-Amer. 4, 153 (1944).

M 6, 233

○shiro, S., (See J. Fennell), Naval Res. Logist. Quart. 3, 19 (1956).

Ottaviani, G., Sulle catene doppie di Markoff, Giorn. 1st. Ital. Attuari 14, 7 (1952). M 15, 330

Pal, L., On the theory of stochastic processes in cosmic radiation, Z̆. Eksper, Teoret, Fiz. 30, 362 (1956).

M 18, 76

Patankar, V. N., A note on recurrent events, Proc. Cambridge Philos. Soc. 51, 96 (1955). M 16, 494

Persidskij, K., Über das Markoffsche Theorem, Izv. Fiz. Mat. Obsc. Kazan. Univ. 4, 37 (1931).

Z 2, 406
Popoff, C., Osservazioni sulla teorie delle probabilità concatenate di Markoff, Univ. Roma e Ist. Naz. Alta Mat. Rend. Mat. e Appl. 3, 282 (1942).

M 8, 281

Popoff, K., Observations sur la théorie des probabilitiés en chaîne de Markoff. Cas d'une suite continue d'épreuves, Annuaire U. Sofia. Fac. Phys.-Math. Livre 1, 38, 319 (1942). M 1\%, 269

Potoček, J., Sur la dispersion dans la théorie des chaînes de Markoff, Publ. Fac. Sci. Univ. Masaryk Nr. 154, 1 (1932)

Z 5, 255

Potočk, J., Une reinarque sur les chaînes de Markoff réversibles, C. R. Acad. Sci. Paris 206, 1536 (1938).

Z 18, 414

Quenouille, M. H., On the problem of random flights, Proc. Cambridge Philos. Soc. 43, 581 (1947).

М 9, 96

Ramakrishnan, A., Some simple stochastic processes, J. Roy. Statist. Soc. Ser. B. 13, 131 (1951).

M 13, 664

Ramakrishnan, A., On a class of stochastic integrodifferential equations, Proc. Indian Acad. Sci. Sect. A. 38, 450 (1953).

M 15, 723

Ramakrishnan, A., Inverse probability and evolutionary Markoff stochastic processes, Proc. Indian Acad. Sci. Sect. A. 41, 145 (1955). M 16, 1130

Ramakrishnan, A., Phenomenological interpretation of the integrals of a class of random functions, I, II, Nederl. Akad. Wetensch. Proc. Ser. A. 58, 470 (1955).

M 18, 637

Ramakrishnan, A., Processes represented as integrals of a class of random functions, Nederl. Akad. Wetensch. Proc. Ser. A. 59, 120 (1956). M 1\%, 865 Reuter, G. E. H., On the differentiable equations for the transition probabilities of Markov processes. with enumerably many states, Proc. Cambridge Philos. Soc. 49, 247 (1953). M 14, 757

Reuter, G. E. H., A note on contraction semigroups, Math. Scand. 3, 275 (1956).

M 1\%, 988

Robbins, H. (See T. E. Harris) Proc. Nat. Acad. Sci. U.S.A. 39, 860 (1953).

Rodeanu, R., Sur un théorème ergodique concernant les chaînes de Markov, Rev. Univ. Politehn. Bucuresti. Ser. Sti. Nat. 4, 39 (1955). M 1\%, 980

Romanovskiŭ, V. I., Diskretnye Cepi Markova, ('Tehniko-Teoretičeskoľ Literatury, Moscow 1949).

M 11, 445

Romanovsky, V., Recherches sur les chaînes de Markoff, I, mém, Acta Math. 66, 147 (1936).

Z 14, 28

Romanovsky, V., Le principe ergodique et les probabilités en châ̂ne (Paris: Hermann and Cie. 1938).

Z 2\%, 242

Rosenblatt, M., On a class of Markov processes, Trans. Amer. Math. Soc. 81, 120 (1951). M 13, 258

Rutman, M. A., Concerning a paper by T. A. Sarymsakov, C. R. Acad. Sci. URSS 52, 567 (1946).

M 8, 215

$\checkmark$ Saleme, E. (See F. Cernuschi) An. Soc. Ci. Argentina 138, 201 (1944). 
Sapogov, N. A., On singular Markov chains, Doklady Akad. Nauk SSSR 58, 193 (1947). M 9, 293

Sapogov, N. A., The Laplace-Lyapunov limit theorem for singular Markov chains, Doklady Akad. Nauk SSSR 58, 1905 (1947).

M 9, 361

Sapogov, N. A., On the law of the iterated logarithm for Markov chains, Uspehi Matem. Nauk 4, 195 (1949).

M 11, 189

Sapogov, N. A., A two-dimensional limit theorem for two-dimensional chains, Izvestiya Akad. Nauk SSSR. Ser. Mat. 13, 301 (1949).

M 11, 40

- Sapogov, N. A. (See Y. V. Linnik) Izvestiya Akad. Nauk SSSR. Ser. Mat. 13, 533 (1949).

Sapogov, N. A., On multidimensional inhomogeneous Markov chains, Doklady Akad. Nauk SSSR 69, 133 (1949).

M 11, 256

Sarmanov, O. V., Necessary and sufficient conditions of existence of a discrete limit law for Markov chains with two states, Dokl. Akad. Nauk SSSR. 110, 735 (1956).

M 18, 832

Sarymsakov, T. A., The law of the iterated logarithm for Markov schemes, Acta [Trudy] Univ. Asiae Mediae. Math. Fasc. 5, 15 pp (1945). M 8, 522

Sarymsakov, T. A., Sur les chaînes de Markoff à une infinité dénombrable d'états possibles, $C . R$. (Doklady) Acad. Sci. URSS 4\%, 617 (1945).

$\mathrm{M} \boldsymbol{\gamma}, 314$

Sarymsakov, T. A., Sur une synthèse des deux méthodes d'exposer la théorie des chaînes discrètes de Markoff, C. R. (Doklady) Acad. Sci. URSS 48, 159 (1945).

M $\boldsymbol{7}, 459$

Sarymsakov, T. A., Un nouveau critère nécessaire et suffisant pour la régularité des chaînes de Markoft dont l'ensemble des états possibles est continu. C. R. (Dcklady) Acad. Sci. URSS 49, 85 (1945).

$\mathrm{M} \boldsymbol{\gamma}, 459$

- Sarymsakov, T. A., The law of the iterated logarithm for Markov chains, Doklady Akad. Nauk SSSR 59, 1249 (1948).

M 9, 451

Sarymsakov, T. A., On a property of the characteristic numbers of an integral-equation with a nonnegative and continuous kernel, Doklady Akad. Nauk SSSR 6\%, 973 (1949).

M 11, 184

Sarymsakov, T. A., On the ergodic principle for nonstationary Markoff chains, Doklady Akad. Nauk SSSR 90, 25 (1953).

M 15, 45

Sarymsakov, T. A., Osnovy teorii processov Markova (Elements of the theory of Markov processes) (Gosudarstv. Izdat. Tehn.-Teor. Lit., Moscow 1954).

M 16, 1129

- Scheffer, C. (See D. van Dantzig) Nederl. Akad. Wetensch. Proc. Ser. A 5\%, 377 (1954).

Schulz, G., Über Markoffsche Ketten höherer Ordnung, Z. Angew, Math. Mech. 13, 235 (1933).

$\mathrm{Z} \%, 252$

Schulz, G., Über die Häufigkeit der Iterationen in einer Beobachtungfolge, Deutsche Math. $\%, 22$ (1942).

M 8, 215

- Siegert, A. J. F. (See D. A. Darling) Ann. Math. Statist. 24, 624 (1953).

- Siegert, A. J. F. (See D. A. Darling) Proc. Nat. Acad. Sci. U.S.A. 42, 525 (1956).
Silvey, S. D., A problem associated with a particular Markov chain, Proc. Glasgow Math. Assoc. 2, 100 (1954).

M 16, 379

Siraždinov, S. H., Limit theorems for stationary Markor chains, (Izdat. Akad. Nauk Uzbekskoi. SSR, Taškent, 1955).

M 18, 944

Sirokorad, B. V., On the applicability of the central limit theorem to Markov chains, Izvestiya Akad. Nauk SSSR. Ser. Mat. 18, 95 (1954). M 15, 635

Smirnov, N. V., On the statistical estimation of transition probabilities in Markov chains, Vestnik Leningrad. Univ. 10, 47 (1955). $\quad$ M 18, 757

- Smith, R. T. (See H. B. Horton), Ann. Math. Statist. 20, 82 (1949).

$\checkmark$ Snell, J. L. (See J. G. Kemeny) (Prentice Hall, Englewood Cliff's, N.J., 1957).

Soulé, J. L., Energie cinétique moyenne d'une particule en mouvement brownien dans un fluide réel, Publ. Inst. Statist. Univ. Paris 4, 93 (1955).

M 1\%, 51

- Sultanova, M. (See T. A. Sarymsakov), Doklady Akad. Nauk SSSR 59, 1249 (1948).

Takács, L., On a probability problem arising in the theory of counters, Proc. Cambridge Philos. Soc. 52, 488 (1956).

M 18, 424

Taylor, S. J., The $\alpha$-dimensional measure of the graph and set of zeros of a Brownian path, Proc. Cambridge Philos. Soc. 51, 265 (1955). M 1\%, 595

Tchen, C. M., Mean Value and Correlation Problems Connected with the Motion of Small Particles Suspended in a Turbulent Fluid (Martinus Nijhoff, The Hague, 1947).

M 9, 47

Theodorescu, R., Processus stochastiques de multiplicité p, Acad. R. P. Romîne. Bul. Sti. Sect. Sti. Mat. Fiz. \%, 775 (1955).

M 1\%, 636

Theodorescu, R., Sur les relations caractéristiques des chaînes de Markoff continues de multiplicité p, Acad. R. P. Romîne. Bul. Sti. Sect. Sti. Mat. Fiz. \%, 763 (1955).

M 1\%, 635

Theodorescu, R., Chaînes continues à liaisons complétes de multiplicité p, Com. Acad. R. P. Romîne. 6, 253 (1956).

M 18, 157

Thompson, G. L. (See J. G. Kemeny) (Prentice Hall, Englewood Cliffs, N.J., 1957).

Tortrat, A., Sur les fonctions de corrélation des processus de Markoff, C. R. Acad. Sci. Paris 228, 1559 (1949).

M 10, 720

Udagawa, K., On a certain queuing system, Kōdai Math. Sem. Rep. 8, 117 (1956).

M 18, 770

Urbanik, K., Limit properties of homogeneous Markoff processes with a denumerable set of states, Bull. Acad. Polon. Sci. Cl. III. R, 371 (1954).

M 16, 601

Vescan, T., Introduction à l'étude statistique des équations du mouvement des fluides, Bull. Sci. École Polytech. Timişoara 9, 287 (1940). M 9,520

- Vigier, J. P. (See A. Fuchs), C. R. Acad. Sci. Paris. 242, 1120 (1956). 
Wang, S-J., On the differentiabilities of the transition probabilities in temporally-homogeneous Markoff process with enumerable number of states, Acta Math. Sinica. 4, 359 (1954). M 18, 424

Watanabe, Y., Aufgaben betreffend das Irrfahrtproblem, J. Gakugei Tokushima Univ. Nat. Sci. Math. 6, 41 (1955).

M 1\%, 1097

Whittle, P., Some distribution and moment formulae for the Markov chain, J. Roy. Statist. Soc. Ser. B. 1\%, 235 (1955).

M 17, 982

Wolfowitz, J., Remarks on the notion of recurrence, Bull. Amer. Math. Soc. 55, 394 (1949). M 10, 549

Yaglom, A. M., The ergodic principle for Markov processes with stationary distributions, Doklady Akad. Nauk SSSR 56, 347 (1947).

M 9, 149

Yaglom, A. M., On the statistical treatment of Brownian motion, Doklady Akad. Nauk SSSR 56, 691 (1947).

M 9, 150

Yaglom, A. M., Über die statistische Umkehrbarkeit der Brownschen Bewegung (Verlag Kultur und Fortschritt, Berlin, 1953).

M 1\%, 51

Yosida, K., Operator-theoretical treatment of the Markoff's process, Proc. Imp. Acad. Jap. 14, 363 (1938)

$\mathrm{Z} \mathbf{2 0}, 146$

Yosida, K., Operator-theoretical treatment of Markoff's process, II, Proc. Imp. Acad. Jap. 15, 127 (1939).

Z 21，422

Yosida, K., Markoff process with an enumerable infinite number of possible states, Jap. J. Math. 16, 47 (1939).

M 1, 62

Yosida, K., The Markoff process with a stable distribution, Proc. Imp. Acad. Toyko 16, 43 (1940).

M 1, 343

- Yosida, K., Operator-theoretical treatment of Markoff's process and mean ergodic theorem, Ann. of Math. 4R, 188 (1941). M 2230

Yosida, K., Simple Markoff process with a locally compact phase space, Math. Japonicae 1, 99 (1948).

M 11, 41

Yosida, K., An operator-theoretical treatment of temporally homogeneous Markoff process, $J$. Math. Soc. Japan 1, 244 (1949).

M 12, 190

Yosida, K., Brownian motion on the surface of the 3sphere, Ann. Math. Statist. 20, 292 (1949).

M 10, 721

Yosida, K., On the generating parametrix of the stochastic processes, Proc. Nat. Acad. Sci. U.S.A. 41, 240 (1955).

$\mathrm{M} \mathbf{1 \%}, 167$

\section{Stationary Processes}

Austin, D. G., On the existence of the derivative of Markoff transition probability functions, Proc. Nat. Acad.Sci.U.S.A.41, 224 (1955). M 16, 1130

Bartlett, M. S., On the theoretical specification and sampling properties of autocorrelated time series, Suppl. J. Roy. Statist. Soc. 8, 27 (1946).

M 8, 283

Bartlett, M. S., Periodogram analysis and continuous spectra, Biometrika 3\%, 1 (1950).

M 12, 35
Bartlett, M. S., The statistical analysis of stochastic processes, Colloq. Statist. Bruxelles (Masson \& Cie., Paris 1955).

M 1\%, 506

Batchelor, G. K., Diffusion in a field of homogeneous turbulence II. The relative motion of particles, Proc. Cambridge Philos. Soc. 48, 345 (1952).

M 13, 879

Bayley, G. V., The "effective" number of independent observations in an autocorrelated time series, Suppl. J. Roy. Statist. Soc. 8, 184 (1946).

M 8, 591

-Blanc-Lapierre, A., Les fonctions aléatoires stationnaires et la loi des grands nombres, Bull. Soc. Math. France $\mathbf{7 4 ,} 102$ (1946).

M 9, 293

Blanc-Lapierre, A., Propagation d'une perturbation à spectre peu étendu dans un milieu dispersif non absorbant, Revue Sci. 84, 579 (1946).

M 8, 472

-Blanc-Lapierre, A., Sur la décomposition spectrale des fonctions aléatoires stationnaires d'ordre deux, C. R. Acad. Sci. Paris 222, 467 (1946). M \%, 460

-Blanc-Lapierre, A., Résultats sur la décomposition spectrale des fonctions aléatoires stationnaires d'ordre 2, C. R. Acad. Sci. Paris 22\%, 713 (1946).

$\mathrm{M} \boldsymbol{\gamma}, 460$

Blanc-Lapierre, A., Sur la structure des fonctions aléatoires strictement stationnaires à spectre totalement discontinu, C.R. Acad. Sci. Paris \%2\%, 1155 (1946).

M 8, 38

Blanc-Lapierre, A., Extension de la méthode des filtres à des fonctions aléatoires non stationnaires, C.R. Acad. Sci. Paris 22\%, 1270 (1946). M \%, 460

-Blanc-Lapierre, A., Les fonctions aléatoires stationnaires de plusieurs variables, Revue Sci. 85, 419 (1947).

M 9, 150

-Blanc-Lapierre, A., Sur une propriété fondamentale des fonctions de corrélation, C.R. Acad. Sci. Paris 224, 786 (1947).

M 8, 472

Blanc-Lapierre, A., Analyse harmonique des fonctions aléatoires stationnaires, Colloq. Inter. Centre Nat. de la Rech. Sci., 15, Paris, (1949). M 11, 257

Blanc-Lapierre, A., Remarques sur un théorème d'interpolation, C. R. Acad. Sci. Paris 234, 1733 (1952).

M 13, 958

Blum, J. R., A class of stationary processes and a central limit theorem, Proc. Nat. Acad. Sci. U.S.A. 42, 412 (1956).

M 18, 342

- Blum, J. R., A class of stationary processes and a central limit theorem, Duke Math. J. 24, 73 (1957).

M 18, 680

Bochner, S., Harmonic analysis and the theory of probability (Univ. Calif. Press, Berkeley and L.A., 1955).

M 1\%, 273

Bochner, S., Sturm-Liouville and heat equations whose eigenfunctions are ultraspherical polynomials or associated Bessel functions, Proc. Conf. Diff. Eq. (Univ. Maryland, 1956).

M 18, 484

Bochner, S., Stationarity, boundedness, almost periodicity of random-valued functions, Proc. Symp. Stat. Prob., II, 7 (1956).

M 18, 940

- Bode, H. W., A simplified derivation of linear least square smoothing and prediction theory, Proc. I.R.E. 38, 417 (1950).

M 11, 672 
-Brard, R., (See Blanc-Lapierre) Bull. Soc. Math. France $\mathbf{~ 4 , ~} 102$ (1946).

Burgers, J. M., Spectral analysis of an irregular function, Nederl. Akad. Wetensch. Proc. 51, 1073 (1948).

M 10, 311

Cramér, H., On the theory of stationary random processes, Ann. of Math. 41, 215 (1940). M 1, 150

Davis, R. C., On the Fourier expansion of stationary random processes, Proc. Amer. Math. Soc. 4, 564 (1953).

M 15, 45

Dedebant, G., On a new definition of random function and its ergodic theorem, Symp. Mathmáticos, Montevideo, Uruguay (1954).

M 1\%, 49

Diederich, F. W., The dynamic response of a large airplane to continuous random atmospheric dis. turbances, J. Aero. Sci. 23, 917 (1956). M 18, 439

Dobrusin, R. L., On conditions of regularity of stationary Markov processes with a denumerable number of possible states, Uspehi Matem. Nauk \%, 185 (1952).

M 14, 567

-Fortet, R., (See Blanc-Lapierre) C.R. Acad. Sci. Paris 2\%2, 467 (1946).

- Fortet, R., (See Blanc-Lapierre) C.R. Acad. Sci. Paris 222, 713 (1946).

- Fortet, R., (See Blanc-Lapierre) C. R. Acad. Sci. Paris 222, 1155 (1946).

- Fortet, R., (See Blanc-Lapierre) C. R. Acad. Sci. Paris 222, 1270 (1946).

-Fortet, R., (See Blanc-Lapierre) Revue Sci. 85, 419 (1947).

- Fortet, R., (See Blanc-Lapierre) C. R. Acad. Sci. Paris 224, 786 (1947).

Franckx, E., Chaînes de Markoff et échelles numériques, Trabajos Estadistica 1, 147 (1950).

M 13, 258

-Freund, J. E., (See I. Miller) J. Roy. Statist. Soc. Ser. B. 18, 257 (1956).

Grenander, U., On empirical spectral analysis of stochastic processes, Ark. Mat. 1, 503 (1952).

M 14, 187

Grenander, U., On Toeplitz forms and stationary processes, Ark. Mat. 1, 555 (1952). M 14, 187

- Grenander, U., On spectral analysis of stationary time series, Proc. Nat. Acad. Sci. U.S.A. 38, 519 (1952).

M 14, 61

- Grenander, U., Statistical spectral analysis of time series arising from stationary stochastic processes, Ann. Math. Statist. 24, 537 (1953).

M 15, 448

Hájek, J., Linear estimation of the mean value of a stationary random process with convex correlation function, Czechoslovak Math. J. 6, 94 (1956).

M 18, 241

- Hammersley, J. M., (See G. V. Bayley) Suppl. J. Roy. Statist. Soc. 8, 184 (1946).

Hanner, O., Deterministic and non-deterministic stationary random processes, Ark. Mat. 1, 161 (1950).

M 11, 606
Heine, V., Models for two-dimensional stationary stochastic processes, Biometrika 42, 170 (1955).

M 1\%, 167

Ionescu Tulcea, C. 'T., Un théorème ergodique, Com. Acad.R. P. Romîne. 1, 23 (1951). M 1\%, 49

Itô, H., Observed value of the autocorrelation function, Proc. Japan Acad. 29, 198 (1953).

M 15, 330

Itô, H., The observation theory of the stationary random process, Proc. Japan Acad. 29, 305 (1953). M 15, 969

Itô, K., A kinematic theory of turbulence, Proc. Imp. Acad. Tokyo 20, 120 (1944).

M \%, 313

Itô, K., On the ergodicity of a certain stationary process, Proc. Imp. Acad. Tokyo 20, 54 (1944).

M 7,313

Itô, K., On the normal stationary process with no hysteresis, Proc. Imp. Acad. Tokyo 20, 199 (1944).

М $\%, 312$

Itô, K., Stationary random distributions, Mem. Coll. Sci. Univ. Kyoto. Ser. A. Math. 28, 209 (1954).

M 16, 378

Kac, M., An explicit representation of a stationary Gaussian process, Ann. Math. Statist. 18, 438 (1947).

M 9, 97

Kac, M., On the notion of recurrence in discrete stochastic processes, Bull. Amer. Math. Soc. 53, $1002(1947)$.

M 9, 194

Kampé de Fériet, J., Fonctions aléatoires définies sur un groupe abstrait, C.R. Acad. Sci. Paris $\mathbf{2 2 5}$, 428 (1947).

M 9, 97

Kampé de Fériet, J., Analyse harmonique des fonctions aléatoires strictement stationnaires, $C . R$. Acad. Sci. Paris 225, 623 (1947). M 9, 150

Kampé de Fériet, J., Analyse harmonique des fonctions aléatoires stationnaires d'ordre 2 définies sur un groupe abélien localement compact, C.R. Acad. Sci. Paris 226, 868 (1948).

M 9, 520

Kampé de Fériet, J., Fonctions aléatoires stationnaires et groupes de transformations dans un espace abstrait, Colloques Intern. Centre Nat. Rech. Sci. 13, 67 (Paris, 1949). M 11, 258

Kanô, S., On the prediction problem of a stationary stochastic process, Mem. Fac. Sci. Kyūsyū Univ. A. 6, $173(1952)$.

M 14, 889

Karhunen, K., Lineare Transformationen stationärer stochastischer Prozesse, C. R. Cong. Math. Scandinaves p. 320 (J. G. Forlag, Copenhagen, 1947).

M 8, 391

Karhunen, K., Zur Interpolation von stationären zufälligen Funktionen, Ann. Acad. Sci. Fennicae. Ser. A. I. Math. Phys. No. 142, (1952). M 14, 772 Kawata, T., Stationary process and harmonic analysis, Kōdai Math. Sem. Rep. 1953, 41 (1953).

M 15, 45

Kawata, T., Remarks on prediction problem in the theory of stationary stochastic processes, Tôhoku Math. J. 6, 13 (1954).

M 16, 151

Kendall, D. G., Some analytical properties of continuous stationary Markov transition functions, Trans. Amer. Math. Soc. 78, 529 (1955).

M 16, 725 
Kendall, M. G., Contributions to the Study of Oscillatory Time-Series, (Cambridge, Univ. Press or N.Y., Macmillan Co., 1946).

M 8, 471

Khintchine, A., Mathematical methods of the theory of mass service. (Matematičeskie metody teorii massovogo obsluživaniya) Trudy Mat. Inst. Steklov. 49, (1955).

M 1\%, 276

Kolmogoroff, A., Sur l'interpolation et extrapolation des suites stationnaires, $C$. R. Acad. Sci., Paris 208, 2043 (1939).

Z 21,422

Kolmogoroff, A., Interpolation und extrapolation von stationären zufälligen folgen, Bull. Acad. Sci. URSS. Sér. Math. 5, 3 (1941).

M 3, 4

Kolmogoroff, A., Stationary sequences in Hilbert spaces, Trabajos Estadistica 4, 55 (1953).

M 15, 449

Kolmogoroff, A., Stationary sequences in Hilbert's space Bolletin Moskovskogo Gosudar. U. Matematika 2 (1941).

M 5, 101

Kosulajeff, P. A., Sur les problèmes d'interpolation et d'extrapolation des suites stationnaires, $C$. $R$. Acad. Sci. URSS 30, 13 (1941).

M 3, 4

Kozulyaev, P. A., On a question of extrapolation of stationary random processes, Doklady Akad. Nauk SSSR 56, 903 (1947).

M 9, 149

Lapostolle, P., (See Blanc-Lapierre) Revue Sci.84, 579 (1946).

Lévy, P., L'analyse harmonique des fonctions aléatoires stationnaires. Colloq. Intern. Centre Nat. Rech. Sci. No. 15, 111, Paris (1949).

M 11, 376

Lévy, P., Eléments de la théorie des processus à la fois stationnaires et de Markoff, dans le cas d'un système ayant une infinité dénombrable d'états possibles, C. R. Acad. Sci. Paris, 231, 467 (1950).

M 12, 269

Linnik, Yu. V., On a question of the statistics of dependent events, Izxestiya Akad. Nauk SSSR. Ser. Mat. 14, 501 (1950).

M 12, 512

Maruyama, G., The harmonic analysis of stationary stochastic processes, Mem. Fac. Sci. Kyūsyū Univ. A., 4, 45 (1949).

M 11, 257

- Mihoc, G., (See O. Onicescu). Disquisit. Math. Phys. 1, $61(1940)$.

- Miller, I., Expected arc length of a Gaussian process on a finite interval, J. Roy Statist. Soc. Ser. B. 18, 257 (1956).

M 18, 681

Moran, P. A. P., The spectral theory of discrete stochastic processes, Biometrika 36, 63 (1949).

M 11, 606

Onicescu, O., Comportement asymptotique des chaînes à liaisons complètes, Disquisit, Math. Phys. 1, 61 (1940).

M 8, 591

Onoyama, T., Random frequency process, Bull. Math. Statist. 5, 51 (1952).

M 14, 663

Palmer, D. A., Properties of random functions, Prcc. Cambridge Philos. Soc. 52, 672 (1956).
Palmer, D. A., Corrigenda to the paper "Properties of random functions," Proc. Cambridge Philos. Soc. 53, 266 (1957).

M 18, 681

- Pinsker, M. S., On linear extrapolation of random processes with stationary $\mathrm{n}^{\text {th }}$ increments, Deklady Akad. Nauk SSSR 94, 385 (1954). M 15, 806

$\checkmark$ Pitcher, T. S., (See W. L. Root), Ann. Math. Statist. 26, 313 (1955).

Quenouille, M. H., Notes on the calculation of autocorrelations of linear autoregressive schemes, Biometrika, 34, 365 (1947).

M 9, 361

Raevskiľ, S. Y., On some typical nonlinearities for continuous random events, Vestnik Moskov. Univ. 10, 37 (1956).

$\mathrm{M} \mathbf{1 \%}, 755$

Roberts, J. D., A theory of biased dice, Eureka. 18, 8 (1955).

M 1\%, 274

$\checkmark$ Root, W. L., On the Fourier series expansion of random functions, Ann. Math. Statist. 26, 313 (1955).

M 17, 49

-Rosenblatt, M., (See U. Grenander), Proc. Nat. Acad. Sci. U.S.A. 38, 519 (1952).

Rosenblatt, M., (See U. Grenander) Ann. Math. Statist. 24, 537 (1953).

Rosenblatt, M., Some purely deterministic processes, (Research Div. College of Eng. N. Y. Univ., N. Y. 1956).

M 18, 241

Rosenblatt, M., (See J. R. Blum), Proc. Nat. Acat. Sci. U.S.A. 42, 412 (1956).

$\checkmark$ Rosenblatt, M., (See J. R. Blum), Duke Math. J. 24, 73 (1957).

Sarymsakov, T. A., On the theory of stationary stochastic processes without after effect (Differential equations for characteristic functions), Akad. Nauk Uzbek. SSR. Trudy Inst. Mat. Meh. 5, 61 (1949).

M 16, 495

$\checkmark$ Shannon, C. E., (See H. W. Bode), Proc. I.R.E. 38, 417 (1950).

$\checkmark$ Siegert, A. J. F., (See M. Kac) Ann. Math. Statist. 18, 438 (1947).

Skorohod, A. V., On the limiting transition from a sequence of sums of independent random quantities to homogeneous random process with independent increments. Dokl. Akad. Nauk SSSR. 104, 364 (1955).

M 1\%, 1096

Tortrat, A., Les processus strictement stationnaires de Markoff et leurs corrélations, J. Math. Pures Appl. 32, 281 (1953).

M 15, 449

Wold, H., On prediction in stationary time series, Ann. Math. Statist. 19, 558 (1948). $\quad$ M 10, 311

Wold, H., On stationary point processes and Markov chains, Skand. Aktuarietidskr. 31, 229 (1948).

M 10, 464

Wold, H., Sur les processus stationnaires ponctuels, Colloq. Int. Centre Nat. Rech. Sci. 13, 75, Paris (1949).

M 11, 258

Wold, H., Stationary time series, Trabajos Estadistica, $\mathbf{2 ,} 3$ (1951). 
Yaglom, A. M., On problems about the linear interpolation of stationary random sequences and processes, Uspehi Matem. Nauk 4, 173 (1949).

M 11, 119

Yaglom, A. M., Introduction to the theory of stationary random functions, Uspehi Matem. Nauk \%, 3 (1952).

M 14, 485

- Yaglom, A. M. (See M. S. Pinsker), Doklady Akad. Nauk SSSR 94, 385 (1954).

Yaglom, A. M., Effective solutions of linear approximation problems for processes with random stationary $\mathrm{n}^{\text {th }}$ increments, Doklady Akad. Nauk SSSR 98, 189 (1954).

M 16, 494

Yaglom, A. M., Theory of extrapolation and filtering of random processes, Ukrain. Mat. Ž 6, 43 (1954).

M 1\%, 167

Yaglom, A. M., Extrapolation, interpolation and filtering of stationary random processes with rational spectral density, Trudy Moskov. Mat. Obšč. 4, 333 (1955).

M 1\%, 167

Yaglom, A. M., Correlation theory of processes with random stationary $\mathrm{n}^{\text {th }}$ increments, Mat. $S b$. 3\%, 141 (1955).

M 1\%, 167

Yosida, K., Brownian motion on the surface of the 3-sphere, Ann. Math. Statist. 20, 292 (1949).

M 10, 721

Zasuhin, V., On the theory of multidimensional stationary random processes, $C . R$. (Doklady) Acad. Sci. URSS 33, 435 (1941). M 5, 102

\section{Special Processes}

Aitchison, J., On the distribution of a positive random variable having a discrete probability mass at the origin, J. Amer. Statist. Assoc. 50, 901 (1955).

M 1\%, 169

Ambarzumian, G. A., Study of a special case of a continuous stochastic process, Leningrad State Univ. Ann. 10, 120 (1940).

M 2,230

Ambarzumian, V. A., On the theory of brightness fluctuations in the Milky Way, C. R. Acad. Sci. URSS 44, 223 (1944).

M 6, 245

Ammeter, H., Die Elemente der kollektiven Risikotheorie von festen und zufallsartig schwankenden Grundwahrscheinlichkeiten, Mitt. Verein. Schweiz. Versich. Math. 49, 35 (1949).

M 10, 721

Anzai, H., A remark on spectral measures of the flow of Brownian motion, Osaka Math. J. 1, 95 (1949).

M 11, 118

Arley, N., On the elementary, time-homogeneous, discontinuous, stochastically definite process, Skand. Aktuarietidskr. 2\%, 172 (1944). M $\%, 210$

Bailey, N. T. J., A continuous time treatment of a simple queue using generating functions. J. Roy. Statist. Soc. Ser. B. 16, 288 (1954). M 16, 1035

Bartlett, M. S., The large-sample theory of sequential tests, Proc. Cambridge Philos. Soc. 42, 239 (1946).

Bartlett, M. S., Some evolutionary stochastic processes, J. Roy. Statist. Soc. Ser. B. 11, 211 (1949).

M 11, 672
Bartlett, M. S., The dual recurrence relation for multiplicative processes, Proc. Cambridge Philos. Soc. 4\%, 821 (1951).

M 13, 364

Bass, J., Les fonctions aléatoires et leur interprétation mécanique, Revue Sci. 83, 3 (1945).

Bass, J., Quelques conséquences mécaniques de l'équation de structure d'un corpuscule aléatoire, C. R. Acad. Sci. Paris 200, 272 (1945). M \%, 130

Bass, J., Sur la structure des fonctions aléatoires, C. R. Acad. Sci. Paris 220, 190 (1945). M \%, 130

Bass, J., Sur les fonctionnelles caractéristiques des solutions aléatoires de certaines équations aux dérivées partielles, C. R. Acad. Sci. Paris 238, $561(1954)$.

M 15, 969

-Bellman, R., On the theory of age-dependent stochastic branching processes, Proc. Nat. Acad. Sci. U.S.A. 34, 601 (1948). $\quad$ M 10, 311

- Bellman, R., On age-dependent binary branching processes, Ann. of Math. 55, 280 (1952).

M 13, 664

Birkhoff, G. D., What is the ergodic theorem?, Amer. Math. Monthly 49, 222 (1942). M 4, 15

- Blackwell, D., On functions of sequences of independent chance vectors with applications to the problem of the "random walk" in $\mathrm{k}$ dimensions, Ann. Math. Statist. 1\%, 310 (1946). M 8, 215

Blackwell, D., Controlled random walks, Proc. Int. Cong. Math. (Noordhoff, Groningen, 1956).

M 18, 979

Blanc-Lapierre, A., Remarques sur certaines fonctions aléatoires, C. R. Acad. Sci. Paris 22\%, 1333 (1948).

M 10, 385

Blanc-Lapierre, A., Quelques modèles statistiques utiles pour l'étude du bruit de fond, $C . R$. Acad. Sci. Paris 231, 566 (1950).

M 12, 270

Blanc-Lapierre, A., Remarques sur les fonctions aléatoires stationnaires laplaciennes, $C$. R. Acad. Sci. Paris 232, 934 (1951).

M 12, 620

Blanc-Lapierre, A., Sur l'analyse harmonique des fonctions aléatoires stationnaires, laplaciennes, C. R. Acad. Sci. Paris 232, 1070 (1951).

M 12, 620

Blanc-Lapierre, A., Considerations sur certains processus ponctuels et sur des fonctions aléatoires associées, Colloque Statist., Bruxelles (Masson \& Cie., Paris, 1955).

M 1\%, 502

Bochner, S., Fourier transforms of time series, Proc. Nat. Acad. Sci. U.S.A. 39, 302 (1953).

M 14, 1101

Bochner, S., Length of random paths on general homogeneous spaces, Ann. of Math. 5\%, 309 (1953).

M 14, 886

-Bogoliuboff, N., (See N. Kryloff), Ann. Chaire Phys. Math., Kiev 4, 243 (1939).

Bogolyubov, N., O Nekotoryh Statističeskih Metodah $v$ Matematiceskou Fizike. (On Some Statistical Methods in Mathematical Physics), (Akademiya Nauk Ukrainskoı̆ SSR, 1945).

M 8, 37

Borovickiı̌, S. I., On fluctuations in a linear system with periodically varying parameters, Doklady Akad. Nauk SSSR $\mathbf{7 4 ,} 233$ (1950).

M 12, 270 
Brigham, G., On a congestion problem in an aircraft factory, J. Operations Res. Soc. Amer. 3, 412 (1955).

M 1\%, 379

Burke, P. J., The output of a queuing system, $J$. Operations Res. Soc. Amer. 4, 699 (1956).

M 18, 707

-Cartwright, D. E., The statistical distribution of the maxima of a random function, Proc. Roy. Soc. London A $\mathbf{2 3 \%}, 212$ (1956).

M 18, 520

Čencov, N. N., Wiener random fields depending on several parameters, Dokl. Akad. Nauk SSSR. 106, 607 (1956).

M 1\%, 1101

Chandrasekhar, S., New methods in stellar dynamics, Ann. New York Acad. Sci. 45, 133 (1943).

M 5, 191

Chandrasekhar, S., The statistics of the gravitational field arising from a random distribution of stars. III. The correlations in the forces acting at two points separated by a finite distance, Astrophys. J. 99, 25 (1944).

M 5, 191

-Chung, K. L., Non-recurrent random walks, Pacific J. Math. 6, 441 (1956). $\quad$ M 18, 424

Consael, R., Sur une généralisation du processus de Pólya, Acad. Roy. Belgique. Bull. Cl. Sci. 34, 863 (1948).

M 10, 552

Consael, R., Sur quelques processus stochastiques discontinus à deux variables aléatoires, I, Acad. Roy. Belgique. Bull. Cl. Sci. 35, 399 (1949).

M 11, 119

Consael, R., Sur quelques processus stochastiques discontinus à deux variables aléatoires, II, Acad. Roy. Belgique, Bull. Cl. Sci. 35, 743 (1949).

M 11, 256

Coulson, C. A., Note on the random-walk problem Proc. Cambridge Philos. Soc. 43, 583 (1947).

М 9, 96

Cox, D. R., The statistical analysis of congestion, J. Roy. Statist. Soc. Ser. A. 118, 324 (1955).

M 1\%, 277

Daboni, L., Studio delle probabilità subordinate in un caso particolare di processo stocastico, $A n n$. Triestini. Sez. 2, 23 (1951).

M 13, 664

Daniels, H. E., The probability distribution of the extent of a random chain, Proc. Cambridge Philos. Soc. 3\%, 244 (1941).

M 3, 169

Derman, C., Ergodic property of the Brownian motion process. Proc. Nat. Acad. Sci. U.S.A. 40, 1155 (1954).

M 16, 495

-Derman, C., (See K. L. Chung), Pacific J. Math.6, 441 (1956).

Derman, C., A note on nonrecurrent random walks, Proc. Amer. Math. Soc. \%, 762 (1956).

M 18, 681

$\checkmark$ Dmitriev, N., (See A. N. Kolmogoroff), C.R. Acad. Sci. URSS 56, 5 (1947).

Donsker, M. D., Justification and extension of Doob's heuristic approach to the Kolmogorov-Smirnov theorems, Ann. Math. Statist. 23, 277 (1952).

M 13, 853
Doob, J. L., One-parameter families of transformations, Duke Math. J. 4, 752 (1938). $\quad$ Z 20, 47

Doob, J. I., The Brownian movement and stochastic equations, Ann. of Math. 43, 351 (1942). M 4, 17

Doob, J. L., The elementary Gaussian processes, Ann. Math. Statist. 15, 229 (1944). M 6, 89

Doob, J. L., Heuristic approach to the KolmogorovSmirnov theorems, Ann. Math. Statist. 20, 393 (1949).

M 11, 43

Doob, J. L., Continuous parameter martingales, Proc. Symp. Math. Prob. p. 269 (U. Calif. Press, Berkeley 1951).

M 13, 475

Doob, J. L., Semimartingales and subharmonic functions, Trans. Amer. Math. Soc. $\mathbf{9 \% , ~} 86$ (1954).

M 16, 269

Dubrovsky, V., Investigation of purely discontinuous random processes by means of integro-differential equations, Bull. Acad. Sci. URSS. Ser. Math. 8, 107 (1944).

M 6, 160

Dubrovsky, V. M., On purely discontinuous random processes with residual effect, $C$. R. Acad. Sci. URSS 4\%, 79 (1945).

M $\boldsymbol{7}, 129$

$\checkmark$ Dunford, N., Convergence almost everywhere of operator averages, J. Rational Mech. Anal. 5, 129 (1956).

M 18, 987

-Dvoretzky, A., Double points of paths of Brownian

- motion in $n$-space, Acta Sci. Math. Szeged 12, Pars. B, 75 (1950).

M 11, 671

Dvoretzky, A., Some problems on random walk in space, Proc. Sym. Math. Prob., p. 353 (U. Calif. Press, Berkeley 1951).

M 13, 852

-Dvoretzky, A., Multiple points of paths of Brownian motion in the plane, Bull. Res. Council Israel 3, 364 (1954).

M 16, 725

-Dynkin, E. B., Matematičeskie besedy. Zadači o mnogocvetnoŭ raskraske. Zadači iz teorii čisel. Slučănye bluz̆daniya. [Mathematical conversations. Map coloring problems. Problems from number theory. Random walks.], (Gosudarstv. Izdat. Tehn.-Teor. Lit., Moscow 1952).

M 14, 455

Eberl, W., Zur wahrscheinlichkeitstheoretischen Deutung gewisser Mannschaftswettkämpfe, Österreich. Ing.-Arch 10, 148 (1956).

M 18,520

Elfving, G., On compound binomial processes, Försäkringsmatematiska Studier F. Lundberg (Stockholm 1946).

M 8, 391

-Erdos, P., (See A. Dvoretzky), Acta Sci. Math. Szeged 12, Pars B, 75 (1950).

- Erdos, P., (See A. Dvoretzky), Proc. Sym. Math. Prob. p. 353 (U. Cal. Press, Berkeley 1951).

-Erdos, P., (See A. Dvoretzky), Bull. Res. Council Israel 3, 364 (1954).

Everett, C. J., Multiplicative systems, I, Proc. Nat. Acad. Sci. U.S.A. 34, 403 (1948). M 10, 132

- Fagen, R. E., Queuing systems for single and multiple operation, J. Soc. Indust. Appl. Math. 3, 73 (1955).

M 18, 379

Fan, K., Le prolongement des fonctionnelles continues sur un espace semi-ordonné, Revue Sci. 82, 131 (1944).

M 8, 276 
Feller, W., Fluctuation theory of recurrent events, Trans. Amer. Math. Soc. 67, 98 (1949). M 11, 255

Feller, W., The asymptotic distribution of the range of sums of independent random variables, Ann. Math. Statist. 22, 427 (1951).

M 13, 140

Feller, W., Some recent trends in the mathematical theory of diffusion, Proc. Int. Cong. Math. 2, 322 (Amer. Math. Soc., Providence, R.I., 1952).

M 13, 476

Fényes, I., Eine wahrscheinlichkeitstheoretische Begründung und Interpretation der Quantenmechanik, Z. Physik. 132, 81 (1952).

M 15, 78

- Ferrand, J., Sur des suites arithmétiques équiréparties, C. R. Acad. Sci. Paris 224, 516 (1947).

M 8, 391

- Fisz, M., Analytical characterization of a composed, non-homogeneous Poisson process, Studia Math. 15, 328 (1956).

M 18, 605

Florek, K., Remarks on the Poisson stochastic process, I, Studia Math. 13, 122 (1953).

M 14, 1100

- Fortet, R., (See J. Ferrand), C. R. Acad. Sci. Paris 224, 516 (1947).

Fortet, R., Quelques travaux récents sur le mouvement brownien, Ann. Inst. H. Poincaré 11, 175 (1949).

M 11, 731

Fortet, R., Les processus stochastiques en cascades, Trabajos Estadistica 4, 11 (1953).

M 15, 542

- Foster, F. G., On a generalization of Pólya's random-walk theorem, Quart. J. Math., Oxford Ser. 4, 120 (1953).

M 14, 1101

Foster, F. G., On the stochastic matrices associated with certain queuing processes, Ann. Math. Statist. 24, 355 (1953).

M 15, 44

Foster, F. G., A note on Bailey's and Whittle's treatment of a general stochastic epidemic, Biometrika 42, 123 (1955).

M 16, 1130

Fréchet, M., Les éléments aléatoires de nature quelconque dans un espace distancié, Ann. Inst. H. Poincaré 10, 215 (1948).

M 10, 311

Frisch, R., On the inversion of a moving average, Skand. Aktuarie Tidskr. 21, 218 (1938).

$\mathrm{Z} \mathbf{2 0}, 147$

- Fürth, R., Statistical analysis of spontaneous electrical fluctuations, Proc. Phys. Soc. 59, 388 (1947).

M 9, 97

Fürth, R., On the theory of electrical fluctuations, Proc. Roy. Soc. London Ser. A. 192, 593 (1948).

M 9, 452

Gani, J., Some problems in the theory of provisioning and of dams, Biometrika 42, 179 (1955).

M 1\%, 1097

Gani, J., Sufficiency conditions in regular Markov chains and certain random walks. Biometrika 43, 276 (1956).

M 18, 342

Gel'fand, I. M., Generalized random processes, Dokl. Akad. Nauk SSSR 100, 853 (1955).

M 16, 938

Gheorghiu, S., Quelques problèmès concernant la division d'un segment par des points pris au hasard, Acad. R. P. Romine, Stud. Cerc. Mat. 6, 243 (1955).
Gihman, I. I., On a scheme of formation of random processes, Doklady Akad. Nauk, SSSR 58, 961 (1947).

M 9, 293

Girshick, M. A., (See D. Blackwell), Ann. Math. Statist. 1\%, 310 (1946).

Goldstein, S., On diffusion by discontinuous movements, and on the telegraph equation, Quart. J. Mech. Appl. Math. 4, 129 (1951). M 13, 960

Good, I. J., The number of individuals in a cascade process, Proc. Cambridge Philos. Soc. 45, 360 (1949).

M 10, 614

Good, I. J., Random motion on a finite Abelian group, Proc. Cambridge Philos. Soc. 4\%, 756 (1951).

M 13, 363

Good, I. J., Random motion on a finite Abelian group, Proc. Cambridge Philos. Soc. 48, 368 (1952).

M 13, 663

Good, I. J., (See F. G. Foster), Quart. J. Math., Oxford Ser. 4, 120 (1953).

Griffith, L., A theory of the size distribution of particles in a comminuted system, Canadian $J$. Research. Sect. A. 21, 57 (1943). $\quad$ M 4, 249

Halmos, P. R., Invariants of certain stochastic transformations: The mathematical theory of Gambling systems, Duke Math. J. 5, 461 (1939).

Z 22, 247

-Hammersley, J. M., Transposed branching processes, J. Roy. Statist. Soc. Ser. B. 16, 76 (1954).

M 16, 268

Harris, T. E., Branching processes, Ann. Math. Statist. 19, 474 (1948).

$\mathrm{M} \mathbf{1 0}, 311$

-Harris, T. E. (See R. Bellman), Proc. Nat. Acad. Sci. U.S.A. 34, 601 (1948).

Harris, T. E., Some mathematical models for branching processes, Proc. Symp. Math. Prob., p. 305 (Univ. Cal. Press, Berkeley 1951). M 13, 567

-Harris, T. E. (See R. Bellman), Ann. of Math. 55, 280 (1952).

M 13, 664

Hittmair, O., Principe extrémal d'une chaîne de Markoff dans le mouvement brownien, $C . R$. Acad. Sci. Paris 238, 1555 (1954). M 15, 723

-Hodges, J. L., Recurrence-time moments in random walks, Pacific J. Math. 3, 127 (1953).

M 14, 886

Homma, T., On a certain queueing process, Rep. Statist. Appl. Res. Un.Jap. Sci. Engrs. 4, 14 (1955).

M 1\%, 636

Hornby, H., Sur les fonctions aléatoires à symétrie hyperspherique avec composantes gaussiennes et stationnaires du second ordre, C. R. Acad. Sci. Paris 240, 2480 (1955).

M 16, 1128

Hornby, H., Sur une propriété invariante des fonctions aléatoires à symétrie hypersphérique avec composantes gaussiennes et stationnaires, $C$. $R$. Acad. Sci. Paris 241, 353 (1955).

M 16, 1129

Hostinsky, B., Résolution d'un problème général de la theorie de la diffusion, $C$. R. Acad. Sci. Paris 206, 1452 (1938).

Z 18, 414

- Hurwitz, H., Statistical analysis of certain types of random functions, Ann. Math. Statist. 15, 173 (1944). 
Husu, A. P., On some functionals given on processes which are encountered in engineering, Vestnik Leningrad Univ. 11, 89 (1956).

M 1\%, 867

Invrea, R., Il rischio medio di un'operazione assicurativa e l'applicazione di un teorema del Cantelli, Giorn. 1st. Ital. Attuari 12, 167 (1941).

M 8, 390

- Ionescu Tulcea, Sur certaines chaînes à liaisons complètes, C. R. Acad. Sci. Paris 22\%, 667 (1948).

M 10, 311

Itô, K., On a stochastic integral equation, Proc. Japan Acad. 22, 32 (1946).

M 12, 191

Itô, K., Brownian motions in a Lie group, Proc. Japan Acad. 26, 4 (1950).

M 13, 760

Itô, S., On the canonical form of turbulence, Nagoya Math. J. 2, 83 (1951).

M 12, 724

Jackson, R. R. P., Random queueing processes with phase-type service, J. Roy. Statist. Soc. Ser. B. 18, 129 (1956).

M 18, 157

$\checkmark$ Jackson, R. R. P., Some equilibrium results for the queueing process, J. Roy. Statist. Soc. Ser. B. 18, 275 (1956).

M 18, 681

Jánossy, L., Rate of $n$-fold accidental coincidences, Nature 153, 165 (1944).

M 5, 206

Kac, M., On a problem concerning probability and its connection with the theory of diffusion, Bull. Amer. Math. Soc. 46, 534 (1940). M 1, 344

$\checkmark$ Kac, M. (See H. Hurwitz), Ann. Math. Statist. 15, 173 (1944).

Kac, M., Random walk in the presence of absorbing barriers, Ann. Math. Statist. 16, 62 (1945).

М 6, 233

Kakutani, S., On Brownian motions in n-space, Proc. Imp. Acad. Tokyo 20, 648 (1944).

M $\boldsymbol{g}, 315$

Kakutani, S., Two-dimensional Brownian motion and harmonic functions, Proc. Imp. Acad. Tokyo 20, 706 (1944).

M $\boldsymbol{y}, 315$

Kakutani, S., Two-dimensional Brownian motion and the type problem of Riemann surfaces, Proc. Japan Acad. 21, 138 (1945).

M 11, 257

$\checkmark$ Kakutani, S. (See A. Dvoretzky), Acta Sci. Math. Szeged 12, pars B, 75 (1950).

Kakutani, S., Determination of the spectrum of the flow of Brownian motion, Proc. Nat. Acad. Sci. U.S.A. 36, 319 (1950).

M 12, 33

Kakutani, S., Random walk and the type problem of Riemann surfaces, Ann. Math. Studies, 30, p, 95 (Princeton Univ. Press, N.J. 1953). M 15, 25

-Kakutani, S., (See A. Dvoretzky), Bull. Res. Council Israel 3, 364 (1954).

Kallianpur, G., Ergodic property of the Brownian motion process, Proc. Nat. Acad. Sci. U.S.A. 39, 525 (1953).

M 15, 44

Kawata, T., A problem in the theory of queues, Rep. Statist, Appl. Res. Un. Jap. Sci. Engrs. 3, 122 (1955).

M 16, 1035
Kawata, T., On the stochastic process of random noise, Kōdai Math. Sem. Rep. y, 33 (1955).

M 1\%, 636

-Keilson, J., On Brownian motion, Boltzmann's equation, and the Fokker-Planck equation, Quart. Appl. Math. 10, 243 (1952).

M 14, 296

Kemperman, J. H. B., The General One-Dimensional Random Walk with Absorbing Barriers with Appli. cations to Sequential Analysis, (Excelsiors FotoOffset's - Gravenhage, 1950).

M 12, 346

Kemperman, J. H. B., Some remarks on the "random walk," Math. Centrum Amsterdam. Rapport ZW 1951-005, (1951).

M 13, 141

Kendall, D. G., On the generalized "birth-and-death" process, Ann. Math. Statist. 19, 1 (1948).

M 9, 451

Kendall, D. G., On some modes of population growth leading to R. A. Fisher's logarithmic series distribution, Biometrika 35, 6 (1948). M 10, 133

Kendall, D. G., On the role of variable generation time in the development of a stochastic birth process, Biometrika 35, 316 (1948). M 10, 385

Kesten, H., Priority in waiting line problems, Math. Centrum. Amsterdam. Rep. S-211, VP11 (1956).

M 18, 708

Khintchine, A., Theorie der abklingenden Spontaneffiekte, Bull. Acad. Sci. URSS, Sér. Math. Nr. 3, 313 (1938).

Z 19, 224

Khintchine, A. Y., (Mathematičeskie Osnovaniya Statističeskoi Mehaniki. Mathematical Principles of Statistical Mechanics), (OGIZ, Moscow-Leningrad 1943).

M 8, 187

Khintchine, A., The concept of entropy in the theory of probability, Uspehi Matem. Nauk 8, 3 (1953).

M 15, 238

Khintchine, A. I., The notion of entropy in the calculus of probabilities, Acad. Repub. Pop. Romîne An Romîno-Sov. Mat. Fiz. \%, 32 (1954).

M 16, 268

- Kiefer, J., On the theory of queues with many servers, Trans. Amer. Math. Soc. 78, 1 (1955).

M 16, 601

King, G. W., Stochastic methods in quantum mechanics, Proc. Seminar Sci. Computation, p. 42 (I.B.M. Corp., New York 1950).

M 14, 414

King, G. W., Further remarks on stochastic methods in quantum mechanics, Proc. Seminar Sci. Computation, p. 92 (I.B.M. Corp., New York 1951).

M 14, 995

Kitagawa, T., Linear stochastic translatable functional equations and stochastic Cauchy series, Jap. J. Math. 22, 1 (1953).

M 16, 151

Klein, G., A generalization of the classical randomwalk problem, and a simple model of Brownian motion based thereon, Proc. Roy. Soc. Edinburgh. Sect. A. 63, 268 (1952).

M 14, 295

Kolmogoroff, A., Zur Berechnung der mittleren Brownschen Fläche, Physik Z. Sowjetunion 4, 1 (1933).

Z \%, 219

Kolmogoroff, A. N., Branching stochastic processes, C. R. Acad. Sci. URSS 56, 5 (1947). M 9, 46 
$\checkmark$ Kolmogorov, A. N., The calculation of final probabilities for branching random processes, Doklady Akad. Nauk. SSSR 56, 783 (1947).

M 9, 149

-Kolmogorov, A. N., On sums of a random number of random terms, Uspehi Matem. Nauk. 4, No. 4, 168 (1949).

M 11, 119

Kosambi, D. D., Statistics in function space, $J$. Indian Math. Soc. $\boldsymbol{g}, 76$ (1943). M 5, 207

$\checkmark$ Kryloff, N., Sur quelques problèmes de théorie ergodique de systèmes stochastiques, Ann. Chaire Phys. Math. Kiev 4, 243 (1939).

Z 22, 247

Kuznecov, P. I., Quasi-moment functions in the theory of random processes, Doklady Akad. Nauk 94, 615 (1954).

M 15, 883

Lampard, D. G., Generalization of the WienerKhintchine theorem to nonstationary processes, J. Appl. Phys. 25, 802 (1954).

M 15, 969

Lauwerier, H. A., A linear random walk with a partly reflecting partly absorbing barrier, Appl. Sci. Research B. 2,294 (1952).

M 13, 852

-Ledermann, W., Spectral theory for the differential equations of simple birth and death processes, Philos Trans. Roy. Soc. London A. 246, 321 (1954).

M 15, 625

Lehman, R. S., A problem on random walk (1933), Proc. Symp. Math. Prob. p. 263 (Univ. Calif. Press, Berkeley, 1951).

M 13, 363

Leonov, Y. P., On filtering nonstationary random functions, Artomat. $i$ Telemeh. 1\%, 97 (1956).

M 1\%, 1100

-Leontowitsch, M. (See A. Kolmogoroff), Physik. Z. Sowjetunion 4, 1 (1933).

Levinson, N., The Wiener RMS (root mean square) error criterion in filter design and prediction, $J$. Math. Phys. Mass. Inst. Tech. 25, 261 (1947).

M 8, 391

Levinson, N., A heuristic exposition of Wiener's mathematical theory of prediction and filtering, J. Math. Phys. Mass. Inst. Tech. 26, 110 (1947).

M 9, 46

Lévy, P., Mouvement brownien linéaire et mouvement brownien plan, C. R. Acad. Sci. Paris $\mathbf{2 0 9 ,}$ 140 (1939).

M 1, 22

Lévy, P., Le mouvement brownien plan, Amer. J. Math. 62, 487 (1940).

M 2,107

Lévy, P., Un théorème d'invariance projective relatif au mouvement brownien, Comment. Math. Helv. 16, 242 (1944).

M 6, 5

Lévy, P., Une propriété d'invariance projective dans le mouvement brownien, C. R. Acad. Sci. Paris 219, 378 (1944).

M $\%, 314$

Lévy, P., Sur le mouvement brownien dépendant de plusieurs paramètres, $C$. R. Acad. Sci. Paris 220, 420 (1945).

M 7,130

Lévy, P., Processus Stochastiques et Mouvement Brownien, Suivi d'une note de M. Loève, Gauthier Villars, Paris, 1948).

M 10, 551

Lévy, P., Fonctions aléatoires laplaciennes, $C . R$. Acad. Sci. Paris 229, 1057 (1949).

M 11, 376
Lévy, P., Sur l'aire comprise entre un arc de la courbe du mouvement brownien plan et sa corde, $C . R$. Acad. Sci. Paris 230, 432 (1950).

M 12, 191

Lévy, P., La mesure de Hausdorff de la courbe du mouvement brownien à $\mathrm{n}$ dimensions, $C$. R. Acad. Sci. Paris 233, 600 (1951).

M 13, 363

Lévy, P., La measure de Hausdorff de la courbe du mouvement brownien, Giorn. 1st. Ital. Attuari 16, 1 (1954).

M 16, 268

Lévy, P., Le mouvement brownien, Mémor. Sci. Math., No. 126, (Gauthier-Villars, Paris, 1954).

M 16, 601

Lévy, P., Le mouvement brownien à $n=2 p+1$ paramètres, II, C. R. Acad. Sci. Paris $\mathbf{2 3 9 ,} 1584$ (1954).

M 16, 495

Lévy, P., Le mouvement brownien à $n=2 p+1$ paramètres, C. R. Acad. Sci. Paris \$39, 1181 (1954).

M 16, 495

Lévy, P., Sur une classe de fonctions aléatoires gaussiennes, C. R. Acad. Sci. Paris 240, 1308 (1955).

M 16, 939

Lévy, P., Le mouvement brownien à $n=2 p+1$ paramètres, III, C. R. Acad. Sci. Paris 240, 1043 (1955).

M 16, 939

Lévy, P., Fonctions aléatoires a corrélation linéaire, C. R. Acad. Sci. Paris 242, 2095 (1956).

Lévy, P. Random functions: a Laplacian random function depending on a point of Hilbert space, Univ. California Publ. Statist. 2, 195 (1956).

M 18, 770

Lévy, P., Intégration d'une équation intégrale non linéaire, C. R. Acad. Sci. Paris 242, 1252 (1956).

M 1\%, 978

Lévy, P., Fonctions aléatoires à corrélation linéaire, C. R. Acad. Sci. Paris 242, 1575 (1956).

M 18,978

Lin, C. C., On the motion of a pendulum in a turbulent fluid, Quart. Appl. Math. 1, 43 (1943).

M 4, 227

Loève, M., Sur les systèmes d'évènements; application à deux théorèmes classiques, $C . R$. Acad. Sci. Paris 212, 261 (1941).

M 3, 3

-Longuet-Higgins, M. S. (See Cartwright, D. E.), Proc. Roy. Soc. London A. 23\%, 212 (1956).

Lopuszański, J., Some remarks on the asymptotic behaviour of the cosmic ray cascade for large depth of the absorber. II. Asymptotic behaviour of the probability distribution function, Nuovo Cimento, 2, Supplemento, 1150 (1955). M 1\%, 867

Luchak, G., The solution of the single-channel queuing equations characterized by a time-dependent Poisson-distributed arrival rate and a general class of holding times, Operations Res. 4, 711 (1956).

M 18, 707

Lüneburg, R., Das problem der Irrfahrt ohne Richtungsbeschränkung und die Randwertaufgabe der Potentialtheorie, Math. Annalen 104, 700 (1931).

Z 1, 399

MacDonald, D. K. C. (See R. Fürth), Proc. Phys. Soc. 59, 388 (1947). 
Marcinkiewicz, J., Sur une propriété du mouvement brownien, Acta Litt. Sci. Szeged 9, 77 (1939).

-Marczewski, E., (See K. Florek), Studia Math. 13, 122 (1953).

Marczewski, E., Remarks on the Poisson stochastic process, II, Studia Math. 13, 130 (1953).

M 14, 1100

Marinescu, G., (See Ionescu), C. R. Acad. Sci. Paris 22\%, 667 (1948).

Maruyama, G., Fourier analytic treatment of some problems on the sums of random variables, Nat. Sci.Rep. Ochanomizu Univ. 6, 7 (1955).

M 18, 341

Maruyama, G., On the Poisson distribution derived from independent random walks, Nat. Sci. Rep. Ochanomizu Univ. 6, 1 (1955).

M 18, 341

- Mathews, P. M., (See A. Ramakrishnan), Proc. Indian Acad. Sci. Sect. A. 43, 84 (1956).

Matschinski, M., Quelques remarques sur les processus stochastiques. Le processus stochastique dans une population, C. R. Acad. Sci. Paris 235, 1362 (1952).

M 14, 485

Matschinski, M., Equations générales des processus stochastiques. Population "holostochastique" et population "semistochastique", C. R. Acad. Sci. Paris 236, 580 (1953).

M 14, 772

- McCrea, W. H., Random paths in two and three dimensions, Proc. Roy. Soc. Edinburgh 60, 281 (1940).

M ?, 107

McKean, H. P., Hausdorff-Besicovitch dimension of Brownian motion paths, Duke Math. J. 22, 229 (1955)

M 16, 1036

McKean, H. P., Sample functions of stable processes, Ann. of Math.61, 564 (1955). M 16, 1036

Meinesz, M., The problem of the gambler's ruin, Statistica, Neerlandica. 10, 87 (1956). M 18, 342

Milatz, J. M. W., Brownian motion, Nederl. Tijdschr. Natuurkunde 8, 19 (1941).

M $\%, 315$

Montroll, E. W., Markoff chains and excluded volume effect in polymer chains, J. Chem. Phys. 18, 734 (1950).

M 12, 114

Moran, P. A. P., Some theorems on time series, I, Biometrika 34, 281 (1947).

M 9, 361

Moran, P. A. P., The oscillatory behaviour of moving averages, Proc. Cambridge Philos. Soc. 46, 272 $(1950)$.

M 11, 672

Moran, P. A. P., A probability theory of dams and storage systems: modifications of the release rules, Austral. J. Appl.Sci. 6, 117 (1955). M 1\%, 1097

Morgenstern, D., Úber die Differentialgleichung des reinen Geburtsprozesses in der Wahrscheinlichkeitsrechnung, Math. Nachr. 13, 57 (1955).

M 16, 1129

Morse, P. M., Stochastic properties of waiting lines, J. Operations Res. Soc. Amer. 3, 255 (1955).

M 1\%, 51

-Morton, K. W., (See J. M. Hammersley), J. Roy. Statist. Soc. Ser. B. 16, 76 (1954).

Motzkin, T. S., Neighbor sets for random walks and difference equations, Symp. Monte Carlo Methods, Univ. Florida (John Wiley 1956).

M 18, 152

Mycielski, J., On the distances between signals in the non-homogeneous Poisson stochastic process, Studia Math. 15, 300 (1956).

M 18, 520

Nakagami, M., On the resultant intensity of a number of vibrations whose phase are at random, Nippon Elec. Commun. Engrg., No. 19, 129 (1940).

M 2,229

Nakagami, M., Study on the resultant amplitude of many vibrations whose phases and amplitudes are at random, Nippon Elec. Commun. Engrg., No. 22, $69(1940)$

M 2,229

Natta, G., Leggi di ripartizione delle singole specie molecolari nei prodotti di una catena di reazioni successive, 1st. Lombardo Sci. Lett. Cl. Sci. Mat. Nat. Rend. 9, 307 (1945).

M 8, 281

Nickols, D. G., (See R. R. P. Jackson), J. Roy. Statist. Soc. Ser. B. 18, 275 (1956).

- Nisida, T., (See T. Sirao), Nagoya Math. J. 4, 97 (1952)

Nisida, T., Note on Brownian motions with a parameter space $\mathrm{R}^{\mathrm{n}}$, Math. Japon. 3, 85 (1954).

M 16, 1130

-Ohno, O., (See M. Nakagami), Nippon Elec. Commun. Engrg., No. 19, 129 (1940).

Onicescu, O., Chaînes continues à liaisons complètes, Rev. Univ. "C. I. Parhon" Politehn. Bucurest 3, 73 (1954).

M 1\%, 980

Osida, I., Brownian motion in a plasto-elastic medium, Proc. Phys.-Math. Soc. Japan 24, 599 (1942).

M $\%, 315$

Ostene, E., Sur le principe ergodique dans les chaînes de Markoff à éléments variables, $C . R$. Acad. Sci. Paris 199, 175 (1934).

Z 9, 264

Otter, R., The multiplicative process, Ann. Math. Statist. 20, 206 (1949).

M 11, 41

Patlak, C. S., Random walk with persistence and external bias, Bull. Math. Biophys. 15, 311 (1953).

M 18, 424

Picard, H. C., Two random movements in a plane, Simon Stevin 30, 25 (1954).

M 15, 884

Pignedoli, A., Su movimenti di tipo Browniano, $J$. Rational Mech. Anal. 4, 579 (1955). M 16, 1130

Pinsker, M. S., The quantity of information about a Gaussian random stationary process, contained in a second process connected with it in a stationary manner, Dokl. Akad. Nauk. SSSR 99, 213 (1954).

M 16, 495

Potoček, J., La diffusion et la notion de réversibilité de M. Kolmogoroff, Bull. Int. Acad. Sci. Bohême 1939, 1, (1939).

M 1, 247

- Prohorov, Y. V., (See A. N. Kolmogorov) Uspehi Matem. Nauk 4, 168 (1949).

Ramakrishnan, A., Stochastic processes associated with random divisions of a line, Proc. Cambridge Philos. Soc. 49, 473 (1953).

M 14, 1100

- Ramakrishnan, A., Stochastic processes associated with a symmetric oscillatory Poisson process, Proc. Indian Acad. Sci. Sect. A. 43, 84 (1956).

M 1\%, 980

-Reuter, G. E. H., (See W. Lederman) Philos. Trans. Roy. Soc. London A. 246, 321 (1954). 
Rider, P. R., Truncated binomial and negative binomial distributions, J. Am. Statist. Assoc. 50, 877 (1955).

M 1\%, 169

- Riordan, J., (See R. E. Fagen) J. Soc. Indust. Appl. Math. 3, 73 (1955).

$\checkmark$ Robbins, H., (See G. Kallianpur) Proc. Nat Acad. Sci. U.S.A. 39, 525 (1953).

Romanovskil, V. I., On the probabilities of the recurrence of cycles in polycyclic chains, Acta Univ. Asiae Mediae, Fasc. 7, 20 pp. (1946).

M 8, 522

Rosenblatt, A., Sur le concept de contagion de M. G. Pólya dans le calcul del probabilités. Divers schemes. Application à la peste bubonique au Pérou, Actas Acad. Ci. Lima 3, 186 (1940).

M 3, 2

$\checkmark$ Rosenblatt, M., (See J. L. Hodges) Pacific J. Math. 3, 127 (1953).

$\checkmark$ Runnenburg, J. T. L., (See H. Kesten) Math. Centrum Amsterdam. Rep. S 211, VP11 (1956).

Ryll-Nardzewski, C., (See K. Florek) Studia Math. 13, 122 (1953).

Ryll-Nardzewski, C., On the non-homogeneous Poisson process, I, Studia Math. 14, 124 (1954).

M 15, 883

Ryll-Nardzewski, C., Remarks on the Poisson stochastic process. III. On a property of the homogeneous Poisson process, Studia Math. 14, 314 (1955).

M 16, 723

Sarymsakov, T. A., Sur les chaînes de Bruns, $C . R$. (Doklady) Acad. Sci. URSS 49, 241 (1945).

M 8, 38

Satterthwaite, F. E., Generalized Poisson distribution, Ann. Math. Statist. 13, 410 (1942).

M 4, 163

von Schelling, H., Das Alles-oder-Nichts-Gesetz gedeutet als Endergebnis einer Auslösungsfolge, Abh. Preuss. Akad. Wiss. Math.-Nat. Kl. 1944, 25 pages (1944).

- Schwartz, J. T., (See N. Dunford), J. Rational Mech. Anal. 5, 129 (1956).

M 8, 214

ast vanov, B. A., On the theory of branching random processes, Doklady Akad. Nauk SSSR 59, 1407 (1948).

M 9, 451

-Sevast'yanov, B. A., (See A. N. Kolmogorov) Doklady Akad. Nauk SSSR 56, 783 (1947).

Shenton, L. R., A semi-infinite random walk with discrete steps, Proc. Cambridge Philos. Soc. 51, 442 (1955).

M 16, 1035

- Sirao,T., On some asymptotic properties concerning Brownian motion, Nagoya Math. J. 4, 97 (1952).

M 13, 761

Sirao, T., On some asymptotic properties concerning homogeneous differential processes, Nagoya Math. J. 6, 95 (1953).

M 15, 449

Sirao, T., On the uniform continuity of Wiener process, J. Math. Soc. Japan 6, 332 (1954).

M 16, 725

Siraždinov, S. H., The ergodic principle for nonstationary Markov chains, Doklady Akad. Nauk SSSR \%1, 829 (1950).

M 11, 606
Siraždinov, S. H., Refinement of limiting theorems for stationary Markov chains, Doklady Akad. Nauk SSSR 84, 1143 (1952).

M 14, 187

Siraždinov, S. H., Limit theorems for stationary Markov chains in continuous time, Dokl. Akad. Nauk SSSR 98, 905 (1954).

M 16, 494

Smith, W. L., On renewal theory, counter problems and quasi-Poisson processes, Proc. Cambridge Philos. Soc. 53, 175 (1957).

M 18, 832

Snell, J. L., Applications of martingale system theorems, (Thesis, University of Illinois, 1951).

M 13, 568

Snell, J. L., Applications of martingale system theorems, Trans. Amer. Math. Soc. 73, 293 (1952).

M 14, 295

Spitzer, F., On a class of random variables, Proc. Amer. Math. Soc. 6, 494 (1955). $\quad$ M 16, 938

Steinhaus, H., Sur les fonctions indépendantes. VII. (Un essaim de points à l'intérieur d'un cube), Studia Math. 10, 1 (1948).

M 9, 292

Steinhaus, H., Sur les fonctions indépendantes. X. Équipartition de molécules dans un récipient cubique, Studia Math. 13, 1 (1953). M 16, 378

-Storer, J. E., (See J. Keilson) Quart. Appl. Math. 10, 243 (1952).

- Stratonovič, R. L., (See P. I. Kuznecov) Doklady Akad. Nauk SSSR 94, 615 (1954).

Takács, L., On stochastic processes connected with certain physical registration mechanisms, Magyar Tud Akad. Mat. Fiz. Oszt. Közl. 4, 571 (1954).

M 16, 723

Takács, L., Investigation of waiting time problems by reduction to Markov processes, Acta Math. Acad. Sci. Hungar 6, 101 (1955). $\quad$ M 17,51

Takács, L., On processes of happenings generated by means of a Poisson process, Acta Math. Acad. Sci. Hungar 6, 81 (1955).

M 1\%, 51

Takács, L., On stochastic processes connected with certain physical recording apparatuses, Acta Math. Acad. Sci. Hungar 6, 363 (1955).

M 17, 867

Takács, L., On a probability treatment of electrontube plate-current oscillation, Magyar Tud, Akad. Mat. Fiz. Tud. Oszt. Közl. 6, 27 (1956).

M 1\%, 1218

Takács, L., On secondary stochastic processes generated by recurrent processes, Acta Math. Acad. Sci. Hungar 8, 17 (1956).

M 1\%, 1217

Takano, K., Note on Wiener's prediction theory, Ann. Inst. Statist. Math. Tokyo 5, 67 (1954).

M 16, 151

Talacko, J., Perk's distributions and their role in the theory of Wiener's stochastic variables, Trabajos Estadist $\boldsymbol{y , ~} 159$ (1956).

M 18, 770

Taylor, S. J., The Hausdorff $\alpha$-dimensional measure of Brownian paths in $\mathbf{n}$-space Proc. Cambridge Philos. Soc. 49, 31 (1953).

M 14, 663

-Terletsky, J., (See V. Vladimirsky) Akad. Nauk SSSR Zhurnal Eksper. Teoret. Fiz. 15, 258 (1945).

-Tihonov, V. I., (See P. I. Kuznecov) Doklady Akad. Nauk SSSR 94, 615 (1954). 
Uhlenbeck, G. E., (See M. C. Wang) Rev. Modern Phys. 1\%, 323 (1945).

$\checkmark$ Ulam, S., (See C. J. Everett) Proc. Nat. Acad. Sci. U. S. A. 34, 403 (1948).

Urbanik, K., Some remarks on the asymptotic behaviour of the cosmic ray cascade for large depth of the absorber, Nuovo Cimento. 2 Supplemento, 1147 (1955).

M 1\%, 867

Urbanik, K., On a stochastic model of a cascade, Bull. Acad. Polon. Sci. Cl. III 3, 349 (1955).

M 1\%, 276

Urbanik, K., On a problem concerning birth and death processes, Acta Math. Acad. Sci. Hungar y, 99 (1956).

M 18, 77

-Urbanik, K., (See M. Fisz) Studia Math. 15, 328 (1956).

Uspenskii, V. A., (See E. B. Dynkin) (Gosudarstv. Izdat. Tehn.-Teor. Lit., Moscow, 1956).

Vajda, S., Introduction to a mathematical theory of the graded stationary population, Mitt. Verein. Schweiz. Versich.-Math.48,251 (1948). M 10,465

- Van Kampen, E. R., A limit theorem for probability distributions on lattices, Amer. J. Math. 61, 965 (1939).

M 1, 63

Ville, J. A., Sur une problème de géométrie suggéré par l'étude du mouvement brownien, $C$. R. Acad. Sci. Paris 215, 51 (1942).

M 5, 125

-Vladimirsky, V., Hydrodynamical theory of translational Brownian motion, Akad. Nauk SSSR. Zhurnal Eksper. Teoret. Fiz. 15, 258 (1945).

M $\%, 314$

Volberg, O., Problème de la queue stationnaire et nonstationnaire, C.R. (Doklady) Acad. Sci. URSS 24, 657 (1939).

M 1, 345

Vorovič, I. I., On stability of motion for random disturbances, Izv. Akad. Nauk SSSR. Serv. Mat. 20, 17 (1956).

M 1\%, 977

$\checkmark$ Wang, M. C., On the theory of the Brownian motion, II, Rev. Modern Phys. 1\%, 323 (1945). M \%, 130 Wasow, W., On the duration of random walks, Ann. Math Statist. 22, 199 (1951).

M 14, 994

Wasow, W., On the mean duration of random walks, J. Research Nat. Bur. Standards 46, 462 (1951).

M 13, 960

Wassmuth, N., Beiträge zur Theorie der Brownschen Bewegung, C. R. Acad. Sci. URSS 1\%, 307 (1937).

Z 18, 321

Waugh, W.A. O'N., On age-dependent birth and death process, Biometrika 42,291 (1955). M 1\%, 276

$\checkmark$ Whipple, F. J. W., (See W. H. McCrea) Proc. Roy. Soc. Edinburgh 60, 281 (1940).

Whittle, P., The outcome of a stochastic epidemic a note on Bailey's paper, Biometrika 42,116 (1955).

M 16, 1130

-Wiener, N., The discrete chaos, Amer. J. Math. 65, 279 (1943).

M 4, 220

Wigner, E. P., Characteristic vectors of bordered matrices with infinite dimensions, Ann. of Math. 62, 548 (1955).

M 1\%, 1097
-Wintner, A., (See E. R. van Kampen) Amer. J. Math. 61, 965 (1939).

-Wintner, A., (See N. Wiener) Amer. J. Math. 65, 279 (1943).

Wishart, D. M. G., A queueing system with $\chi^{2}$ service-time distribution, Ann. Math. Statist. 2\%, 768 (1956).

M 18, 157

$\checkmark$ Wolfowitz, J., (See J. Kiefer) Trans. Amer. Math. Soc. 78, 1 (1955).

Woodward, P. M., A statistical theory of cascade multiplication, Proc. Cambridge Philos. Soc. 44, 404 (1948).

M 10, 133

Yaglom, A. M., Certain limit theorems of the theory of branching random processes, Doklady Akad. Nauk SSSR 56, 795 (1947).

M 9, 149

Yaglom, A. M., On the statistical reversibility of Brownian motion, Mat. Sbcrnik 24, 457 (1949).

M 11, 41

Yosida, K., Stochastic processes built from flows, Proc. Japan Acad. 26, No. 8, 1 (1950). M 13, 760

Yosida, K., Abstract integral equations and the homogeneous stochastic process, Proc. Imp. Acad. Jap. 14, 286 (1938).

Z 19, 414

Yosida, K., An extension of Fokker-Planck's equation, Proc. Japan Acad. 25, No. 9, 1 (1949).

M 12, 270

Yosida, K., Integration of Fokker-Planck's equation with a boundary condition, J. Math. Soc. Japan 3, 69 (1951).

M 13, 560

Yosida, K., Integrability of the backward diffusion equation in a compact Riemannian space, Nagoya Math. J. 3, 1 (1951)

M 13, 560

Yosida, K., On Brownian motion in a homogeneous Riemannian space, Pacific J. Math. 2, 263 (1952).

M 14, 387

Zolotarëv, V. M., On a problem from the theory of branching random processes, Uspehi Matem. Nauk 9, 147 (1954).

M 15, 806

Zwinggi, E., Berechnung und Darstellung der abhängigen und unabhängigen Wahrscheinlichkeiten, Mitt. Verein. Schweiz. Versich. Math. 49, 179 (1949).

M 11, 257

\section{E. Applications}

Adler, H. A., A new approach to probability problems in electrical engineering, Trans. Amer. Inst. Elec. Engrs. 65, 630 (1946). $\quad$ M 8, 214

Ammeter, H., A generalization of the collective theory of risk in regard to fluctuating basicprobabilities, Skand. Aktuarietidskr. 31, 171 (1948).

M 10, 463

Arley, N., On the Theory of Stochastic Processes and Their Application to the Theory of Cosmic Radiation, (John Wiley \& Sons, Inc., N.Y., 1948).

M 10, 464

Bailey, N. T. J., The total size of a general stochastic epidemic, Biometrika 40, 177 (1953). M 14, 1101 
Bartlett, M. S., On the use of the characteristic functional in the analysis of some stochastic processes occurring in physics and biology, Proc. Cambridge Philos. Soc. 47, 65 (1951). M 12, 620 -Bass, J., Sur la connexion aléatoire d'un fluide. Application a la turbulence, C. R. Acad. Sci. Paris 220, 165 (1945).

$\mathrm{M} \boldsymbol{\gamma}, 130$

Bass, J., Les méthodes modernes du calcul des probabilitiés et leur application au problème de la turbulence, (Group. Français Develop. Rech. Aeronaut. Rap. Tech. No. 28, 1946).

M 11, 699

Bass, J., Sur le corpuscule aléatoire à masse aléatoire, C. R. Acad. Sci. Paris 225, 38 (1947). M 9, 47

Bass, J., Les bases d'une théorie statistique de la turbulence, Proc. Seventh Internat. Congress Appl. Mech., 2, 212 (1948).

M 11, 624

Bass, J., Lois de probabilité, équations hydrodynamiques et mécanique quantique, Revue Sci. 86, 643 (1948).

M 12, 229

Bellman, R., A survey of the mathematical theory of time-lag, retarded control, and hereditary processes (The Rand Corp., Santa Monica, Calif., 1954).

M 15, 962

Bennett, W. R., Spectra of quantized signals, Bell System Tech. J. 2\%, 446 (1948).

M 10, 133

- Bentzel, R., On statistical demand analysis from the viewpoint of simultaneous equations, Skand. Aktuarietidskr. 29, 95 (1946).

M 8, 216

Bhabha, H. J., On the stochastic theory of continuous parametric systems and its application to electron cascades, Proc. Roy. Soc. London, Ser. A. 202, 301 (1950).

M 12, 191

Blanc-Lapierre, A., Sur certaines fonctions aléatoires stationnaires. Application á l'étude des fluctuations á la structure électronique de l'électricite (Publ. Labor. École Norm. Sup., Sér. Math., No. 1, 1945).

M 8, 38

Blanc-Lapierre, A., Effet Schottky. Fluctuations dans les amplificateurs linéaires et dans les détecteurs, Bull. Soc. Française Electriciens 5, 9 (1945).

M 8, 38

Blanc-Lapierre, A., Sur l'effet de scintillation, C. R. Acad. Sci. Paris 221, 375 (1945). M \%, 211

-Blanc-Lapierre, A., Fluctuations dans les grandeurs physiques quasi sinusoidales, J. Phys. Radium $\%, 153$ (1946).

M 8, 216

Blanc-Lapierre, A., Etude des fluctuations produites par l'effet de grenaille dans les amplificateurs, Revue Sci. 84, 75 (1946).

M 8, 216

-Blanc-Lapierre, A., Fluctuations dans les grandeurs physiques quasi sinusoidales, $C$. R. Acad. Sci. Paris 22R, 1324 (1946). M g, 460

-Blanc-Lapierre, A., Théorie des fonctions aléatoires. Applications à divers phénomènes de fluctuation. Avec un chapitre sur la mécanique des fluides par J. Kampé de Fériet (Masson et Cie, Paris, 1953).

M 15, 883

Bothe, W., Zur Theorie der Bremsung von Neutronen, Z. Physik 125, 210 (1948). M 11, 298

Bouchman, E. N., Problems of congestion in telephony, J. Appl. Math. Mech. 6, 247 (1942).

M 4, 103
Brockmever, E., The life and works of $A . K$. Erlang (Trans. Danish Acad. Tech. Sci., 1948).

M 10, 385

Buchman, E. N., The problem of waiting time, Akad. Nauk SSSR. Prikl. Mat. Meh. 11, 475 (1947).

M 9, 194

$\checkmark$ Bunimovich, V. I., On the distribution of the number of large deviations in electric fluctuations, C. R. Acad. Sci. URSS 53, 21 (1946). M 8, 392

- Bush, R. R., A stochastic model with applications to learning, Ann. Math. Statist. 24, 559 (1953).

M 15, 449

Cantelli, F. P., Osservazioni sulla formula di Hattendorff, Giorn. 1st. Ital. Attuari 11, 261 (1940).

M 8, 390

Cantelli, F. P., I fondamenti matematici della tecnica delle assicurazioni, Giorn. 1st. Ital. Attuari 13, 1 (1942).

M 8, 215

Casal, P., Statistique d'un champ homogène de vecteurs aléatoires de divergence nulle. Application à la turbulence homogène, $C$. R. Acad. Sci. Paris 226, 870 (1948).

M 9, 452

Casal, P., Étude des champs de vecteurs aléatoires, appliquée à la cinématique des fluides turbulents, Bull. Soc. Math. France $\mathbf{g}, 141$ (1949). M 11, 624

Chandrasekhar, S., Stochastic problems in physics and astronomy, Rev. Modern. Phys. 15, 1 (1943).

M 4, 248

Chandrasekhar, S., Brownian motion, dynamical friction and stellar dynamics, Dialectica 3, 114 (1949).

M 11, 118

Coz Campos, D., On the generalization of the law of radial concentration in the case of a spherical cell, Revista Ci., Lima 4\%, 275 (1945). M $\quad$ \%, 319 Cramér, H., Deux conférences sur la théorie des probabilités, Skand. Aktuarietidskr. 1941, 34 (1941).

M 3, 169

Cunningham, L. B. C., Random processes in problems of air warfare, Suppl. J. Roy. Statist. Soc. 8, 62 (1946).

M 8, 282

Davies, R. W., The connection between the Smoluchowski equation and the Kramers-Chandrasekhar equation, Physical Rev. 93, 1169 (1954). M 15, 723

- Dedebant, G., La mécanique des fluides turbulents fondée sur des concepts statistiques, Thalès $\mathbf{4 ,} 151$ (1940).

М 9, 47

- Dedebant, G., Mécanique aléatoire. I. Le calcul aléatoire (suite), II. Applications physiques. Portugaliae Phys. 1, 179 (1945).

M $\boldsymbol{\gamma}, 129$

Dedebant, G., (See J. Bass), C. R. Acad. Sci. Paris 220, 165 (1945).

Doob, J. L., Renewal theory from the point of view of the theory of probability, Trans. Amer. Math. Soc. 63, 422 (1948).

M 9, 598

Doob, J. L., Time series and harmonic analysis, Proc. Symp. Math. Prob., p. 303 (U. Cal. Press, Berkeley 1949).

M 10, 385

Dubourdieu, J., Sur une généralisation d'un théorème de M.B. de Finetti et son application à la théorie collective du risque, C. R. Acad. Sci. Paris 224, 514 (1947).

M 8, 390 
Egerváry, E., On a certain point of the kinetic theory of gases, Studia Math. 12, 170 (1951).

M 13, 761

Epstein, B., The mathematical description of certain breakage mechanisms leading to the logarithmiconormal distribution, J. Franklin Inst. 244, 471 (1947).

M 9, 360

Epstein, B., Statistical aspects of fracture problems, J. Appl. Phys. 19, 140 (1948).

M 9, 360

$\checkmark$ Ernst, M. L., (See P. M. Morse), J. Operations Res. Soc. Amer. 2, 444 (1954).

Feather, N., On the statistics of random distributions of paired events, with applications to the results obtained in the use of the interval selector with practicle counters, Proc. Cambridge Philos. Soc. 39, 84 (1943).

M 4, 223

Feller, W., Die Grundlagen der Volterraschen Theorie des Kampfes ums Dasein in wahrscheinlichkeitstheoretischer Behandlung, Acta Bioth. Ser. A. 5, 11 (1939).

M 1, 22

Feller, W., On the theory of stochastic processes, with particular reference to applications, Proc. Symp. Math. Prob. p. 403 (Univ. Calif. Press, Berkeley 1949).

M 10, 385

Feller, W., Diffusion processes in genetics, Proc. Symp. Math. Prob. p. 227, (Univ. Calif. Press, Berkeley 1951).

M 13, 671

de Finetti, B., Il problema dei "Pieni," Giorn. 1st. Ital. Attuari. 11, 1 (1940).

M 8, 390

Flanagan, J. E., Coding to achieve Markov type redundancy, J. Math. Phys. 33, 258 (1954).

M 16, 601

Fomin, S., On the theory of dynamical systems with continuous spectrum, Doklady Akad. Nauk SSSR. 6\%, 435 (1949).

M 11, 117

Fortet, R., Sur la probabilité de perte d'un appel téléphonique dans un groupe de $x$ sélecteurs commandés par un orienteur unique, $C . R$. Acad. Sci. Paris 226, 159 (1948).

M 9, 361

Fortet, R., Sur la probabilité de perte d'un appel téléphonique, C. R. Acad. Sci. Paris 226, 1502 (1948).

M 9, 518

- Fortet, R., (See Blanc-Lapierre), (Masson et cie, Paris 1953).

Franckx, E., L'évolution des collectivités, Mitt. Verein. Schweiz. Versich..-Math. 45, 279 (1945).

M 10, 310

Franckx, E., L'évolution des collectivités, Assoc. Actuair. Belges. Bull. 51, 31 (1946). M \%, 311

Frenkiel, F. N., On third-order correlation and vorticity in isotropic turbulence, Quart. Appl. Math. 6, 86 (1948).

M 9, 520

Frenkiel, F. N., On the kinematics of turbulence, J. Aeronaut. Sci. 15, 57 (1948). M 9, 452

-Garber, H. N., (See P. M. Morse), J. Operations Res. Soc. Am. 2, 444 (1954).

García, G., On the integration of the equation of diffusion in the case of a spherical cell, Revista $\mathrm{Ci}$., Lima 46, 417 (1944).

M 6, 240
García, G., On the integration of the equation of diffusion in the case of a spherical cell, Actas Acad. Ci., Lima 8, 61 (1945).

M \%, 133

Gebelein, H., Turbulenz Physikalische Statistik und Hydrodynamik. (Julius Springer, Berlin 1935).

Z 12, 268

Giltay, J., Static and transient statistics in telephonetraffic problems, Appl. Sci. Research B. 1, 413 (1950).

M 12, 270

Granovsky, V. L., The deionization of gas in the after-discharge period, Bull. Acad. Sci. URSS, Sér. Physique 4, 419 (1938).

$\mathrm{Z} \mathbf{2 0}, 417$

Grenander, U., Stochastic processes and statistical inference, Ark. Mat. 1, 195 (1950). M 12, 511

-Grenander, U., An extension of a theorem of G. Szegö and its application to the study of stochastic processes, Trans. Amer. Math. Soc. \%6, 112 (1954).

M 15, 448

Grosjean, C. C., Solution of the non-isotropic random flight problem in the k-dimensional space, Physica 19, 29 (1953).

M 14, 772

-Grün, F., Zur Behandlung der Brownschen Bewegung mit Hilfe der Langevin-Gleichung, Helvetica Phys. Acta 2\%, 417 (1954). M 16, 268

-Halstrom, H. L., (See E. Brockmeyer), (Trans. Danish Acad. Tech. Sci. 1948).

Hearon, J. Z., Nonlinear diffusion in metabolic systems, Bull. Math. Biophys. 15, 15 (1953).

M 14, 781

Hearon, J. Z., Comments on the approximate solution of the diffusion equation. II., Bull. Math. Biophys. 15, 111 (1953).

M 14, 1000

- Heinrich, G., Zur Behandlung der Diffusionsvorgänge in der Atmosphäre, Acta Physica Austriaca 4, 160 (1950). M 12, 788

- Hermans, J. J., The statistics of stiff chains, with applications to light scattering, Physica 18, 951 (1952).

M 14, 664

Hilmy, H., Sur la recurrence ergodique dans les systèmes dynamiques, Rec. Math. Moscou, 7,101 (1940).

$\mathrm{Z} \mathbf{2 3}, 146$

Hole, N., On the statistical treatment of counting experiments in nuclear physics, Ark. Mat. Astr. Fys. 33A, No. 11 (1946).

M 8, 282

Hollingsworth, C. A., Solutions of some problems concerning long random coils, J. Chem. Phys. 20, 1580 (1952).

M 14, 486

Horner, F., A problem on the summation of simple harmonic functions of the same amplitude and frequency but of random phase, Philos. Mag. 3\%, 145 (1946).

M 9, 97

Hynd, W. R. B., (See L. B. C. Cunningham), Suppl. J. Roy. Statist. Soc. 8, 62 (1946).

Jánossy, L., Study of a stochastic process arising in the theory of the electron multiplier, Acta Math. Acad. Sci. Hungar. 2, 165 (1951).

M 14, 388

Jánossy, L., Studies on the theory of cascades, Acta Phys. Acad. Sci. Hungar. 2, 289 (1952).

M 15, 330

-Jensen, A., (See E. Brockmeyer), (Trans. Danish Acad. Tech. Sci. 1948). 
Jones, R. C., On the theory of fluctuations in the decay of sound, J. Acoust. Soc. Amer. 11, 324 (1940).

M 1, 118

-Kakutani, S. (See K. Yosida), Proc. Imp. Acad. Jap. 14, 333 (1938).

Z 20, 39

Kampé de Fériet, J., Sur le spectre de la turbulence homogène, C. R. Acad. Sci. Paris 208, 722 (1939).

Z 20, 313

Kampé de Fériet, J., Sur la moyenne des mesures, dans un écoulement turbulent, des anémomètres dont les indications sont indépendantes de la direction de la vitesse, La Météorologie 1, 133 (1946).

M 9, 47

Kampé de Fériet, J., Le tenseur spectral de la turbulence homogène non isotrope dans un fluide incompressible, C. R. Acad. Sci. Paris 22\%, 760 (1948).

M 10, 200

Kampé de Fériet, J., Introduction to the statistical theory of turbulence. Correlation and spectrum. (Inst. Dyn. \& App. Math., Univ. Md., College Park, 1951).

M 13, 398

Kampé de Fériet, J., Sur l'analyse spectrale d'une fonction stationnaire en moyenne, Colloque Inter. Mécanique, III, 317 (Ministère Air, Paris, 1951).

M 13, 477

Kampé de Fériet, J., L’intégration de l'équation de la chaleur pour des données initiales aléatoires, Mémoires, p. 153 (Publ. Sci. Tech. Ministère Air, Paris) (1954).

M 16, 268

Kendall, D. G., Stochastic processes and population growth, J. Roy. Statist. Soc. Ser. B. 11, 230 (1949).

M 11, 672

Kendall, D. G., Some problems in the theory of queues, J. Roy. Statist. Soc. Ser. B. 13, 151 (1951).

M 13,957

- Kendall, D. G. (See M. S. Bartlett), Proc. Cambridge Philos. Soc. 4\%, 65 (1951).

Kendall, D. G., Stochastic processes occurring in the theory of queues and their analysis by the method of the imbedded Markov chain, Ann. Math. Statist. 24, 338 (1953).

M 15, 44

Kern, E. R., The theory of risk and its use. Contributions to the study of stability and retention limits in insurance, Univ. Buenos Aires. Fac. Ci. Econ. 5, 7 (1952).

M 14, 887

Kitagawa, T., The weakly contagious discrete stochastic process, Mem. Fac. Sci. Kyūsyū Imp. Univ. A. 2,37 (1941).

M 3, 170

Kitagawa, T., The limit theorems of the stochastic contagious processes, Mem. Fac. Sci. Kyūsyū Imp. Univ. A. 1, 167 (1941).

M 2,230

Kitagawa, T., The weakly contagious stochastic process which depends upon the Gaussian distribution, Mem. Fac. Sci. Kyūsyū Imp. Univ. A. 2, 27 (1941).

M 3, 170

Klemenc, A. (See G. Heinrich), Acta Physica Austriaca 4, 160 (1950).

M 12, 788

Kosten, L., On the frequency distribution of the number of discharges counted by a Geiger-Müller counter in a constant interval, Physica 10, 749 (1943).

M 6, 161
Kovasznay, L. S. G. (See M. S. Uberoi), Quart. Appl. Math. 10, 375 (1953).

Koyenuma, N., Beiträge zur Theorie der biologischen Strahlenwirkung, Z. Phys. 120, 185 (1943).

M 4, 201

Kramers, H. A., Brownian motion in a field of force and the diffusion model of chemical reactions, Physica \%, 284 (1940).

M 2,140

Krĕ̌n, M. G., On a basic approximation problem of the theory of extrapolation and filtration of stationary random processes, Doklady Akad. Nauk SSSR 94, 13 (1954).

M 16, 53

Kronig, R., On time losses in machinery undergoing interruptions, II, Physica 10, 331 (1943).

M 6, 161

Kronig, R., On time losses in machinery undergoing interruptions, Physica 10, 215 (1943). M 6, 161

Krutkow, G., Zur Theorie der Brownschen Bewegung. Über die Verteilungsfunktion $\mathrm{f}(\mathrm{v}, \mathrm{x}, \mathrm{t})$ und die Diffusionsgleichung, C. R. Acad. Sci. URSS 1, 393 (1934).

Z 8, 399

Kunisawa, K., The mathematical foundation of Shannon's information source and its application to binary coding, Rep. Statist. Appl. Res. Union. Jap. Sci. Eng. 2, No. 1, 4 (1952).

M 14, 772

Landé, A., Transitions between levels spaced almost continuously, Phys. Rev., II. 54, 940 (1938).

$\mathrm{Z} \mathbf{2 0}, 44$

Laplume, J., Sur le nombre de signaux discernables en présence du bruit erratique dans un système de transmission à bande passante limitée, $C$. $R$. Acad. Sci. Paris 226, 1348 (1948). $\quad$ M 9, 599

- Lapostolle, P. (See Blanc-Lapierre), C. R. Acad. Sci. Paris 2\%2, 1324 (1946).

- Lapostolle, P. (See Blanc-Lapierre), J. Phys. Radium \%, 153 (1946).

-Leontovich, M. A. (See V. I. Bunimovich), C.R. Acad. Sci. URSS 53, 21 (1946).

Leontovič, M., Die Grundgleichungen der kinetischen Gastheorie vom Gesichtspunkt der Zufallsprozesse, Z. eksper. teoret. Fis. 5, 211 (1935).

Z 12, 268

Leslie, P. H., On the distribution in time of the births in successive generations, J. Roy. Statist. Soc. Ser. A. 111, 44 (1948). M 10, 552

- Levert, C., Probability fluctuations of discharges in a Geiger-Müller counter produced by cosmic radiation, Physica 10, 225 (1943). M 6, 160

Linnik, Yu. V., Application of the theory of Markov chains to the arithmetic of quaternions, Uspehi Mat. Nauk 9, 203 (1954).

M 16, 1002

Lotka, A. J., Application of recurrent series in renewal theory, Ann. Math. Statist. 19, 190 (1948).

M 10, 133

Lundberg, O., On Random Processes and their Application to Sickness and Accident Statistics, (Univ. Stockholm Thesis Uppsala, 1940). M 2,230

Lundberg, O., On the importance of regarding risk premiums in voluntary sickness and accident insurance. A theoretical basis for regrading, Trans. Int. Cong. Actuar., II, 543 (O. Füssli, Zurich, 1941). 
MacDonald, D. K. C., Some statistical properties of random noise, Proc. Cambridge Philos. Soc. 45, 368 (1949).

M 11, 118

MacDonald, D. K. C., Spontaneous fluctuations, Reports on Progress in Physics 12, 56 (1949).

M 12, 426

Malécot, G., Les processus stochastiques de la génétique, Colloq. Int. Centre Nat. Rech. Sci. 13, 121 Paris (1949).

M 11, 449

Malécot, G., Les processus stochastiques et la méthode des fonctions génératrices ou caractéristiques, Publ. Inst. Statist. Univ. Paris 1, No. 3, 1 (1952).

M 16, 56

Mandelbrot, B., Contribution à la théorie mathématique des jeux de communication, Publ. Inst. Statist. Univ. Paris 2, Nos. 1-2, (1953). M 15, 238

Massé, P., Sur les principes de la régulation d'un débit aléatoire par un réservoir, C.R. Acad. Sci. Paris 219, 19 (1944).

M $\boldsymbol{g}, 316$

Massé, P., Sur les effets de la régulation d'un débit aléatoire par un reservoir, C. R. Acad. Sci. Paris 219, 150 (1944).

$\mathrm{M} \boldsymbol{\gamma}, 316$

Massé, P., Sur un cas particulier remarquable de la régulation d'un débit aléatoire par un réservoir, C. R. Acad. Sci. Paris 219, 173 (1944). M \%, 316

- Mathews, P. M., (See A. Ramakrishnan), Philos. Mag. 44, 1122 (1953).

- Mathews, P. M., (See A. Ramakrishnan), Progress Theoret. Physics 11, 95 (1954).

Middleton, D., Spurious signals cauesd by noise in triggered circuits, J. Appl. Phys. 19, 817 (1948).

M 10, 311

४Miller, K. W., (See H. A. Adler), Trans, Amer. Inst. Elec. Engrs. 65, 630 (1946).

-Mondria, H., (See R. Kronig), Physica 10, 331 (1943).

-Moppert, K. F., (See F. Grün), Helvetica Phys. Acta 2\%, 417 (1954).

- Morse, P. M., A family of queuing problems, J. Operations Res. Soc. Am. 2, 444 (1954).

M 16, 379

$\checkmark$ Mosteller, F., (See R. R. Bush), Ann. Math. Statist. 24, 559 (1953).

Moyal, J. E., Stochastic processes and statistical physics, J. Roy. Statist. Soc. Ser. B. 11, 150 (1949).

M 11, 672

Moyal, J. E., The spectra of turbulence in a compressible fluid; eddy turbulence and random noise, Proc. Cambridge Philos. Soc. 48, 329 (1952).

M 14, 106

Nagabhushanam, K., The primary process of a smoothing relation, Ark. Mat. 1, 421 (1951).

M 12, 840

Nolfi, P., Zur mathematischen Darstellung wachsender Gesamtheiten, Mitt. Verein. Schweiz. Versich-Math 45, 311 (1945).

M \%, 311

O'Brien, G. G., The solution of some queueing problems J. Soc. Indust. Appl. Math. 2, 133 (1954).

M 16, 600
Obuhov, A. M., The statistical description of continuous fields, Trudy Geofiz. Inst. No. 24, 3 (1954).

M 16, 725

Opatowski, I., Chain processes and their biophysical applications. II. The effect of recovery, Bull. Math. Biophys. 8, 7 (1946).

М $\%, 319$

Opatowski, I., The probabilistic approach to the effects of radiations and variability of sensitivity, Bull. Math. Biophys. 8, 101 (1946).

M 8, 45

Osida, I., Statistical theory of transport phenomena, Proc. Phys.-Math. Soc. Japan 25, 590 (1943).

M \%, 315

Ottaviani, G., La teoria del rischio del Lundberg e il suo legame con la teoria classica del rischio, Giorn. 1st. Ital. Attuari 11, 163 (1940).

M 8, 390

Palm, C., Intensitätsschwankungen im Fernsprechverkehr, (Eriesson Technics No. 44, 1943). M 6, 160

Pitt, H. R., A theorem on random functions with applications to a theory of provisioning, $J$. London Math. Soc. 21, 16 (1946).

M 8, 281

Pollaczek, F., Sur l'application de la théorie des fonctions au calcul de certaines probabilités continues utilisées dans la théorie des réseaux téléphoniques, Ann. Inst. H. Poincaré 10, 1 (1946).

М 9, 362

Pollaczek, F., La loi d'attente des appels téléphoniques, C. R. Acad. Sci. Paris 222, 353 (1946).

M $\boldsymbol{\%}, 461$

Pollaczek, F., Sur un problème du calcul des probabilités qui se rapporte à la téléphonie, J. Math. Pures Appl. 25, 307 (1947).

M 9, 451

Pollaczek, F., Sur la théorie stochastique des compteurs électroniques, C. R. Acad. Sci. Paris 238, 766 (1954).

M 15, 542

Ramakrishnan, A., Stochastic processes relating to particles distributed in a continuous infinity of states, Proc. Cambridge Philos. Soc. 46, 595 (1950).

M 14, 296

Ramakrishnan, A., A note on Jánossy's mathematical model of a nucleon cascade, Proc. Cambridge Philos. Soc. 48, 451 (1952).

M 14, 388

-Ramakrishnan, A., A stochastic problem relating to counters, Philos. Mag. 44, 1122 (1953).

M 15, 723

- Ramakrishnan, A., Studies on the stochastic problem of electron-photon cascades, Progress Theoret. Physics 11, 95 (1954).

M 15, 807

$\checkmark$ Ramakrishnan, A., Two simple stochastic models of cascade multiplication, Progress Theoret. Physics 11, 595 (1954).

M 16, 148

Ramakrishnan, A., Counters with random dead time, Philos. Mag. 45, 1050 (1954).

M 16, 149

Reid, A. T., On stochastic processes in biology, Biometrics 9, 275 (1953).

M 15, 242

Rice, S. O., Filtered thermal noise-fluctuation of energy as a function of interval length, J. Acoust. Soc. Am. 14, 216 (1943).

M 4, 205

Rice, S. O., Mathematical analysis of random noise, Bell System Tech. J. 23, 282 (1944).

Rice S. O., Mathematical analysis of random noise, Bell System Tech, J. 24, 46 (1945). M 6, 89 
Rice, S. O., Communication in the presence of noiseprobability of error for two encoding schemes, Bell System Tech. J. 99, 60 (1950).

M 11, 445

-Rosenblatt, M., (See U. Grenander) Trans. Am.

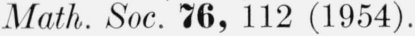

Saxén, T., On the probability of ruin in the collective risk theory for insurance enterprises with only negative risk sums, Skand, Aktuarietidskr. 31, 199 (1948).

M 10, 464

Saxén, T., Sur les mouvements aléatoires et le problème de ruine de la théorie de risque collective, Soc. Sci. Fenn. Comment. Phys.-Math. 16, No. 2, 55 pp. (1951).

M 14, 1100

- Scheen, W. L., (See C. Levert) Physica 10, 225 (1943).

Schulz, G., Zur Theorie des Galtonschen Brettes, Z. Physik 92, 747 (1934).

Z 10, 214

Segerdahl, C. O., Über einige risikotheoretische Fragestellungen, Skand. Aktuarietidskr. 25, 43 (1942).

M 8, 215

Segerdahl, C. O., Some properties of the ruin function in the collective theory of risk, Skand. Aktuarietidskr. 31, 46 (1948).

M 10, 463

Shannon, C. E., A mathematical theory of communication, Bell System Tech. J. 97, 379 (1948).

M 10, 133

- Shannon, C. E., The Mathematical Theory of Communication (Univ. Illinois Press, Urbana, 1949).

M 11, 258

Shannon, C. E., Communication theory of secrecy systems, Bell System Tech. J. 28, 656 (1949).

Mi 11, 258

Simonsen, W., On the foundation of the collective risk theory (Studier T. F. Lundberg p. 246, Stockholm, 1946).

M 8, 391

- Srinivasan, S. K. (See A. Ramakrishnan), Progress Theoret. Physics 11, 595 (1954).

Takács, L., Wahrscheinlichkeitstheoretische Behandlung von Koinzidenz-Erscheinungen, mit Ereignissen gleicher Zeitdauer, Comptes Rendus du Premier Cong. Math., Sept. 1950, p. 731 (Akad. Kiadó, Budapest, 1952).

M 14, 1101

Takács, L., Coincidence problems arising in the theory of counters, Magyar Tud. Akad. Alkalm. Mat. Int. Közl. 2, 153 (1954).

M 16, 379

Takács, L., "Waiting time" problems treated by means of Markov processes, Magyar Tud. Akad. Mat. Fiz. Oszt. Közl. 4, 543 (1954). M 16, 723

-Turán, P. (See E. Egerváry), Studia Math. 12, 170 (1951). $\checkmark$ Uberoi, M. S., On mapping and measurement of random fields, Quart. Appl. Math. 10, 375 (1953).

M 14, 486

-Ullman, R. (See J. J. Hermans), Physica 18, 951 (1952).

Vaulot, E., Délais d'attente des appels téléphoniques traités au hasard, C.R. Acad. Sci. Paris 222, 268 (1946).

M $\boldsymbol{\gamma}, 461$

Ville, J. A., Fonctions aléatoires et transmission de l'information, Colloq. Inter. Centre Nat. Rech. Sci. Paris, 13, 115 (1949).

M 11, 259

Weaver, W. (See C. E. Shannon) (Univ. Illinois Press, Urbana, 1949).

-Wehrlé, P. (See G. Dedebant), Thalès 4, 151 (1940).

-Wehrlé, P. (See G. Dedebant), Portugaliae Phys. 1, 179 (1945).

-Wehrlé, P. (See J. Bass), C. R. Acad. Sci. Paris 220, 165 (1945).

Wiener, N., Extrapolation, Interpolation, and Smoothing of Stationary Time Series. With Engineering Applications (John Wiley \& Sons, New York, 1949)

M 11, 118

Wiener, N., Time, communication, and the nervous system, Ann. New York Acad. Sci. 50, 197 (1948).

M 10, 133

Wiener, N., Cybernetics, or Control and Communication in the Animal and the Machine (John Wiley \& Sons, Inc., New York, 1948).

M 9, 598

Wisniewski, F. J., Les probabilités à structure héréditaire et la statistique moléculaire, $A n n$. Mat. Pura Appl., IV. 12, 33 (1933). Z Z g, 315

Wold, H., On the inversion of moving averages, Skand. Aktuarie Tidskr. 21, 208 (1938).

Z 20, 147

Wold, H. (See R. Bentzel), Skand. Aktuarietidskr. 29, 95 (1946).

Yaglom, A. M., On the statistical treatment of Brownian motion, Doklady Akad. Nauk SSSR 56, 691 (1947).

M 9, 150

Yosida, K., Application of mean ergodic theorem to the problems of Markoff's process, Proc. Imp. Akad. Jap. 14, 333 (1938).

$\mathrm{Z} \mathbf{2 0}, 39$

Yuškevič, A. A., On limit theorems connected with the concept of entropy of Markov chains, Uspehi Matem. Nauk 8, 177 (1953).

(Paper 65B1-46) 



\title{
Publications of the National Bureau of Standards*
}

\author{
(Including Papers in Outside Journals)
}

\section{Selected Abstracts}

\begin{abstract}
Statistical models for component aging experiments, J. R. Rosenblatt, Intern. Conv. Record. Inst. Radio Engrs. 8, Pt. $6,115(1960)$.

Statistical methods are considered for analysis of experiments in which electronic components (transistors, say) are subjected to controlled "aging" conditions and measured periodically until "failure". A general framework is proposed for probabilistic models representing the results of such experiments. Some techniques for statistical analysis are outlined which permit simultaneous treatment of variables data and "failure" data, and lead to some possible flexibility in the definition of "failure". The statistical techniques are directed to the problem of estimating the probability of the joint event that (a) the component survives to age $T$ and (b) the value of a component characteristic remains within specified limits.
\end{abstract}

Mechanized conversion of colorimetric data to Munsell renotations, W. Reinboldt and J. P. Menard, J. Opt. Soc. Am. 50, No. 8, 802 (Aug. 1960)

A program for a high-speed digital electronic computer is described for performing the computation of the Munsell renotations $H, V, C$ corresponding to given CIE chromaticity coordinates $x, y$ and daylight reflectance $Y$. Mathematically, this is equivalent to a three-dimensional coordinate transformation where two of the three transformation functions are given only numerically for a grid of discrete points. Since this grid consists of approximately 5000 points which are nonuniformly spaced, the major problem was to devise an economic scanning routine in order to find the point used in the interpolation. This was accomplished by consistent use of vector algebra and the help of an interpretive routine for vector operations.

Electric current and fluid spin created by the passage of a magnetosonic wave, R. P. Kanawal and C. Truesdell, Arch. Rational Mech. and Analysis, 5, No. 5, 432 (1960).

A general exact theory of weak discontinuities in ionized gases is constructed. Emphasis is put upon the connection between the electric current and fluid vorticity carried by the wave.

\section{List of Titles}

Journal of Research, Section 65A, No. 1, January-February 1961. 70 cents.

Faint lines in the arc spectrum of iron (Fe I). C. C. Kiess, V. C. Rubin, and C. E. Moore.

Infrared adsorption of spectra of some 1-acetamido pyranoid derivatives and reducing, acetylated pyranoses. R. S. Tipson and H. S. Isbell.

Monolayers of linear saturated succinate polyesters and airliquid interfaces. W. M. Lee, J. L. Shereshefsky, and R. R. Stromberg.

Heat of formation of beryllium chloride. W. H. Johnson and A. A. Gilliland.

Heat of decomposition of potassium perchlorate. W. H. Johnson and A. A. Gilliland.

Heats of formation of lithium perchlorate, ammonium perchlorate, and sodium perchlorate. A. A. Gilliland and W. H. Johnson.

Heat of formation of $N$-dimethylaminodiborane. W. $\mathrm{H}$. Johnson, I. Jaffe, and E. J. Prosen.

Separation of hafnium from zirconium by anion exchange. J. L. Hague and L. A. Machlan.

Reaction of sulfur, hydrogen sulfide, and accelerators with propylene and butadiene. F. J. Linnig, E. J. Parks, and L. A. Wall.

\section{Journal of Research, Section 65C, No. 1, January- March 1961. 75 cents.}

Electronic scanning microscope for a spectrographic plate comparator. M. L. Kuder.

Viscoelastometer for measurement of flow and elastic recovery. R. J. Overberg and H. Leaderman.

An ultra low frequency bridge for dielectric measurements. D. J. Scheiber.

The ephi system for VLF direction-finding. G. Hefley, R. F. Linfield, and T. L. Davis.

Fast counting of alpha particles in air ionization chambers. Z. Bay, F. D. MeLernon, and P. A. Newman.

$\mathrm{X}$-ray diffraction measurement of intragranular misorientation in alpha brass subjected to reverse plastic strain. C. J. Newton and H. C. Vacher.

Enthalpy and specific heat of nine corrosion-resistant alloys at high temperatures. T. B. Douglas and A. C. Victor.

Determination of minor constituents in low-alloy steels by X-ray fluorescence. R. E. Michaelis, R. Alvarez, and B. A. Kilday.

\section{Journal of Research, Section 65D, No. 1, January- February 1961. 70 cents.}

Incoherent scattering by free electrons as a technique for studying the ionosphere and exosphere: some observations and theoretical considerations. K. L. Bowles.

Radio wave absorption of several gasses in the 100 to 117 $\mathrm{kMc} / \mathrm{s}$ frequency range. C. O. Britt, C. W. Tolbert, and A. W. Straiton.

On the theory of diffraction by a composite cylinder. R. D. Kodis.

An atlas of oblique-incidence ionograms (a digest). V. Agy, K. Davies, and R. Salaman.

A new approach to the mode theory of VLF propagation J. R. Wait.

East-west effect on VLF mode transmission across the earth's magnetic field. D. Dobrott and A. Ishimaru.

Magneto-ionic propagation phenomena in low- and very-lowradiofrequency waves reflected by the ionosphere. J. R. Johler.

Correlation of monthly median transmission loss and refractive index profile characteristics. B. R. Bean and B. A. Cahoon.

Characteristics of waveguides for long-distance transmission. A. E. Karbowiak and L. Solymar.

Useful radiation from an underground antenna. H. A. Wheeler.

Observation of $F$-layer and sporadic- $E$ scatter at VHF in the Far East. K. Miya, T. Sasaki, and M. Ishikawa.

A high-resolution rapid-scan antenna. H. V. Cottony and A. C. Wilson.

Alphabetical index to tables of chemical kinetics. Homogeneous reactions. Supplement 2 to NBS Circ. 510 (1960) 35 cents.

The metric system of measurement, NBS Misc. Publ. 232 (1960) 50 cents.

Standard X-ray diffraction powder patterns, H. E. Swanson, M. I. Cook, E. H. Evans, and J. H. deGroot. NBS Circ. 539, Vol. 10 (1960) 40 cents.

Stabilization of free radicals at low temperatures, edited by A. M. Bass and H. P. Broida. NBS Mono. 12 (1960) \$1.50.

Heat treatment and properties of iron and steel, T. G. Digges and S. J. Rosenberg. NBS Mono. 18 (1960) 35 cents. (Supersedes C495). 
Specific heats and enthalpies of technical solids at low temperatures. A compilation from the literature, R. J. Corruccini and J. J. Gniewek. NBS Mono. 21 (1960) 20 cents.

Standardization activities in the United States. A descriptive directory, S. F. Booth. NBS Misc. Publ. 230 (1960) (Supersedes M169) \$1.75.

Hydraulic Research in the United States, 1960, H. K. Middleton. NBS Misc. Publ. 231 (1960) \$1.00.

Household weights and measures. NBS Misc. Publ. 234 (1960) (Supersedes M39) 5 cents.

Screw-thread standards for federal services, 1957. Amends in part H28 (1944) (and in parts its 1950 Supplement). NBS Handb. H28 (1957)-Part III (1960) 60 cents.

Protection against radiations from sealed gamma sources. NBS Handb. 73 (1960) (Supersedes H54) 30 cents.

Quarterly radio noise data-June, July, August 1959, W. Q. Crichlow, R. D. Disney, and M. A. Jenkins. NBS TN18-3 (PB151377-3) (1960) \$1.00.

Investigation of bearing creep of two forged aluminum alloys, L. Mordfin, N. Halsey, P. J. Granum. NBS TN55 (PB161556) (1960) \$1.00.

A survey and bibliography of recent research in the propagation of VLF radio waves, J. R. Wait. NBS TN58 (PB161559) (1960) 75 cents.

Amplitude and phase of the low- and very low-radiofrequency ground wave, J. R. Johler, L. C. Walters, and C. N. Lilley. NBS TN60 (PB161561) (1960) 75 cents.

Rapid determination of the order of chemical reactions from time-ratio tables, J. H. Flynn. NBS TN62 (PB161563) (1960) 75 cents.

Single scattered neutrons from an isotropic point source, E. R. Mosburg, Jr., and W. M. Murphey. NBS TN63 (PB161564) (1960) 50 cents.

Radio refractometry, J. W. Herbstreit. NBS TN66 (PB161567) (1960) 50 cents.

Transistorized building blocks for data instrumentation, J. A Cunningham and R. L. Hill. NBS TN68 (PB161569) (1960) $\$ 2.00$.

Low- and very low-radiofrequency model ionosphere reflection coefficients, J. R. Joyler, L. C. Walters, J. D. Harper, Jr. NBS TN69 (PB161570) (1960) \$2.00.

Calibration of five gamma-emitting nuclides for emission rate, J. M. R. Hutchinson. NBS TN71 (PB161572) (1960) 75 cents.

Table of magnitude of reflection coefficient versus return loss $\left(L_{R}=20 \log _{10} \frac{1}{|\Gamma|}\right)$ R. W. Beatty and W. J. Anson. NBS TN72 (PB161573) (1960) \$1.25.

$\mathrm{VHF}$ and UHF power generators for RF instrumentation, A. H. Morgan and P. A. Hudson. NBS TN77 (PB161578) (1960) 75 cents.

Clay mineral content of two domestic kaolins, W. C. Ormsby and J. M. Shartsis, J. Am. Ceram. Soc. 43, No. 6, 335 (June 1960).

Some canons of sound experimentation, C. Eisenhart, Bull. Inst. Intern. Stat. XXXVII, No. 3, 339 (1960).

Thermodynamic properties of helium at low temperatures and high pressures, D. B. Mann and R. B. Stewart, Trans. Am. Soc. Mech. Engrs. J. Heat Transfer 81, No. 4, 324 (Nov. 1959).

Interpolation of platinum resistance thermometers, $20^{\circ}$ to $273.15^{\circ}$ K, R. J. Corruccini, Rev. Sci. Instr. 31, No. 6, 637 (June 1960).

Radiation from a slot on a large corrugated cylinder, J. R. Wait and A. M. Conda, Electromagnetic Wave Propagation, Proc. Intern. Conf. Propagation of Radio Waves, Liege, Belgium, 1958, p. 103 (Academic Press, Inc., New York, N.Y., 1960).

Low-temperature transport properties of commercial metals and alloys. IV. Reactor grade Be, Mo, and W, R. L. Powell, J. L. Harding, and E. F. Gibson, J. Appl. Phys. 31, No. 7, 1221 (July 1960).

A rating scale method for evaluating research positions, H. A. McKean, J. Mandel, and M. N. Steel, Personnel Admin. 23, No. 4, 29 (July-Aug. 1960).

Post office mechanization, B. M. Levin, M. C. Stark, and P. C. Tosini, Proc. Information Processing and Retrieval, Am. Inst. Elec. Engrs. General Meeting, June 19-24, 1960,
Atlantic City, New Jersey, Am. Inst. Elec. Engrs. Paper CP60-937 (1960).

Sensitive thermal conductivity gas analyzer, J. R. Purcell and R. N. Keeler, Rev. Sci. Instr. 31, No. 3, 304 (Mar. 1960).

A study of two water resistance testers for shoe upper leather, R. L. Hebert, J. Am. Leather Chemists Assoc. LV, No. 7, 388 (July 1960).

Transfer of liquid hydrogen through uninsulated lines, R. J. Richards, W. G. Steward, and R. B. Jacobs, Proc. 1959 Cryogenic Engr. Conf., Vol. 5, Advances in Cryogenic Engineering, p. 103 (Plenum Press, New York, N.Y., 1960).

Bounds on the dissipation of energy in steady flow of a viscous incompressible fluid around a body rotating within a finite region, E. A. Kearsley, Arch. Rational Mech. and Analysis 5, No. 4, 347 (1960)

Low and medium frequency radio propagation, K. A. Norton, Electromagnetic Wave Propagation, Proc. Intern, Conf. Propagation of Radio Waves, Liege, Belgium, 1958, p. 375 (Academic Press, Inc., New York, N.Y., 1960).

Cavity resonator dielectric measurements of rod samples, H. E. Bussey, Conf. Electrical Insulation (Annual Report of 1959), Natl. Acad. Sci., Natl. Research Council Pub. 756 , $15(1960)$

Analysis of fractionally replicated $2^{m} 3^{n}$ designs, R. C. Bose and W. S. Connor, Bull. Inst. Intern. Stat. XXX II, No. 3, 141 (1960).

Halobenzenes as sensitizers for the radiation-induced polymerization of styrene, D. W. Brown and L. A. Wall, J. Polymer Sci. 44, 325 (June 1960).

Some aspects of fluorine flame spectroscopy, D. E. Mann. Proc. Propellant Thermodynamics and Handling Conf. Special Rept. 12 (Ohio State University, Columbus, Ohio, June 1960).

Optical constants of aluminum, H. Mendlowitz. Proc. Phys. Soc. (London, England) LXXV, 664-670 (1960).

Current thermodynamic research on light-element compounds at the National Bureau of Standards, T. B. Douglas. Proc. Propellant Thermodynamics and Handling Conf. Special Rept. 12 (Ohio State University, Columbus, Ohio, June 1960).

Free radicals in gamma-irradiated polystyrenes, R. E. Florin, L. A. Wall, and D. W. Brown, Trans. Faraday Soc. 56, No. 453, 1304 (Sept. 1960).

Sealed-off $\mathrm{Hg}^{198}$ atomic-beam light source, R. L. Barger and K. G. Kessler. J. Opt. Soc. Am. 50, No. 7, 651 (July 1960).

Temperature dependence of Young's modulus of vitreous germania and silica, S. Spinner and G. W. Cleek. J. Appl. Phys. 31, No. 8, 1407 (1960).

Atomic clocks for space experiments, P. L. Bender, Astronautics p. 69 (July 1960).

Photolysis of ammonia in a solid matrix at low temperatures, O. Schnepp and K. Dressler. J. Chem. Phys. 32, No. 6, 1682 (June 1960).

Photochemical rates in the equatorial $\mathrm{F}_{2}$ region from the 1958 eclipse, T. E. Van Zandt, R. B. Norton, and G. H. Stonehocker, J. Geophys. Research 65, No. 7, 2003 (July 1960).

Influence of source distances on the impedance characteristics of ELF radio waves, J. R. Wait, Proc. IRE 48, No. 7, 1338 (July 1960).

Electroless plated contacts to silicon carbide, R. L. Raybold. Rev. Sci. Instr. 31, No. 7, 781 (July 1960).

Isotope effect in the hydrogen atom-formaldehyde reaction, J. R. McNesby, M. D. Scheer, and R. Klein, J. Chem. Phys. 32, No. 6, 1814 (June 1960).

The nature of the inorganic phase in calcified tissues, A. S. Posner. Calcification in Biological Systems, p. 373 (American Assoc. Advancement of Sci., Washington, D.C., 1960).

Effect of water-reducing admixtures and set-retarding admixtures on properties of concrete, Introduction and Summary, B. E. Foster. Am. Soc. Testing Materials Spec. Tech. Publ. 266, Introduction 1 \& 2 and Summary 240 (June 1960).

Absorption spectra of solid methane, ammonia, and ice in the vacuum ultraviolet, K. Dressler and O. Schnepp, J. Chem. Phys. 33, No. 1, 270 (July 1960).

The extent of 'H II regions, S. R. Pottasch, Astrophys. J. 132, No. 1, 269 (July 1960). 
Nickel oxide thin film resistors for low pressure shock wave detection, K. E. McCullon. Rev. Sci. Instr. 31, No. 7, 780 (July 1960).

Casimir coefficients and minimum entropy production, R. E. Nettleton, J. Chem. Phys. 33, No. 1, 237 (July 1960).

Variations of surface tension calculated with improved approximation for activity coefficient, L. C. Shepley and A. B. Bestul. J. Am. Ceram. Soc. 43, No. 7, 386 (July 1960).

Council adopts F. D. I. specification for alloy for dental amalgam, Council on Dental Research, J. Am. Dental Assoc. 60, No. 6, 773 (June 1960).

The foundations of mechanics and thermodynamics, E. A. Kearsley and M. S. Green, Phys. Today 13, No. 7, 22 July 1960).

Optical methods for negative ion studies, S. J. Smith and L. M. Branscomb, Rev. Sci. Instr. 31, No. 7, 733 (July 1960).

On the theory of the slow-tail portion of atmospheric waveforms, J. R. Wait, J. Geophys. Research 65, No. 7, 1939 (July 1960).

Statistical aspects of the cement testing program, W. J. Youden. Am. Soc. Testing Materials Proc. 59, 1120 (1959).

Optical transmissivity and characteristic energy losses, $H$. Mendlowitz, J. Opt. Soc. Am. 50, No. 7, 739 (July 1960).

Pyrolysis of polyolefins, L. A. Wall and S. Straus, J. Polymer Sci. 44, 313 (June 1960).

A barium fluoride film hygrometer element, F. E. Jones, and A. Wexler, J. Geophys. Research 65, No. 7, 2087 (July 1960).

Low-energy photoproduction of neutral mesons from complex nuclei, R. A. Schrack, S. Penner, and J. E. Leiss, II Nuovo Cimento 16, Serie X, 759 (Mar. 1960).

Adsorption spectra of solid xenon, krypton, and argon in the vacuum ultraviolet, O. Schnepp and K. Dressler, J. Chem. Phys. 33, No. 1, 49 (July 1960).

Radiation patterns of finite-size corner-reflector antennas, A. C. Wilson, H. V. Cottony, IRE Trans. Ant. Prop. AP-8, No. 2, 144 (Mar. 1960).

Structure of sulfurous esters, H. Finegold, Proc. Chem. Soc. (London) 283 (Aug. 1960).

VLF attenuation for east-west and west-east daytime propagation using atmospheries, W. L. Taylor, J. Geophys. Research 65, No. 7, 1933 (July 1960).

The use of geostationary satellites for the study of ionospheric electron content and ionospheric radio-wave propagation, O. K. Garriott and C. G. Little, J. Geophys. Research 65, No. 7, 2025 (July 1960).

Our measurement system and national needs, A. V. Astin, Sperryscope 15, No. 6, 16 (1960).

Standards of heat capacity and thermal conductivity, D. C. Ginnings, Book, Thermoelectricity (Including Proc. Conf. Thermoelectricity, Sponsored by the Naval Research Lab., September 1958), p. 320 (1960).

Neutron-insensitive proportional counter for gamma-ray dosimetry, R. S. Caswell, Rev. Sci. Instr. 31, No. 8, 869 (Aug. 1960).

A study of $2-\mathrm{Mc} / \mathrm{s}$ ionospheric absorption measurements at high altitudes, K. Davies, J. Geophys. Research 65, 2285 (Aug. 1960).

Carrier-frequency dependence of the basic transmission loss in tropospheric forward scatter propagation, K. A. Norton, J. Geophys. Research 65, 2029 (July 1960).

The ionization constants of 2-chloro-4-nitrophenol and 2-nitro-4-chlorophenol, V. E. Bower and R. A. Robinson, J. Phys. Chem. 64, 1078 (1960).

Closed circuit liquid hydrogen refrigeration system, D. B. Chelton, J. W. Dean, and B. W. Birmingham, Rev. Sci. Instr. 31, 712 (July 1960).
A quantitative formulation of Sylvester's law of inertia, A. M. Ostrowski, Natl. Acad. Sci. Proc. 45, No. 5, 740 (May 1959).

Infrared transmission of clouds, D. M. Gates and C. C. Shaw, J. Opt. Soc. Am. 50, 876 (Sept. 1960).

Comparative fixation of calcium and strontium by synthetic hydroxyapatite, R. C. Likins, H. G. McCann, A. S. Posner, and D. B. Scott, J. Biolog. Chem. 235, No. 7, 2152 (July 1960).

A comparison of atomic beam frequency standards, R. E. Beehler, R. C. Mockler, and C. S. Snider, Nature Letter 187, 681 (Aug. 1960).

VI. Microscopic and macroscopic energy loss distributions. 1. Theoretical reviews: A summary, U. Fano, Natl. Acad. Sci., Natl. Research Council Pubj.' $\mathbf{7 5 2}$, Report 29, p. 24 (Aug.1960).

The mechanical properties of ceramics and their measurement at elevated temperatures, S. J. Schneider, Book, Thermoelectricity, Chapter 21, 342 (1960).

A rating method for refrigerated trailer bodies hauling perishable foods, C. W. Phillips, W. F. Goddard, Jr., and P. R. Achenbach, ASHRAE J. 2, No. 5, 45 (May 1960).

Reply to, On the structure of trimethylamine-trimethylboron, D. R. Lide, Jr., J. Chem. Phys. 32, No. 5, 1570 (May 1960). Dimensional changes occurring in dentures during processing, J. B. Woelfel, G. C. Paffenbarger, and W. T. Sweeney, J. Am. Dental Assoc. 61, No. 4, 431 (Oct. 1960).

Teeth, artificial, G. C. Paffenbarger and G. B. Denton, Encyclopaedia Britannica 21, 878 (Jan. 1960).

A method of improving isolation in multi-channel waveguide systems, G. F. Engen, IRE Trans. Microwave Theory and Tech. Letter MTT-8, 460 (July 1960).

Influence of earth curvature and the terrestrial magnetic field on VLF propagation, J. R. Wait and K. Spies, J. Geophys. Research 65, 2325 (Aug. 1960).

Charge transfer and electron production in $\mathrm{H}^{-}+\mathrm{H}$ collisions, D. G. Hummer, R. F. Stebbings, W. L. Fite and L. M. Branscomb, Phys. Rev. 2, 668 (July 1960).

The characteristic energy losses of electrons in carbon, L. B. Leder and J. A. Suddeth, J. Appl. Phys. 8, 1422 (Aug. 1960).

Note historique sur les premieres annees de la microscopie electronique, L. Marton, Extrait Bull. Acad. Roy. Belg. (Classe des Sciences) 5, 119 (Mar. 1959).

An optical study of the boundary layer transition processes in a supersonic air system, W. Spangenberg and W. R. Rowland, Phys. of Dluids 3, No. 5, 667 (Sept.-Oct. 1960).

Thermodynamic structure of the outer solar atmosphere. VI. Effect of departures from the Saha equation on inferred properties of low chromosphere, S. R. Pottasch and R. N. Thomas, Astrophys. J. 132, 195 (July 1960).

Polymer decomposition: Thermodynamics, mechanisms, and energetics, L. A. Wall, Soc. Plastic engrs. Pt. I, 810-814 (Aug. 1960); Pt. II, 1031 (Sept. 1960).

The role of surface tension in determining certain clay-water properties, W. C. Ormsby, Bull. Am. Ceramic Soc. 39, No. 8, 408 (Aug. 1960).

* Publications for which a price is indicated (except for Technical Notes) are available only from the Superintendent of Documents, U.S. Government Printing Office, Washington 25, D.C. (foreign postage, one-fourth additional). Technical Notes are available only from the Office of Technical Services, U.S. Department of Commerce, Washington 25, D.C. (Order by PB number.) Reprints from outside journals and the NBS Journal of Research may often be obtained directly from the authors. 